\title{
Infinite horizon risk-sensitive control of diffusions without any blanket stability assumptions
}

\author{
Ari Arapostathis ${ }^{\mathrm{a}}$, Anup Biswas ${ }^{\mathrm{b}}$ \\ ${ }^{a}$ Department of Electrical and Computer Engineering, The University of Texas at Austin, \\ 2501 Speedway St., EER 7.824, Austin, TX 78712 \\ ${ }^{b}$ Department of Mathematics, Indian Institute of Science Education and Research, \\ Dr. Homi Bhabha Road, Pune 411008, India
}

\begin{abstract}
We consider the infinite horizon risk-sensitive problem for nondegenerate diffusions with a compact action space, and controlled through the drift. We only impose a structural assumption on the running cost function, namely near-monotonicity, and show that there always exists a solution to the risk-sensitive Hamilton-Jacobi-Bellman (HJB) equation, and that any minimizer in the Hamiltonian is optimal in the class of stationary Markov controls. Under the additional hypothesis that the coefficients of the diffusion are bounded, and satisfy a condition that limits (even though it still allows) transient behavior, we show that any minimizer in the Hamiltonian is optimal in the class of all admissible controls. In addition, we present a sufficient condition, under which the solution of the HJB is unique (up to a multiplicative constant), and establish the usual verification result. We also present some new results concerning the multiplicative Poisson equation for elliptic operators in $\mathbb{R}^{d}$.
\end{abstract}

Keywords: Risk-sensitive control, multiplicative Poisson equation, controlled diffusions, nonlinear eigenvalue problems, Hamilton-Jacobi-Bellman equation, monotonicity of principal eigenvalue 2010 MSC: Primary: 35R60, 93E20, Secondary:

\section{Introduction}

Optimal control under a risk-sensitive criterion has been an active area of research for the past 30 years. It has found applications in finance [9, 27, 43], missile guidance [47], cognitive neuroscience [44], and many more. There are many situations which dictate the use of a risk-sensitive penalty. For example, if one considers the risk parameter to be small then it approximates the standard mean-variance type cost structure. Another reason that the risk-sensitive criterion is often desirable is that it captures the effects of higher order moments of the running cost in addition to its expectation. To the best of our knowledge, the risk-sensitive criterion was first considered in [32]. We also refer the reader to [49, 50] for an early account of risk-sensitive optimal controls. For discrete state space controlled Markov chains, the risk-sensitive optimal control problem is studied in [15 18, 20 22, 34, 48]. For optimal control problems where the dynamics are modeled by controlled diffusions, we refer the reader to [4 6, 10 12, 14, 24 26, 35, 41, 42].

In this paper we deal with nondegenerate diffusions, controlled through the drift, with the control taking values in a compact metric space (see (1.1)). The goal is to minimize an infinite horizon average risk-sensitive penalty, where the running cost is assumed to satisfy a near-monotonicity hypothesis (Definition 1.1). We study the associated Hamilton-Jacobi-Bellman (HJB) equation and characterize the class of optimal stationary Markov controls. In [26] a similar control problem is studied under the assumption of asymptotic flatness, and existence of a unique solution to the HJB is established. This work is generalized in [42], where

Email addresses: ari@ece.utexas.edu (Ari Arapostathis), anup@iiserpune.ac.in (Anup Biswas) 
the authors impose some structural assumptions on the drift and cost (e.g., the cost necessarily grows to infinity, the action set is a Euclidean space, etc). Risk-sensitive control problems with periodic coefficients are studied in [41]. Risk-sensitive control for a general class of controlled diffusions is considered in [10 12], under the assumption that all stationary Markov controls are stable. However, the studies in [10 12] neither establish uniqueness of the solution to the HJB, nor do they fully characterize the optimal stationary Markov controls. One of our main contributions in this article is the development of a basic theory that parallels existing results for the ergodic control problem.

The dynamics are modeled by a controlled diffusion process $X=\left\{X_{t}, t \geq 0\right\}$ which takes values in the $d$-dimensional Euclidean space $\mathbb{R}^{d}$, and is governed by the Itô stochastic differential equation

$$
\mathrm{d} X_{t}=b\left(X_{t}, U_{t}\right) \mathrm{d} t+\sigma\left(X_{t}\right) \mathrm{d} W_{t}
$$

All random processes in (1.1) live in a complete probability space $(\Omega, \mathfrak{F}, \mathbb{P})$. The process $W$ is a $d$-dimensional standard Wiener process independent of the initial condition $X_{0}$. The control process $U$ takes values in a compact, metrizable set $\mathbb{U}$, and $U_{t}(\omega)$ is jointly measurable in $(t, \omega) \in[0, \infty) \times \Omega$. The set $\mathfrak{U}$ of admissible controls consists of the control processes $U$ that are non-anticipative: for $s<t, W_{t}-W_{s}$ is independent of

$$
\mathfrak{F}_{s}:=\text { the completion of } \sigma\left\{X_{0}, U_{r}, W_{r}, r \leq s\right\} \text { relative to }(\mathfrak{F}, \mathbb{P}) \text {. }
$$

We impose the standard assumptions on the drift $b$ and the diffusion matrix $\sigma$ to guarantee existence and uniqueness of solutions. For more details on the model see Section 1.2 .

Let $c: \mathbb{R}^{d} \times \mathbb{U} \rightarrow[1, \infty)$ be continuous, and locally Lipschitz in its first argument uniformly with respect to the second. For $U \in \mathfrak{U}$ we define the risk-sensitive penalty by

$$
\Lambda_{x}^{U}=\Lambda_{x}^{U}(c):=\limsup _{T \rightarrow \infty} \frac{1}{T} \log \mathbb{E}_{x}^{U}\left[\mathrm{e}^{\int_{0}^{T} c\left(X_{t}, U_{t}\right) \mathrm{d} t}\right]
$$

and the risk-sensitive optimal values by

$$
\begin{aligned}
\Lambda_{x}^{*} & :=\inf _{U \in \mathfrak{U}} \Lambda_{x}^{U}, & \Lambda^{*} & :=\inf _{x \in \mathbb{R}^{d}} \Lambda_{x}^{*}, \\
\Lambda_{\mathrm{m}, x}^{*} & :=\inf _{U \in \mathfrak{U}_{\mathrm{SM}}} \Lambda_{x}^{U}, & \Lambda_{\mathrm{m}}^{*} & :=\inf _{x \in \mathbb{R}^{d}} \Lambda_{\mathrm{m}, x}^{*},
\end{aligned}
$$

where $\mathfrak{U}_{\mathrm{SM}}$ is the class of stationary Markov controls. For $v \in \mathfrak{U}_{\mathrm{SM}}$ we also let $\Lambda^{v}=\Lambda^{v}(c)=\inf _{x \in \mathbb{R}^{d}} \Lambda_{x}^{v}(c)$. A stationary Markov control $v$ which satisfies $\Lambda^{v}<\infty$, is called stabilizing, and we let $\mathfrak{U}_{\text {stab }}$ denote this class of controls.

Unless $\Lambda^{*}$ is finite, the optimal control problem, is of course ill-posed. For nonlinear models as in the current paper, standard Foster-Lyapunov conditions are usually imposed to guarantee that $\Lambda^{*}<\infty$. However, the objective of this paper is different. Rather, we impose a structural assumption on the running cost function $c$, and investigate whether this is sufficient for characterization of optimality via the risksensitive HJB equation. We need the following definition.

Definition 1.1 (Near-Monotone). A continuous map $g: \mathbb{R}^{d} \times \mathbb{U} \rightarrow \mathbb{R}$ is said to be near-monotone relative to $\lambda \in \mathbb{R}$ if there exists $\epsilon>0$ such that the set

$$
\mathcal{K}_{\epsilon}:=\left\{x \in \mathbb{R}^{d}: \min _{u \in \mathbb{U}} g(x, u) \leq \lambda+\epsilon\right\}
$$

is compact (or empty). We extend the same notion to a Borel measurable $f: \mathcal{X} \rightarrow \mathbb{R}$, by requiring that for some $\epsilon>0$, and a compact set $\mathcal{K}_{\epsilon} \subset \mathbb{R}^{d}$ it holds that ess $\inf _{\mathcal{K}_{\epsilon}^{c}}(f-\lambda-\epsilon) \geq 0$. We let $\mathcal{K}:=\cap_{\epsilon>0} \mathcal{K}_{\epsilon}$. We also say that a function $g$ or $f$ as above is inf-compact if it is near-monotone relative to all $\lambda \in \mathbb{R}$.

Note that the concept of near-monotonicity in the literature is often stricter - a function $f$ is sometimes called near-monotone if it is near-monotone relative to all $\lambda<\|f\|_{\infty}[\underline{3}]$. 
For an inf-compact running cost $c$, which is what we most often see in applications, near-monotonicity is of course equivalent to the statement that $\Lambda^{*}<\infty$. Therefore, for a inf-compact running cost, nearmonotonicity is also necessary for the optimal control problem to be well posed. There are clearly two tasks for this class of models. First, establish that the class of stabilizing Markov controls $\mathfrak{U}_{\text {stab }}$ is nonempty, and then solve the optimal control problem. This paper addresses the second task.

The main results of the paper can be divided into two groups. Those concerning the risk-sensitive control problem, and those concerning the multiplicative Poisson equation (MPE) for (uncontrolled) diffusions.

For the risk sensitive control problem, there are two sets of results. First, under the hypothesis that the running cost $c$ is near-monotone relative to $\Lambda^{*}$, and an assumption on the drift that limits but not precludes transience of the controlled process (see Assumption 1.1), we establish existence of a solution to the risksensitive HJB equation, and also existence of a stationary Markov control which is optimal over the class of all admissible controls (see Proposition 1.1). We wish to point out the optimality over nonstationary controls is very hard to obtain for the risk-sensitive problem without blanket geometric ergodicity hypotheses. For this reason, the optimal control problem is often restricted to stationary Markov controls (see the analogous study in the case of denumerable controlled Markov chains in [15]).

If the running cost is near-monotone relative to $\Lambda_{\mathrm{m}}^{*}$, then, without any additional assumptions on the drift, we show in Proposition 1.3 that there exists a pair $\left(V^{*}, \Lambda_{\mathrm{m}}^{*}\right) \in \mathcal{C}^{2}\left(\mathbb{R}^{d}\right) \times \mathbb{R}$ solving the HJB equation and any measurable selector of the HJB is a stable control, and is optimal in the class of stationary Markov controls. Under the same near-monotonicity hypothesis, together with the assumption that $c$ is inf-compact, the risk-sensitive problem for denumerable Markov decision processes is treated in [15], where a dynamic programming inequality is established.

Concerning uniqueness of the solution to the HJB we identify a rather generic sufficient condition which amounts to strict monotonicity on the right for $\Lambda_{\mathrm{m}}^{*}$ with respect to the running cost $c$, i.e., that $c \supsetneqq c^{\prime}$ implies $\Lambda_{\mathrm{m}}^{*}(c)<\Lambda_{\mathrm{m}}^{*}\left(c^{\prime}\right)$. Under this condition, we show in Theorem 1.2 that there exists a unique solution to the HJB equation (up to a multiplicative constant), and we have the usual verification result that states that a stationary Markov control is optimal only if it is an a.e. measurable selector from the minimizer of the HJB. In addition, this condition is necessary and sufficient for the solution of the HJB to be the minimal solution of the MPE over the class of optimal stationary Markov controls.

The second set of results, which comprises a significant portion of the paper, concerns the MPE. Here, the running cost takes the role of a potential $f$ which satisfies the near-monotonicity hypothesis in Definition 1.1 relative to the principal eigenvalue $\Lambda^{*}(f)$. We present a comprehensive study of the relationship between the solutions of the MPE and their stochastic representations, and the recurrence properties of the so called twisted process (see [36]) which is associated with eigenfunctions of the principal eigenvalue $\Lambda^{*}(f)$. In the quantum mechanics literature this eigenfunction is called the ground state, and the twisted process is described by a diffusion which we refer to as the ground state diffusion (see (1.17)). The key results are in Theorems 1.5 1.6. These should be compared with the results for countable Markov chains in [3]. An important contribution of this paper is the sharp characterization of the recurrence properties of the ground state diffusion in terms of the monotonicity of the principal eigenvalue as a function of the potential.

The notation used in the paper is summarized in Section 1.1. The assumptions on the model are in Section 1.2. followed by a summary of the main results in Section 1.3. followed by a description of the model in Section 2 contains various results on the multiplicative Poisson equation, which lead to the proof of Theorems 1.5 1.8. In Section 1.4 we summarize some basic results from the theory of second order elliptic partial differential equations (pde) used in this paper. The proofs of the results concerning the risk-sensitive control problem are in Section 3 .

\subsection{Notation}

The standard Euclidean norm in $\mathbb{R}^{d}$ is denoted by $|\cdot|$, and $\langle\cdot, \cdot\rangle$ denotes the inner product. The set of nonnegative real numbers is denoted by $\mathbb{R}_{+}, \mathbb{N}$ stands for the set of natural numbers, and $\mathbb{1}$ denotes the indicator function. Given two real numbers $a$ and $b$, the minimum (maximum) is denoted by $a \wedge b(a \vee b)$, respectively. The closure, boundary, and the complement of a set $A \subset \mathbb{R}^{d}$ are denoted by $\bar{A}, \partial A$, and $A^{c}$, 
respectively. We denote by $\tau(A)$ the first exit time of the process $\left\{X_{t}\right\}$ from the set $A \subset \mathbb{R}^{d}$, defined by

$$
\tau(A):=\inf \left\{t>0: X_{t} \notin A\right\} .
$$

The open ball of radius $r$ in $\mathbb{R}^{d}$, centered at the origin, is denoted by $B_{r}$, and we let $\tau_{r}:=\tau\left(B_{r}\right)$, and $\breve{\tau}_{r}:=\tau\left(B_{r}^{c}\right)$.

The term domain in $\mathbb{R}^{d}$ refers to a nonempty, connected open subset of the Euclidean space $\mathbb{R}^{d}$. For a domain $D \subset \mathbb{R}^{d}$, the space $\mathcal{C}^{k}(D)\left(\mathcal{C}^{\infty}(D)\right)$ refers to the class of all real-valued functions on $D$ whose partial derivatives up to order $k$ (of any order) exist and are continuous, and $\mathcal{C}_{b}(D)$ denotes the set of all bounded continuous real-valued functions on $D$. In addition $\mathcal{C}_{c}(D)$ denotes the class of functions in $\mathcal{C}(D)$ that have compact support, and $\mathcal{C}_{0}\left(\mathbb{R}^{d}\right)$ the class of continuous functions on $\mathbb{R}^{d}$ that vanish at infinity. By a slight abuse of notation, whenever the whole space $\mathbb{R}^{d}$ is concerned, we write $f \in \mathcal{C}^{k}\left(\mathbb{R}^{d}\right)$ whenever $f \in \mathcal{C}^{k}(D)$ for all bounded domains $D \subset \mathbb{R}^{d}$. The space $L^{p}(D), p \in[1, \infty)$, stands for the Banach space of (equivalence classes of) measurable functions $f$ satisfying $\int_{D}|f(x)|^{p} \mathrm{~d} x<\infty$, and $L^{\infty}(D)$ is the Banach space of functions that are essentially bounded in $D$. The standard Sobolev space of functions on $D$ whose generalized derivatives up to order $k$ are in $L^{p}(D)$, equipped with its natural norm, is denoted by $\mathcal{W}^{k, p}(D)$, $k \geq 0, p \geq 1$.

In general, if $\mathcal{X}$ is a space of real-valued functions on $Q, \mathcal{X}_{\text {loc }}$ consists of all functions $f$ such that $f \varphi \in \mathcal{X}$ for every $\varphi \in \mathcal{C}_{c}^{\infty}(Q)$, the space of smooth functions on $Q$ with compact support. In this manner we obtain for example the space $\mathcal{W}_{\text {loc }}^{2, p}(Q)$.

For a continuous function $g: \mathbb{R}^{d} \rightarrow[1, \infty)$ we let $L_{g}^{\infty}($ or $\mathcal{O}(g)$ ) denote the space of Borel measurable functions $f: \mathbb{R}^{d} \rightarrow \mathbb{R}$ satisfying

$$
\underset{x \in \mathbb{R}^{d}}{\operatorname{esssup}} \frac{|f(x)|}{g(x)}<\infty,
$$

and by $\mathfrak{o}(g)$ the subspace of functions $f \in L_{g}^{\infty}$ such that

$$
\limsup _{R \rightarrow \infty} \operatorname{esssup}_{x \in B_{R}^{c}} \frac{|f(x)|}{g(x)}=0 .
$$

We also let $\mathcal{C}_{g}\left(\mathbb{R}^{d}\right)$ denote the Banach space of continuous functions under the norm

$$
\|f\|_{g}:=\sup _{x \in \mathbb{R}^{d}} \frac{|f(x)|}{g(x)} .
$$

We adopt the notation $\partial_{i}:=\frac{\partial}{\partial x_{i}}$ and $\partial_{i j}:=\frac{\partial^{2}}{\partial x_{i} \partial x_{j}}$ for $i, j \in \mathbb{N}$. We often use the standard summation rule that repeated subscripts and superscripts are summed from 1 through $d$. For example,

$$
\frac{1}{2} a^{i j} \partial_{i j} \varphi+b^{i} \partial_{i} \varphi:=\frac{1}{2} \sum_{i, j=1}^{d} a^{i j} \frac{\partial^{2} \varphi}{\partial x_{i} \partial x_{j}}+\sum_{i=1}^{d} b^{i} \frac{\partial \varphi}{\partial x_{i}} .
$$

\subsection{The model}

The following assumptions on the diffusion (1.1) are in effect throughout the paper unless otherwise mentioned.

(A1) Local Lipschitz continuity: The functions

$$
b=\left[b^{1}, \ldots, b^{d}\right]^{\top}: \mathbb{R}^{d} \times \mathbb{U} \rightarrow \mathbb{R}^{d}, \quad \text { and } \quad \sigma=\left[\sigma^{i j}\right]: \mathbb{R}^{d} \rightarrow \mathbb{R}^{d \times d}
$$

are locally Lipschitz in $x$ with a Lipschitz constant $C_{R}>0$ depending on $R>0$. In other words, we have

$$
|b(x, u)-b(y, u)|+\|\sigma(x)-\sigma(y)\| \leq C_{R}|x-y| \quad \forall x, y \in B_{R}, \quad \text { and } u \in \mathbb{U} .
$$

We also assume that $b$ is continuous. 
(A2) Affine growth condition: $b$ and $\sigma$ satisfy a global growth condition of the form

$$
|b(x, u)|^{2}+\|\sigma(x)\|^{2} \leq C_{0}\left(1+|x|^{2}\right) \quad \forall(x, u) \in \mathbb{R}^{d} \times \mathbb{U}
$$

for some constant $C_{0}>0$, where $\|\sigma\|^{2}:=\operatorname{trace}\left(\sigma \sigma^{\top}\right)$.

(A3) Nondegeneracy: For each $R>0$, it holds that

$$
\sum_{i, j=1}^{d} a^{i j}(x) \xi_{i} \xi_{j} \geq C_{R}^{-1}|\xi|^{2} \quad \forall x \in B_{R},
$$

and for all $\xi=\left(\xi_{1}, \ldots, \xi_{d}\right)^{\top} \in \mathbb{R}^{d}$, where $a:=\sigma \sigma^{\top}$.

In integral form, (1.1) is written as

$$
X_{t}=X_{0}+\int_{0}^{t} b\left(X_{s}, U_{s}\right) \mathrm{d} s+\int_{0}^{t} \sigma\left(X_{s}\right) \mathrm{d} W_{s} .
$$

The third term on the right hand side of (1.3) is an Itô stochastic integral. We say that a process $X=$ $\left\{X_{t}(\omega)\right\}$ is a solution of (1.1), if it is $\mathfrak{F}_{t}$-adapted, continuous in $t$, defined for all $\omega \in \Omega$ and $t \in[0, \infty)$, and satisfies (1.3) for all $t \in[0, \infty)$ a.s. It is well known that under (A1)-(A3), for any admissible control there exists a unique solution of (1.1) 2, Theorem 2.2.4]. We define the family of operators $\mathcal{L}^{u}: \mathcal{C}^{2}\left(\mathbb{R}^{d}\right) \mapsto \mathcal{C}\left(\mathbb{R}^{d}\right)$, where $u \in \mathbb{U}$ plays the role of a parameter, by

$$
\mathcal{L}^{u} f(x)=\frac{1}{2} a^{i j}(x) \partial_{i j} f(x)+b^{i}(x, u) \partial_{i} f(x), \quad u \in \mathbb{U} .
$$

Let $\mathfrak{U}_{\mathrm{SM}}$ denote the set of stationary Markov controls. It is well known that under $v \in \mathfrak{U}_{\mathrm{SM}}$ (1.1) has a unique strong solution [30]. Moreover, under $v \in \mathfrak{U}_{\mathrm{SM}}$, the process $X$ is strong Markov, and we denote its transition kernel by $P_{v}^{t}(x, \cdot)$. It also follows from the work in [13] that under $v \in \mathfrak{U}_{\mathrm{SM}}$, the transition probabilities of $X$ have densities which are locally Hölder continuous. Thus $\mathcal{L}_{v}$ defined by

$$
\mathcal{L}_{v} f(x)=\frac{1}{2} a^{i j}(x) \partial_{i j} f(x)+b^{i}(x, v(x)) \partial_{i} f(x), \quad v \in \mathfrak{U}_{\mathrm{SM}},
$$

for $f \in \mathcal{C}^{2}\left(\mathbb{R}^{d}\right)$, is the generator of a strongly-continuous semigroup on $\mathcal{C}_{b}\left(\mathbb{R}^{d}\right)$, which is strong Feller. When $v \in \mathfrak{U}_{\mathrm{SM}}$ we use $v$ as subscript in $\mathcal{L}_{v}$ to distinguish it from $\mathcal{L}^{u}, u \in \mathbb{U}$, defined in the preceding paragraph. We let $\mathbb{P}_{x}^{v}$ denote the probability measure and $\mathbb{E}_{x}^{v}$ the expectation operator on the canonical space of the process under the control $v \in \mathfrak{U}_{\mathrm{SM}}$, conditioned on the process $X$ starting from $x \in \mathbb{R}^{d}$ at $t=0$. We denote by $\mathfrak{U}_{\mathrm{SSM}}$ the subset of $\mathfrak{U}_{\mathrm{SM}}$ that consists of stable controls, i.e., under which the controlled process is positive recurrent, and by $\mu_{v}$ the invariant probability measure of the process under the control $v \in \mathfrak{U}_{\mathrm{SSM}}$.

\subsection{Main results}

Consider the following assumption on the drift of (1.1).

Assumption 1.1. The coefficients $b$ and $\sigma$ of $\mathcal{L}^{u}$ in (1.4) are bounded, $\sigma$ is Lipschitz continuous, and for some constant $C$ we have

$$
\sum_{i, j=1}^{d} a^{i j}(x) \xi_{i} \xi_{j} \geq C^{-1}|\xi|^{2} \quad \forall x \in \mathbb{R}^{d} .
$$

In addition we assume that

$$
\max _{u \in \mathbb{U}} \frac{\langle b(x, u), x\rangle^{+}}{|x|} \underset{|x| \rightarrow \infty}{\longrightarrow} 0 .
$$


Recall the definitions in (1.2). We let $\mathcal{C}_{\circ}$ denote the class of nonnegative functions in $\mathcal{C}_{0}\left(\mathbb{R}^{d}\right)$ which are not identically equal to 0 . We also define

and

$$
\mathfrak{V}:=\left\{(V, \Lambda) \in \mathcal{C}^{2}\left(\mathbb{R}^{d}\right) \times \mathbb{R}: V(0)=1, V>0, \Lambda \leq \Lambda^{*}\right\}
$$

$$
\mathfrak{V}_{\circ}:=\left\{(V, \Lambda) \in \mathcal{C}^{2}\left(\mathbb{R}^{d}\right) \times \mathbb{R}: V(0)=1, \inf _{\mathbb{R}^{d}} V>0, \Lambda \leq \Lambda^{*}\right\}
$$

Proposition 1.1. Let $a$ and $b$ satisfy Assumption 1.1 and $c$ be near-monotone relative to $\Lambda^{*}$ and bounded. Then the HJB equation

$$
\min _{u \in \mathbb{U}}\left[\mathcal{L}^{u} V^{*}(x)+c(x, u) V^{*}(x)\right]=\Lambda^{*} V^{*}(x) \quad \forall x \in \mathbb{R}^{d}
$$

has a solution $V^{*} \in \mathcal{C}^{2}\left(\mathbb{R}^{d}\right)$, satisfying $V^{*}(0)=1$ and $\inf _{\mathbb{R}^{d}} V^{*}>0$, and the following hold:

(i) $\Lambda_{x}^{*}=\Lambda^{*}$ for all $x \in \mathbb{R}^{d}$.

(ii) Any $v \in \mathfrak{U}_{\mathrm{SM}}$ that satisfies

$$
\mathcal{L}_{v} V^{*}(x)+c(x, v(x)) V^{*}(x)=\min _{u \in \mathbb{U}}\left[\mathcal{L}^{u} V^{*}(x)+c(x, u) V^{*}(x)\right] \quad \text { a.e. } x \in \mathbb{R}^{d}
$$

is stable, and is optimal, i.e., $\Lambda_{x}^{v}=\Lambda^{*}$ for all $x \in \mathbb{R}^{d}$. In particular, $\Lambda_{\mathrm{m}}^{*}=\Lambda^{*}$.

(iii) It holds that

$$
V^{*}(x)=\mathbb{E}_{x}^{v}\left[\mathrm{e}^{\int_{0}^{T}\left[c\left(X_{t}, v\left(X_{t}\right)\right)-\Lambda^{*}\right] \mathrm{d} t} V^{*}\left(X_{T}\right)\right] \quad \forall(T, x) \in \mathbb{R}_{+} \times \mathbb{R}^{d},
$$

for any $v \in \mathfrak{U}_{\mathrm{SM}}$ that satisfies (1.7).

Proof. Existence of a solution and parts (i)-(ii) of the proposition follow by Theorem 3.4 in Section 3 Part (iii) follows by Theorem 1.5 and Lemma 3.3. together with the fact that a diffusion with Lipschitz continuous diffusion matrix and a drift having at most linear growth is regular.

Remark 1.1. The hypothesis in (1.5) of Assumption 1.1 may be replaced by the following. There exists a

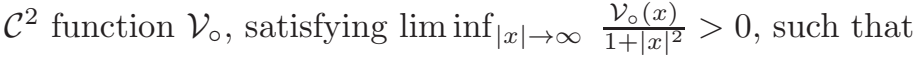

$$
\frac{\left[\mathcal{L}^{u} \mathcal{V}_{\circ}(x)\right]^{+}}{\sqrt{\mathcal{V}_{\circ}(x)}} \underset{|x| \rightarrow \infty}{\longrightarrow} 0 \quad \forall u \in \mathbb{U}
$$

It is clear from the proof that the result of Lemma 3.2 in Section 3 holds under this assumption. It is also evident that (1.5) may be replaced by the more general hypothesis that $\mathbb{E}_{x}^{U}\left[\left|X_{t}\right|\right] \in \mathfrak{o}(t)$ under any $U \in \mathfrak{U}$, which is the conclusion of Lemma 3.2 on which the proof of Theorem 3.4 is based. Note that when the coefficients $b$ and $\sigma$ are bounded, it is always the case that $\mathbb{E}_{x}^{U}\left[\left|X_{t}\right|\right] \in \mathcal{O}(t)$.

Remark 1.2. As shown in [7, Proposition 2.6] if $a$ and $b$ are bounded then in general $\Lambda^{*}=\infty$ when $c$ is not bounded. Therefore, the assumption that $c$ is bounded in Proposition 1.1 cannot be relaxed.

Let

$$
\mathfrak{U}_{\mathrm{SM}}^{*}:=\left\{v \in \mathfrak{U}_{\mathrm{SM}}: \Lambda_{x}^{v}(c)=\Lambda_{\mathrm{m}}^{*}, \forall x \in \mathbb{R}^{d}\right\} .
$$

Concerning uniqueness of the HJB equation we have the following. 
Theorem 1.2. Suppose that in addition to the assumptions of Proposition 1.1 it holds that

$$
\Lambda_{\mathrm{m}}^{*}(c+h)>\Lambda_{\mathrm{m}}^{*}(c) \quad \forall h \in \mathcal{C}_{\circ} .
$$

Then there exists a unique pair $(V, \Lambda) \in \mathfrak{V}$ which solves

$$
\min _{u \in \mathbb{U}}\left[\mathcal{L}^{u} V(x)+c(x, u) V(x)\right]=\Lambda V(x) \quad \forall x \in \mathbb{R}^{d} .
$$

and $v \in \mathfrak{U}_{\mathrm{SM}}^{*}$ if and only if it satisfies (1.7). In addition, the function $V^{*}$ in Proposition 1.1 has the stochastic representation

$$
V^{*}(x)=\mathbb{E}_{x}^{v}\left[\mathrm{e}^{\int_{0}^{\breve{\tau}_{r}}\left[c\left(X_{t}, v\left(X_{t}\right)\right)-\Lambda^{*}\right] \mathrm{d} t} V^{*}\left(X_{\breve{\tau}_{r}}\right)\right] \quad \forall x \in \bar{B}_{r}^{c},
$$

for all $r>0$, and $v \in \mathfrak{U}_{\mathrm{SM}}^{*}$. Conversely, if $v \in \mathfrak{U}_{\mathrm{SM}}^{*}$ satisfies (1.10) for some $r>0$, then $\Lambda^{v}(c+h)>\Lambda^{v}(c)$ for all $h \in \mathrm{C}_{\circ}$.

Proof. The proof is in Section 3

Without imposing any restrictions on the coefficients, we have the following result.

Proposition 1.3. Suppose that $c$ is near-monotone relative to $\Lambda_{\mathrm{m}}^{*}$. Then the HJB equation

$$
\min _{u \in \mathbb{U}}\left[\mathcal{L}^{u} V^{*}(x)+c(x, u) V^{*}(x)\right]=\Lambda_{\mathrm{m}}^{*} V^{*}(x) \quad \forall x \in \mathbb{R}^{d}
$$

has a solution $V^{*} \in \mathcal{C}^{2}\left(\mathbb{R}^{d}\right)$, satisfying $V^{*}(0)=1$ and $\inf _{\mathbb{R}^{d}} V^{*}>0$. Moreover, any $v \in \mathfrak{U}_{\mathrm{SM}}$ that satisfies (1.7) is stable, and is optimal in the class $\mathfrak{U}_{\mathrm{SM}}$, i.e., $\Lambda_{x}^{v}=\Lambda_{\mathrm{m}}^{*}$ for all $x \in \mathbb{R}^{d}$.

Under the additional assumption in (1.8), there exists a unique pair $(V, \Lambda) \in \mathfrak{V}_{\circ}$ which solves (1.9), and $v \in \mathfrak{U}_{\mathrm{SM}}^{*}$ if and only if it satisfies (1.7). The function $V^{*}$ has the stochastic representation

$$
V^{*}(x)=\mathbb{E}_{x}^{v}\left[\mathrm{e}^{\int_{0}^{\check{\tau}_{r}}\left[c\left(X_{t}, v\left(X_{t}\right)\right)-\Lambda_{\mathrm{m}}^{*}\right] \mathrm{d} t} V^{*}\left(X_{\breve{\tau}_{r}}\right)\right] \quad \forall x \in \bar{B}_{r}^{c},
$$

for all $r>0$, and $v \in \mathfrak{U}_{\mathrm{SM}}^{*}$. Conversely, if $v \in \mathfrak{U}_{\mathrm{SM}}^{*}$ satisfies (1.11) for some $r>0$, then $\Lambda^{v}(c+h)>\Lambda^{v}(c)$ for all $h \in \mathcal{C}_{0}$.

The proof of Proposition 1.3 is in Section 3.

Remark 1.3. The main reason $\Lambda_{\mathrm{m}}^{*}$ appears in Proposition 1.3 instead of $\Lambda^{*}$ has to do with the way the solution $V^{*}$ is constructed. It should be kept in mind that $\Lambda^{*} \leq \Lambda_{\mathrm{m}}^{*}$, in general, and therefore we cannot follow the path of Proposition 1.1 to prove Proposition 1.3 Instead, a fixed $\epsilon$-optimal stationary Markov control is imposed at ' $\infty$ ' to guarantee that the solution is bounded away from zero, and then a limit is taken as $\epsilon \searrow 0$. This has the effect of restricting optimality over the class $\mathfrak{U}_{\mathrm{SM}}$. For more details on this see Remark 3.2 in Section 3

The proofs of these results depend heavily on properties of the multiplicative Poisson equation (MPE), which are summarized next.

\subsubsection{Results concerning the multiplicative Poisson equation}

We consider an uncontrolled diffusion

$$
\mathrm{d} X_{t}=b\left(X_{t}\right) \mathrm{d} t+\sigma\left(X_{t}\right) \mathrm{d} W_{t},
$$

where $\sigma$ and $b$ satisfy (A2)-(A3), $\sigma$ is locally Lipschitz (as in (A1)), and $b$ is measurable. We let $\mathbb{E}_{x}$ denote the expectation operator induced by the strong Markov process with $X_{0}=x$, governed by (1.12), and

$$
\mathcal{L}:=\frac{1}{2} a^{i j}(x) \partial_{i j}+b^{i}(x) \partial_{i},
$$


with $a:=\sigma \sigma^{\top}$. Let $f: \mathbb{R}^{d} \rightarrow \mathbb{R}_{+}$be measurable and locally bounded, and define

$$
\begin{aligned}
\Lambda_{x}(f) & :=\limsup _{T \rightarrow \infty} \frac{1}{T} \log \mathbb{E}_{x}\left[\mathrm{e}^{\int_{0}^{T} f\left(X_{t}\right) \mathrm{d} t}\right] \quad \forall x \in \mathbb{R}^{d}, \\
\Lambda(f) & :=\inf _{x \in \mathbb{R}^{d}} \Lambda_{x}(f) .
\end{aligned}
$$

We assume $\Lambda(f)<\infty$. We say that $(\Psi, \Lambda) \in \mathcal{W}_{\text {loc }}^{2, p}\left(\mathbb{R}^{d}\right) \times \mathbb{R}, p>d, \Psi>0$, is a solution of the multiplicative Poisson equation (MPE) if it satisfies

$$
\mathcal{L} \Psi(x)+f(x) \Psi(x)=\Lambda \Psi(x) \quad \text { a.e. } x \in \mathbb{R}^{d} .
$$

We refer to $\Lambda$ as an eigenvalue of the MPE.

Consider the following hypothesis.

(H1) The diffusion in (1.12) is recurrent, and $f: \mathbb{R}^{d} \rightarrow \mathbb{R}_{+}$is a locally bounded measurable map which is near-monotone relative to $\Lambda(f)$.

Implicit in the statement in (H1) is of course the requirement $\Lambda(f)<\infty$. We have assumed $f \geq 0$, for simplicity, but all the results are valid with $f$ bounded below in $\mathbb{R}^{d}$.

We compare the definition in (1.14) with the following definitions for the principal eigenvalue, commonly used in the pde literature [7]:

$$
\begin{aligned}
& \hat{\Lambda}(f):=\inf \left\{\lambda \in \mathbb{R}: \exists \varphi \in \mathcal{W}_{\text {loc }}^{2, d}\left(\mathbb{R}^{d}\right), \varphi>0, \mathcal{L} \varphi+(f-\lambda) \varphi \leq 0 \text { a.e. in } \mathbb{R}^{d}\right\}, \\
& \hat{\Lambda}(f):=\inf \left\{\lambda \in \mathbb{R}: \exists \varphi \in \mathcal{W}_{\text {loc }}^{2, d}\left(\mathbb{R}^{d}\right), \inf _{\mathbb{R}^{d}} \varphi>0, \mathcal{L} \varphi+(f-\lambda) \varphi \leq 0 \text { a.e. in } \mathbb{R}^{d}\right\} .
\end{aligned}
$$

We have the following theorem.

Theorem 1.4. Under (H1), we have $\Lambda(f)=\hat{\Lambda}(f)=\hat{\Lambda}(f)$.

Proof. The proof is in Section 2.1

Definition 1.2. Let $\Psi \in \mathcal{W}_{\text {loc }}^{2, p}\left(\mathbb{R}^{d}\right), p>d$, be a positive solution of the MPE

$$
\mathcal{L} \Psi(x)+f(x) \Psi(x)=\Lambda(f) \Psi(x) \quad \text { a.e. } x \in \mathbb{R}^{d},
$$

and let $\psi:=\log \Psi$. We introduce the stochastic differential equation (sde)

$$
\mathrm{d} X_{t}^{*}=\left(b\left(X_{t}^{*}\right)+a\left(X_{t}^{*}\right) \nabla \psi\left(X_{t}^{*}\right)\right) \mathrm{d} t+\sigma\left(X_{t}^{*}\right) \mathrm{d} W_{t}^{*},
$$

where $W^{*}$ is, as usual, a standard Wiener process. We denote by $\mathcal{L}^{*}$ the extended generator of (1.17), given by

$$
\mathcal{L}^{*} g:=\frac{1}{2} a^{i j} \partial_{i j} g+\langle b, \nabla g\rangle+\langle a \nabla \psi, \nabla g\rangle
$$

for $g \in \mathcal{C}^{2}\left(\mathbb{R}^{d}\right)$.

The sde in (1.17) is well known. Recall the Feynman-Kac semigroup corresponding to $\mathcal{L}+f$, given by

$$
P_{t}^{f} h(x):=\mathbb{E}_{x}\left[\mathrm{e}^{\int_{0}^{t} f\left(X_{s}\right) \mathrm{d} s} h\left(X_{t}\right)\right] \quad \text { for } h \geq 0, \quad h \text { measurable. }
$$

The function $f$ is referred to as the potential in the study of the Feynman-Kac semigroup for symmetric Markov processes, and the eigenfunction $\Psi$ is called a ground state. The ground state semigroup is given by

$$
\mathcal{T}_{t}^{\Psi} h(x):=\mathrm{e}^{-\Lambda(f) t} \frac{1}{\Psi(x)} P_{t}^{f}(\Psi h)(x),
$$


and it turns out that $\mathcal{L}^{*}$ is its generator [45, 51]. In [3, 36 $\mathcal{T}_{t}^{\Psi}$ called the twisted kernel. In addition, elliptic equations with $\mathcal{L}^{*}$ have been studied extensively in [35], albeit under smoothness assumptions on the coefficients.

Since the drift of (1.17) does not necessarily satisfy (A2), existence and uniqueness of a solution for this equation is not guaranteed. Diffusions with locally bounded drift have been studied in 30], and under the assumption that the diffusion matrix $\sigma$ is locally Lipschitz and nonsingular, existence of a unique strong solution up to explosion time has been established. Recently, similar results have been obtained for locally integrable drifts [39]. Moreover, if the diffusion in (1.17) is positive recurrent, it is well known that it has a unique invariant probability measure, with a positive density [13].

We say that the diffusion in (1.17) is regular, if it has a unique strong solution which exists for all $t>0$.

Theorem 1.5. Assume (H1). Then the diffusion in 1.17) is regular if and only if $P_{t}^{f} \Psi(x)=\mathrm{e}^{\Lambda(f) t} \Psi(x)$ for all $(t, x) \in \mathbb{R}_{+} \times \mathbb{R}^{d}$. In addition, the following are equivalent:

(i) The process $X^{*}$ in (1.17) is recurrent.

(ii) For some $r>0$, we have

$$
\Psi(x)=\mathbb{E}_{x}\left[\mathrm{e}^{\int_{0}^{\breve{\tau}_{r}}\left[f\left(X_{s}\right)-\Lambda(f)\right] \mathrm{d} s} \Psi\left(X_{\breve{\tau}_{r}}\right)\right] \quad \forall x \in B_{r}^{c} .
$$

(iii) For some $x \in \mathbb{R}^{d}$ it holds that

$$
\int_{0}^{\infty} \mathbb{E}_{x}\left[\mathrm{e}^{\int_{0}^{t}\left[f\left(X_{s}\right)-\Lambda(f)\right] \mathrm{d} s}\right] \mathrm{d} t=\infty
$$

(iv) It holds that $\Lambda(f)<\Lambda(f+h)$ for all $h \in \mathcal{C}_{\circ}$.

(v) If $A \subset \mathbb{R}^{d}$ is a measurable set of positive Lebesgue measure, then $\Lambda(f)<\Lambda\left(f+\epsilon \mathbb{1}_{A}\right)$ for all $\epsilon>0$.

Moreover, in (ii)-(iii) "some" may be replaced with "all".

It is clear by Theorem 1.5(ii) that if the process $X^{*}$ in (1.17), corresponding to some positive solution $\Psi$ of $(1.16)$, is recurrent, then there exists a unique positive solution $\Psi \in \mathcal{W}_{\text {loc }}^{2, p}\left(\mathbb{R}^{d}\right), p>d$, to the MPE in (1.16), satisfying $\Psi(0)=1$. In particular the ground state diffusion in (1.17) is uniquely determined by $f$.

Proof. These results are contained in individual lemmas in Section 2.2. The first assertion follows by Lemmas 2.4 and 2.5. That (i) $\Leftrightarrow$ (ii) and (i) $\Leftrightarrow$ (v) follow by Lemmas 2.6 and 2.11, respectively. Theorem 2.8 asserts that (iii) $\Rightarrow$ (ii), while (i) $\Rightarrow$ (iii) follows by Lemma 2.9. It is easy to see that (v) $\Rightarrow$ (iv), and by Lemma 2.11, (iv) $\Rightarrow$ (i).

Theorem 1.6. Under (H1), the following are equivalent.

(i) The process $X^{*}$ in (1.17) is geometrically ergodic.

(ii) For some $r>0$, there exists $\delta_{r}>0$, such that

$$
\mathbb{E}_{x}\left[\mathrm{e}^{\int_{0}^{\breve{\tau}_{r}}\left[f\left(X_{s}\right)-\Lambda(f)+\delta_{r}\right] \mathrm{d} s}\right]<\infty \quad \forall x \in \bar{B}_{r}^{c} .
$$

(iii) It holds that $\Lambda(f)>\Lambda(f-h)$ for some $h \in \mathcal{C}_{\circ}$.

Moreover, in (ii)-(iii) "some" may be replaced with "all".

Proof. This follows by Theorem 2.12 and Corollary 2.14 in Section 2.3 
Remark 1.4. Strict monotonicity of the principal eigenvalue, i.e., $f^{\prime} \supsetneqq f$ implies $\Lambda\left(f^{\prime}\right)<\Lambda(f)$, holds for bounded domains (see also Lemma 2.2(b) in Section 2). However, this property does not hold in unbounded domains in general [7, Remark 2.4]. Under the near-monotone hypothesis in (H1), Theorems 1.5 1.6 provide the following characterization: Let $X^{*}$ be the process in (1.17) corresponding to a solution of (1.16) for $f$. Then $f \supsetneqq f^{\prime}$ implies $\Lambda\left(f^{\prime}\right)<\Lambda(f)$ if and only if $X^{*}$ is geometrically ergodic, and $X^{*}$ is recurrent if and only if $\Lambda\left(f^{\prime}\right)>\Lambda(f)$ for all $f^{\prime}$ satisfying $f^{\prime} \supsetneqq f$.

Generally speaking, not much is known for the principal eigensolutions in $\mathbb{R}^{d}$ of the class of elliptic operators $\mathcal{L}$ considered in this paper. Compare with Sections 8-9 in [7]. Therefore we feel this characterization will be of interest to a wider audience.

Remark 1.5. It is evident by Theorem 1.5 (iv) and (iii) $\Rightarrow$ (i) in Theorem 1.6 that if the ground state diffusion corresponding to $f$ is recurrent, then the ground state diffusion corresponding to $f+h$ is geometrically ergodic for any $h \in \mathcal{C}_{\circ}$.

It is also interesting to note that, under (H1), there always exists $h \in \mathcal{C}_{\circ}$ such that the ground state diffusion in (1.17) corresponding to $f+h$ is geometrically ergodic. In fact such an $h$ may be selected in $\mathcal{C}_{c}\left(\mathbb{R}^{d}\right)$ with a given support. This assertion follows by the proof of Lemma 2.11.

Consider the following hypothesis.

(H2) There exists a smooth function $\psi_{0}$ such that

$$
\frac{1}{2} a^{i j} \partial_{i j} \psi_{0}+\left\langle b, \nabla \psi_{0}\right\rangle+\frac{1}{2}\left\langle\nabla \psi_{0}, a \nabla \psi_{0}\right\rangle+f \rightarrow-\infty \quad \text { as } \quad|x| \rightarrow \infty .
$$

In Theorem 1.7 stated below, we do not assume that the running cost is inf-compact, or even nearmonotone in the sense of [3, p. 126]. Nor do we assume that $\Lambda(\alpha f)<\infty$ for some $\alpha>1$, as is common in the literature. This should be compared with 3, Theorem 1.2], and [15, Theorem 2.2] for irreducible Markov chains, as well as the more general results in 36, 37, 51.

Theorem 1.7. Under (H1)-(H2), the sde in (1.17) has a unique strong solution $X^{*}$ which exists for all $t>0$ and is a strong Markov process. Moreover, it is positive recurrent, and its unique invariant probability measure $\mu^{*}$ has positive density. In addition, for any $g \in \mathcal{C}_{b}\left(\mathbb{R}^{d}\right)$, the following hold:

$$
\begin{aligned}
& \mathcal{T}_{t}^{\Psi} g(x)=\mathbb{E}_{x}^{*}\left[g\left(X_{t}^{*}\right)\right] \quad \forall(t, x) \in \mathbb{R}_{+} \times \mathbb{R}^{d}, \\
& \mathcal{T}_{t}^{\Psi} g(x) \underset{t \rightarrow \infty}{\longrightarrow} \int_{\mathbb{R}^{d}} g(y) \mu^{*}(\mathrm{~d} y) \quad \forall x \in \mathbb{R}^{d},
\end{aligned}
$$

where $\mathbb{E}_{x}^{*}$ denotes the expectation operator associated with the solution $X^{*}$ of (1.17). In particular, the limit

$$
\Psi_{*}(x):=\lim _{t \rightarrow \infty} \mathbb{E}_{x}\left[\mathrm{e}^{\int_{0}^{t}\left[f\left(X_{s}\right)-\Lambda(f)\right] \mathrm{d} s}\right]
$$

is the unique positive solution of the MPE in (1.16) which satisfies $\mu^{*}\left(\Psi_{*}^{-1}\right)=1$.

Proof. These results are contained in Theorem 2.13 and Lemma 2.15 in Section 2.3.

Remark 1.6. The diffusion in (1.17) is studied extensively in 35]. A diffusion of the form

$$
\mathrm{d} X_{t}^{*}=\left(b\left(X_{t}^{*}\right)+\hat{a}\left(X_{t}^{*}\right) \nabla \breve{\psi}\left(X_{t}^{*}\right)\right) \mathrm{d} t+\sigma\left(X_{t}^{*}\right) \mathrm{d} W_{t}^{*}
$$

is treated, where $\breve{\psi}$ is a solution of (compare with (1.26) $)$

$$
\frac{1}{2} a^{i j} \partial_{i j} \breve{\psi}+\langle b, \nabla \breve{\psi}\rangle+\frac{1}{2}\langle\nabla \breve{\psi}, \hat{a} \nabla \breve{\psi}\rangle+f=\lambda
$$


The assumptions imposed are that $a, \hat{a}, b$ and $f$ are smooth and for some constant $C>0$ it holds that

$$
C^{-1}|\xi|^{2} \leq \sum_{i, j=1}^{d} \max \left\{a^{i j}(x), \hat{a}^{i j}(x)\right\} \xi_{i} \xi_{j} \leq C^{-1}|\xi|^{2} \quad \forall \xi, x \in \mathbb{R}^{d} .
$$

In addition they assume (1.21). Under these assumptions, they show that the set of $\lambda$ for which (1.23) has a solution is of the form $\left[\lambda_{*}, \infty\right)$, and that when $\lambda=\lambda_{*}$ the solution of (1.23) is unique and (1.22) is positive recurrent. In addition, for $\lambda>\lambda_{*},(1.22)$ is transient.

In comparing their results with ours, it is evident that (1.23) is more general than the Poisson equation considered here and there are no assumptions concerning near-monotonicity for $f$. On the other hand, we do not require any smoothness of the coefficients, nor do we require (1.24).

Next, we consider the following hypothesis.

(H3) For some $\varepsilon>0, \Lambda((1+\varepsilon) f)<\infty$, and $f$ is near monotone relative to $\frac{1}{\varepsilon}[\Lambda((1+\varepsilon) f)-\Lambda(f)]$.

If $f$ is inf-compact, then (H3) is equivalent to the statement that $\Lambda((1+\varepsilon) f)<\infty$. On the other hand, since $\frac{1}{\varepsilon}[\Lambda((1+\varepsilon) f)-\Lambda(f)] \geq \Lambda(f)$ by the convexity of $f \mapsto \Lambda(f)$ (see Lemma $2.2(\mathrm{~b})$ ), it follows that $(\mathrm{H} 3) \Rightarrow(\mathrm{H} 1)$. It thus follows from Definition 1.1, that under $(\mathrm{H} 3)(1+\varepsilon) f$ is near-monotone relative to $\Lambda((1+\varepsilon) f)$. We have the following theorem, whose proof is at the end of Section 2

Theorem 1.8. Under (H1) and (H3), the diffusion in (1.17) is geometrically ergodic, and there exist positive constants $\gamma, \kappa^{*}$ and $\beta^{*}$, depending on $\varepsilon$, such that if $g: \mathbb{R}^{d} \rightarrow \mathbb{R}$ is any locally bounded measurable function satisfying $\|g\|_{\Psi^{\gamma}}<\infty$, it holds that

$$
\left|\mathbb{E}_{x}^{*}\left[g\left(X_{t}\right)\right]-\mu^{*}(g)\right| \leq \kappa^{*} \mathrm{e}^{-\beta^{*} t}\|g\|_{\Psi^{\gamma}}\left(1+\Psi^{\gamma}(x)\right) \quad \forall t>0 .
$$

Moreover, if $f$ is inf-compact then $\gamma$ can be chosen arbitrarily close to $\varepsilon$.

Proof. The proof of this theorem is in Section 2.3.

Remark 1.7. Hypothesis (H3) is often used in the literature [3, 51]. As seen from Theorem [1.8, under (H3) the ground state diffusion (1.17) is geometrically ergodic with a 'storage function' $\Psi^{\gamma}$ for some $\gamma>0$. Nevertheless, even under (H2) the ground state diffusion is geometrically ergodic as can be seen by (2.45). The situation seems to be different for denumerable Markov chains where unless (H3) holds the twisted kernel cannot be geometrically ergodic [3, Theorem 5.1 (ii)].

In closing this section, let us mention that it is a direct consequence of Jensen's inequality that under (H1), the risk sensitive value $\Lambda(f)$ is not less that the ergodic value $\mu(f)$, where $\mu$ is the invariant probability measure of (1.12). The difference $\Lambda(f)-\mu(f)$ can be quantified as the following lemma shows. To accomplish this we use the equation arising from (1.15) under the transformation $\psi=\log \Psi$, which takes the form

$$
\frac{1}{2} a^{i j} \partial_{i j} \psi+\langle b, \nabla \psi\rangle+\frac{1}{2}\langle\nabla \psi, a \nabla \psi\rangle=\Lambda(f)-f .
$$

Lemma 1.9. Let $\mathcal{G}:=\langle\nabla \psi, a \nabla \psi\rangle$, with $\psi=\log \Psi$. Under (H1), it holds that

$$
\frac{1}{2} \mu(\mathcal{G})+\mu(f)=\Lambda(f),
$$

where $\mu$ is the invariant probability measure of (1.12).

PROOF. By Itô's formula, we obtain from (1.26)

$$
\mathbb{E}_{x}\left[\psi\left(X_{t \wedge \tau_{n}}\right)\right]-\psi(x)+\frac{1}{2} \mathbb{E}_{x}\left[\int_{0}^{t \wedge \tau_{n}} \mathcal{G}\left(X_{s}\right) \mathrm{d} s\right]+\mathbb{E}_{x}\left[\int_{0}^{t \wedge \tau_{n}} f\left(X_{s}\right) \mathrm{d} s\right]=\Lambda(f) \mathbb{E}_{x}\left[t \wedge \tau_{n}\right] .
$$


Since $f$ is near-monotone relative to $\Lambda(f)$, (2.3) takes the form $\mathcal{L} \Psi \leq k_{0}-k_{1} \Psi$ for some positive constants $k_{0}$ and $k_{1}$. It then follows by [2, Lemma 3.7.2], that any $h \in \mathfrak{o}(\Psi)$ satisfies

$$
\mathbb{E}_{x}\left[h\left(X_{t \wedge \tau_{n}}\right)\right] \underset{n \rightarrow \infty}{\longrightarrow} \mathbb{E}_{x}\left[h\left(X_{t}\right)\right], \quad \text { and } t^{-1} \mathbb{E}_{x}\left[h\left(X_{t}\right)\right] \underset{n \rightarrow \infty}{\longrightarrow} 0 .
$$

Since $f$ is bounded below, we may assume without loss of generality that it is nonnegative. We first take limits as $n \rightarrow \infty$ in (1.28), using (1.29) with $\psi \in \mathfrak{o}(\Psi)$ and monotone convergence for the integrals, then divide by $t$ and take limits as $t \rightarrow \infty$, using again (1.28) and Birkhoff's ergodic theorem to obtain (1.27).

\subsection{Some basic results from the theory of second order elliptic pdes}

In this paper we use some basic properties of elliptic pdes which we describe next. The first is Harnack's inequality that plays a central role in the study of elliptic equations, and can be stated as follows 29, Theorem 9.1]. Suppose that $\phi \in \mathcal{W}_{\text {loc }}^{2, p}\left(B_{R+1}\right), p>d, R>0$, is a positive function that solves $\mathcal{L} \phi+h \phi=0$ on $B_{R+1}$, with $h \in L^{\infty}\left(B_{R+1}\right)$. Then there exists a constant $C_{H}$ depending only on $R, d$, the constants $C_{R+1}$ and $C_{0}$ in (A1)-(A3), and $\|h\|_{\infty}$ such that

$$
\phi(x) \leq C_{H} \phi(y) \quad \forall x, y \in B_{R}
$$

Relative weak compactness of a family of functions in $\mathcal{W}_{\text {loc }}^{2, p}\left(B_{R+1}\right)$ can be obtained as a result of the following well-known a priori estimate [19, Lemma 5.3]. If $\varphi \in \mathcal{W}_{\mathrm{loc}}^{2, p}\left(B_{R+1}\right) \cap L^{p}\left(B_{R+1}\right)$, with $p \in(1, \infty)$, then

$$
\|\varphi\|_{\mathcal{W}^{2, p}\left(B_{R}\right)} \leq C\left(\|\varphi\|_{L^{p}\left(B_{R+1}\right)}+\|\mathcal{L} \varphi\|_{L^{p}\left(B_{R+1}\right)}\right)
$$

with the constant $C$ depending only on $d, R, C_{R+1}$, and $C_{0}$. This estimate along with the compactness of the embedding $\mathcal{W}^{2, d}\left(B_{R}\right) \hookrightarrow \mathcal{C}^{1, r}\left(\bar{B}_{R}\right)$, for $p>d$ and $r<1-\frac{d}{p}$ (see [19, Proposition 1.6]) imply the equicontinuity of any family of functions $\varphi_{n}$ which satisfies $\sup _{n}\left(\left\|\varphi_{n}\right\|_{L^{p}\left(B_{R+1}\right)}+\left\|\mathcal{L} \varphi_{n}\right\|_{L^{p}\left(B_{R+1}\right)}\right)<\infty, p>d$. We also frequently use the weak and strong maximum principles in the following form [29, Theorems 9.5 and 9.6, p. 225]. The weak maximum principle states that if $\varphi, \psi \in \mathcal{W}_{\text {loc }}^{2, d}(D) \cap \mathcal{C}(\bar{D})$ satisfy $(\mathcal{L}+h) \varphi=(\mathcal{L}+h) \psi$ in a bounded domain $D \subset \mathbb{R}^{d}$, with $h \in L^{d}(D), h \leq 0$ and $\varphi=\psi$ on $\partial D$, then $\varphi=\psi$ in $D$. On the other hand, the strong maximum principle states that if $\varphi \in \mathcal{W}_{\text {loc }}^{2, d}(D)$ satisfies $(\mathcal{L}+h) \varphi \leq 0$ in a bounded domain $D$, with $h=0(h \leq 0)$, then $\varphi$ cannot attain a minimum (nonpositive minimum) in $D$ unless it is a constant. We often use the following variation of the strong maximum principle. If $\varphi \geq 0$ and $(\mathcal{L}+h) \varphi \leq 0$ in a bounded domain $D \subset \mathbb{R}^{d}$, then $\varphi$ is either positive on $D$ or identically equal to 0 . This follows from the general statement by writing $(\mathcal{L}+h) \varphi \leq 0$ as

$$
\left(\mathcal{L}-h^{-}\right) \varphi \leq-h^{+} \varphi \leq 0 .
$$

In this paper, we use the theory of elliptic pdes to obtain limits of sequences of solutions to (1.15) as follows: Suppose $\varphi_{n} \in \mathcal{W}_{\text {loc }}^{2, p}\left(\mathbb{R}^{d}\right), p>d, n \in \mathbb{N}$, is a sequence of nonnegative functions satisfying

$$
\mathcal{L} \varphi_{n}+\left(f_{n}-\lambda_{n}\right) \varphi_{n}=-\alpha_{n} \quad \text { on } \mathbb{R}^{d}, \quad \forall n \in \mathbb{N},
$$

where $\left\{\lambda_{n}\right\}$ and $\left\{\alpha_{n}\right\}$ are bounded sequences of nonnegative real numbers, and $f_{n}$ is locally bounded and converges to some $f \in L_{\text {loc }}^{\infty}\left(\mathbb{R}^{d}\right)$ uniformly on compact sets. Suppose also that $\left\{\varphi_{n}(0)\right\}$ is a bounded sequence of positive numbers. Then by the extension of the Harnack inequality for a class of superharmonic functions in [1] (see also [2, Theorem A.2.13]) it follows that $\sup _{n \in \mathbb{N}}\left\|\varphi_{n}\right\|_{\infty, B_{R}}<\infty$ for every $R>0$. Thus, by the a priori estimate in (1.31) and the compactness of the embedding $\mathcal{W}^{2, d}\left(B_{R}\right) \hookrightarrow \mathcal{C}^{1, r}\left(\bar{B}_{R}\right)$, the sequence $\left\{\varphi_{n}\right\}$ along with its first derivatives are Hölder equicontinuous when restricted to any ball $B_{R}$. Thus, given any diverging sequence $\left\{k_{n}\right\} \subset \mathbb{N}$ we can extract a subsequence also denoted as $\left\{k_{n}\right\}$ along which $\varphi_{k_{n}}$ converges to some $\varphi \in \mathcal{W}_{\text {loc }}^{2, p}\left(\mathbb{R}^{d}\right), p>d$, and $\lambda_{k_{n}}$ and $\alpha_{k_{n}}$ converge to some constants $\lambda$ and $\alpha$ respectively, and satisfy

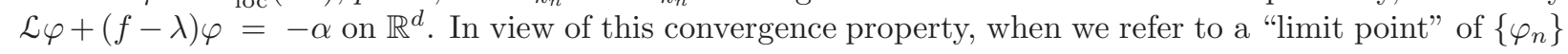
we mean a limit obtained as in the above procedure.

Completely analogous is the situation with solutions of (1.6). Moreover, since under the current assumptions, the map $x \mapsto \min _{u}[\langle b(x, u), \nabla \psi(x)\rangle+c(x, u) \psi(x)]$, is locally Hölder continuous, for any $\psi \in \mathcal{C}_{\text {loc }}^{1, r}\left(\mathbb{R}^{d}\right)$, then the compactness of the embedding $\mathcal{W}^{2, d}\left(B_{R}\right) \hookrightarrow \mathcal{C}^{1, r}\left(\bar{B}_{R}\right)$ together with elliptic regularity 29 , Theorem 9.19] implies that solutions are in $\mathcal{C}^{2}\left(\mathbb{R}^{d}\right)$, a fact which we use often. 


\section{Proofs of the results on the multiplicative Poisson equation}

In this section we establish basic properties of the MPE through lemmas that lead to the proof of Theorems 1.5 1.8. Throughout the rest of the section $f: \mathbb{R}^{d} \rightarrow \mathbb{R}$ is a locally bounded measurable function, and $\mathcal{L}$ is as defined in (1.13). Also, as mentioned earlier, we assume that for the sde in (1.12), $\sigma$ and $b$ satisfy (A2)-(A3), $\sigma$ is locally Lipschitz (as in (A1)), and $b$ is measurable.

\subsection{Some basic results on the MPE}

Some well known properties of the MPE are summarized in the following lemma.

Lemma 2.1. Let $f$ be near-monotone relative to $\Lambda$, and $(\Psi, \Lambda) \in \mathcal{W}_{\text {loc }}^{2, p}\left(\mathbb{R}^{d}\right) \times \mathbb{R}, p \geq 1$, be a nonnegative solution to (1.15) satisfying $\Psi(0)>0$. Then the following are equivalent:

(a) The diffusion (1.12) is recurrent.

(b) $\inf _{\mathbb{R}^{d}} \Psi>0$.

(c) $\min _{\mathbb{R}^{d}} \Psi=\min _{\mathcal{K}} \Psi$, with $\mathcal{K}$ as in Definition 1.1.

(d) The function $\Psi$ is inf-compact.

(e) The diffusion (1.12) is geometrically ergodic, i.e., it is positive recurrent with invariant probability measure $\mu$, and there exist positive constants $\kappa$ and $\beta$, such that if $g: \mathbb{R}^{d} \rightarrow \mathbb{R}$ is any locally bounded measurable function satisfying $\|g\|_{\Psi}<\infty$, it holds that

$$
\left|\mathbb{E}_{x}\left[g\left(X_{t}\right)\right]-\mu(g)\right| \leq \kappa \mathrm{e}^{-\beta t}\|g\|_{\Psi}(1+\Psi(x)) \quad \forall t>0 .
$$

(f) $\Lambda_{x}(f) \leq \Lambda$ for all $x \in \mathbb{R}^{d}$.

(g) $\Lambda_{x}(f) \leq \Lambda$ for some $x \in \mathbb{R}^{d}$.

Proof. Let $\mathcal{B}$ be a bounded ball and $\delta>0$ a constant, such that $f-\Lambda>\delta$ in $\mathcal{B}^{c}$. Then with $\breve{\tau} \equiv \tau\left(\mathcal{B}^{c}\right)$ we have

$$
\begin{aligned}
\Psi(x) & \geq \mathbb{E}_{x}\left[\mathrm{e}^{\delta \breve{\tau}} \Psi\left(X_{\breve{\tau}}\right) \mathbb{1}\{\breve{\tau}<\infty\}\right] \\
& \geq\left(\inf _{\partial \mathcal{B}} \Psi\right) \mathbb{E}_{x}\left[\mathrm{e}^{\delta \breve{\tau}} \mathbb{1}\{\breve{\tau}<\infty\}\right] \quad \forall x \in \overline{\mathcal{B}}^{c} .
\end{aligned}
$$

If (a) holds, then since $\inf _{\mathcal{B}} \Psi>0$ by the Harnack inequality, and $x \mapsto \mathbb{E}_{x}\left[\mathrm{e}^{\delta \breve{\tau}} \mathbb{1}\{\breve{\tau}<\infty\}\right]$ is inf-compact, by Assumption (A2), then both (c) and (d) follow, and of course either (c) or (d) imply (b). Thus to complete the proof it suffices to show that (b) implies (e) and (f) and that (g) implies (a).

Suppose $\inf _{\mathbb{R}^{d}} \Psi>0$. Then since $\mathcal{L} \Psi<-\delta \Psi$ on $\mathcal{B}^{c}$, (e) follows (see [23, 28]). Also, by (1.15) and Fatou's lemma we have

$$
\begin{aligned}
\Psi(x) & \geq \mathbb{E}_{x}\left[\mathrm{e}^{\int_{0}^{T}\left[f\left(X_{t}\right)-\Lambda\right] \mathrm{d} t} \Psi\left(X_{T}\right)\right] \\
& \geq\left(\inf _{\mathbb{R}^{d}} \Psi\right) \mathbb{E}_{x}\left[\mathrm{e}^{\int_{0}^{T}\left[f\left(X_{t}\right)-\Lambda\right] \mathrm{d} t}\right],
\end{aligned}
$$

and (f) follows by taking log, dividing by $T$ and letting $T \rightarrow \infty$.

It is obvious that $(\mathrm{f}) \Rightarrow(\mathrm{g})$. We next show that $(\mathrm{g}) \Rightarrow(\mathrm{a})$, and this completes the proof. Using Jensen's inequality we have

$$
\limsup _{T \rightarrow \infty} \frac{1}{T} \int_{0}^{T} \mathbb{E}_{x}\left[f\left(X_{t}\right)\right] \mathrm{d} t \leq \Lambda_{x}(f) \leq \Lambda .
$$

A standard argument then shows that any limit point of ergodic occupation measures along some sequence $T_{n} \rightarrow \infty$ in $\mathcal{P}\left(\mathbb{R}^{d} \cup\{\infty\}\right)$, i.e., the set of probability measures on the one point compactification of $\mathbb{R}^{d}$, takes the form $\rho \mu+(1-\rho) \delta_{\infty}$, with $\rho \in(0,1)$ and that $\mu$ is an invariant probability measure of (1.12) (see Lemma 3.4.6 and Theorem 3.4.7 in [2]). This of course implies that (1.12) is positive recurrent. 
The following lemma summarizes some results from [7, 8, 46] on eigenvalues of the Dirichlet problem for the operator $\mathcal{L}$. Recall that subsets $A$ and $B$ of $\mathbb{R}^{d}$ we write $A \Subset B$ if $\bar{A} \subset B$.

Lemma 2.2. For each $r \in(0, \infty)$ there exists a unique pair $\left(\widehat{\Psi}_{r}, \hat{\lambda}_{r}\right) \in\left(\mathcal{W}^{2, p}\left(B_{r}\right) \cap \mathcal{C}\left(\bar{B}_{r}\right)\right) \times \mathbb{R}$, for any $p>d$, satisfying $\widehat{\Psi}_{r}>0$ on $B_{r}, \widehat{\Psi}_{r}=0$ on $\partial B_{r}$, and $\widehat{\Psi}_{r}(0)=1$, which solves

$$
\mathcal{L} \widehat{\Psi}_{r}(x)+f(x) \widehat{\Psi}_{r}(x)=\hat{\lambda}_{r} \widehat{\Psi}_{r}(x) \quad \text { a.e. } x \in B_{r} .
$$

Moreover, solution has the following properties:

(a) The map $r \mapsto \hat{\lambda}_{r}$ is continuous and strictly increasing.

(b) In its dependence on the function $f, \hat{\lambda}_{r}$ is nondecreasing, convex, and Lipschitz continuous (with respect to the $L^{\infty}$ norm) with Lipschitz constant 1 . In addition, if $f \supsetneqq f^{\prime}$ then $\hat{\lambda}_{r}(f)<\hat{\lambda}_{r}\left(f^{\prime}\right)$.

Proof. Existence and uniqueness of the solution follow by [46, Theorem 1.1] (see also [8]). Part (a) follows by [7, Theorem 1.10], and (iii)-(iv) of [7, Proposition 2.3] while part [(b)] follows by [8, Proposition 2.1].

We refer to the pair $\left(\widehat{\Psi}_{r}, \hat{\lambda}_{r}\right)$ in Lemma 2.2 as the Dirichlet eigensolution of the MPE on $B_{r}$. We also call $\widehat{\Psi}_{r}$, and $\hat{\lambda}_{r}$, the Dirichlet eigenfunction and eigenvalue on $B_{r}$, respectively.

Lemma 2.3. Let $\left(\widehat{\Psi}_{r}, \hat{\lambda}_{r}\right)$ be as in Lemma 2.2. Then

(i) $\hat{\lambda}_{r}<\inf _{x \in B_{r}} \Lambda_{x}(f)$ for all $r \in(0, \infty)$.

(ii) If $\Lambda(f)<\infty$, then every sequence $\left\{r_{n}\right\}$, with $r_{n} \rightarrow \infty$ contains a subsequence also denoted as $\left\{r_{n}\right\}$, along which $\left(\widehat{\Psi}_{r_{n}}, \hat{\lambda}_{r_{n}}\right)$ in (2.2) converge to some $(\widehat{\Psi}, \hat{\lambda}) \in \mathcal{W}_{\text {loc }}^{2, p}\left(\mathbb{R}^{d}\right) \times \mathbb{R}, p>d$, which solves the MPE (1.15).

(iii) If the diffusion in (1.12) is recurrent and $f$ is near-monotone relative to $\Lambda(f)$, then $\Lambda_{x}(f)=\Lambda(f)$ for all $x \in \mathbb{R}^{d}$, and $\hat{\lambda}_{r} \nearrow \Lambda(f)$ as $r \rightarrow \infty$.

PROOF. Since $\widehat{\Psi}_{r}=0$ on $\tau_{r}=\tau\left(B_{r}^{c}\right)$, applying Itô's formula to (2.2) we obtain

$$
\begin{aligned}
\widehat{\Psi}_{r}(x) & =\mathbb{E}_{x}\left[\mathrm{e}^{\int_{0}^{T}\left[f\left(X_{t}\right)-\hat{\lambda}_{r}\right] \mathrm{d} t} \widehat{\Psi}_{r}\left(X_{T}\right) \mathbb{1}_{\left\{T \leq \tau_{r}\right\}}\right] \\
& \leq\left\|\widehat{\Psi}_{r}\right\|_{\infty, B_{r}} \mathbb{E}_{x}\left[\mathrm{e}^{\left.\int_{0}^{T}\left[f\left(X_{t}\right)-\hat{\lambda}_{r}\right] \mathrm{d} t\right] \quad \forall(T, x) \in \mathbb{R}_{+} \times B_{r} .}\right.
\end{aligned}
$$

Therefore, we have

$$
\Lambda_{x}(f)=\limsup _{T \rightarrow \infty} \frac{1}{T} \log \mathbb{E}_{x}\left[\mathrm{e}^{\int_{0}^{T} f\left(X_{t}\right) \mathrm{d} t}\right] \geq \hat{\lambda}_{r} \quad \forall x \in B_{r},
$$

which proves part (i). Part (ii) is as in [11, Lemma 2.1].

Suppose $\hat{\lambda}_{r_{n}} \rightarrow \hat{\lambda}$ along some sequence $r_{n} \nearrow \infty$. By part (ii) there exists a subsequence also denoted as $\left\{r_{n}\right\}$ along which $\widehat{\Psi}_{r_{n}} \rightarrow \widehat{\Psi} \in \mathcal{W}_{\text {loc }}^{2, p}\left(\mathbb{R}^{d}\right) \times \mathbb{R}, p>d$, and the pair $(\widehat{\Psi}, \hat{\lambda})$ solves the MPE (1.15). By Lemma 2.1(f) we have $\hat{\lambda} \geq \Lambda_{x}(f)$ for all $x \in \mathbb{R}^{d}$, which combined with part (i) results in equality.

By Lemma 2.1. (H1) implies that (1.12) is positive recurrent. Also without loss of generality we may assume that $\Lambda(f)>0$, otherwise we translate $f$ by a constant to attain this. Lemma 2.3 shows that there exists a positive solution $\Psi \in \mathcal{W}_{\text {loc }}^{2, p}\left(\mathbb{R}^{d}\right), p>d$, to

$$
\mathcal{L} \Psi(x)+f(x) \Psi(x)=\Lambda(f) \Psi(x) \quad \text { a.e. } x \in \mathbb{R}^{d} .
$$

Then, necessarily $\inf _{\mathbb{R}^{d}} \Psi>0$ by Lemma 2.1. Unless stated otherwise, we use the symbol $\Psi$ to denote a positive solution of (2.3).

We continue with the proof of Theorem 1.4 
Proof of Theorem 1.4. Let $\epsilon>0$ and $\mathcal{K}_{\epsilon}$ a compact set in $\mathbb{R}^{d}$ such that $\operatorname{essinf}_{\mathcal{K}_{\epsilon}^{c}}(f-\Lambda(f)-\epsilon) \geq 0$. If a positive function $\varphi \in \mathcal{W}_{\text {loc }}^{2, d}\left(\mathbb{R}^{d}\right)$, satisfies $\mathcal{L} \varphi+(f-\lambda) \varphi \leq 0$ for some $\lambda \leq \Lambda(f)+\epsilon$, then by the proof of Lemma 2.1(d), we have $\inf _{\mathbb{R}^{d}} \varphi>0$. On the other hand, if $\mathcal{L} \varphi+(f-\lambda) \varphi \leq 0$ and $\inf _{\mathbb{R}^{d}} \varphi>0$, then as in the proof of Lemma 2.1(f) we obtain $\Lambda_{x}(f) \leq \lambda$ for all $x \in \mathbb{R}^{d}$. Thus $\Lambda(f) \leq \hat{\Lambda}(f)$, which implies that $\Lambda(f) \leq \hat{\Lambda}(f) \leq \hat{\hat{\Lambda}}(f)$. However, $\Psi$ satisfies $\mathcal{L} \Psi+(f-\Lambda(f)) \Psi \leq 0$ and therefore $\Lambda(f) \geq \hat{\hat{\Lambda}}(f)$, which results in equality. The proof is complete.

\subsection{Results concerning Theorem 1.5}

Recall the definitions of the ground state diffusion in (1.17), the operator in (1.18), and the ground state semigroup in (1.20). The operators $\mathcal{L}$ and $\mathcal{L}^{*}$ are linked via the following useful identity. If $\Psi$ is a positive solution of (2.3) and $\tilde{\Psi}$ is a positive solution of

$$
\mathcal{L} \tilde{\Psi}(x)+\tilde{f}(x) \tilde{\Psi}(x)=\Lambda(\tilde{f}) \tilde{\Psi}(x) \quad \text { a.e. } x \in \mathbb{R}^{d},
$$

then

$$
\mathcal{L}^{*}\left(\tilde{\Psi} \Psi^{-1}\right)=(\Lambda(\tilde{f})-\Lambda(f)+f-\tilde{f}) \tilde{\Psi} \Psi^{-1} .
$$

Throughout this section we assume (H1). Recall the definition in (1.19). We start with the following lemma.

Lemma 2.4. Let

$$
\mathcal{M}_{t}:=\exp \left(\int_{0}^{t}\left\langle\sigma^{\top}\left(X_{s}\right) \nabla \psi\left(X_{s}\right), \mathrm{d} W_{s}\right\rangle \mathrm{d} s-\frac{1}{2} \int_{0}^{t}\langle\nabla \psi, a \nabla \psi\rangle\left(X_{s}\right) \mathrm{d} s\right) .
$$

Then $\left(\mathcal{M}_{t}, \mathfrak{F}_{t}^{X}\right)$ is a martingale if and only if

$$
\mathrm{e}^{-\Lambda(f) t} P_{t}^{f} \Psi(x)=\Psi(x) \quad \forall(t, x) \in \mathbb{R}_{+} \times \mathbb{R}^{d} .
$$

Proof. Recall that $\psi=\log \Psi$. We use the first exit times from $B_{n}$, i.e., $\tau_{n}$ as localization times. Since the drift of (1.12) satisfies (A2), it is well-known that $\tau_{n} \rightarrow \infty$ as $n \rightarrow \infty \mathbb{P}_{x}$-a.s. Applying the Itô-Krylov formula [38, p. 122] to (1.12) we obtain

$$
\begin{aligned}
\psi\left(X_{t \wedge \tau_{n}}\right)-\psi(x) & =\int_{0}^{t \wedge \tau_{n}} \mathcal{L}\left(X_{s}\right) \mathrm{d} s+\int_{0}^{t \wedge \tau_{n}}\left\langle\nabla \psi\left(X_{s}\right), \sigma\left(X_{s}\right) \mathrm{d} W_{s}\right\rangle \\
= & \int_{0}^{t \wedge \tau_{n}}\left(\Lambda(f)-f\left(X_{s}\right)-\frac{1}{2}\langle\nabla \psi, a \nabla \psi\rangle\left(X_{s}\right)\right) \mathrm{d} s \\
& +\int_{0}^{t \wedge \tau_{n}}\left\langle\nabla \psi\left(X_{s}\right), \sigma\left(X_{s}\right) \mathrm{d} W_{s}\right\rangle .
\end{aligned}
$$

By (2.6) we have

$$
\begin{aligned}
\mathbb{E}_{x}\left[\mathrm{e}^{\int_{0}^{t \wedge \tau_{n}}\left[f\left(X_{s}\right)-\Lambda(f)\right] \mathrm{d} s} g\left(X_{t \wedge \tau_{n}}\right)\right] \\
=\mathbb{E}_{x}\left[g ( X _ { t \wedge \tau _ { n } } ) \operatorname { e x p } \left(-\psi\left(X_{t \wedge \tau_{n}}\right)+\psi(x)\right.\right. \\
\left.\left.\quad+\int_{0}^{t \wedge \tau_{n}}\left\langle\nabla \psi\left(X_{s}\right), \sigma\left(X_{s}\right) \mathrm{d} W_{s}\right\rangle-\int_{0}^{t \wedge \tau_{n}} \frac{1}{2}\langle\nabla \psi, a \nabla \psi\rangle\left(X_{s}\right) \mathrm{d} s\right)\right] \\
=\Psi(x) \mathbb{E}_{x}\left[g\left(X_{t \wedge \tau_{n}}\right) \Psi^{-1}\left(X_{t \wedge \tau_{n}}\right) \mathcal{M}_{t \wedge \tau_{n}}\right] .
\end{aligned}
$$


Thus choosing an increasing sequence $g_{m} \in \mathcal{C}_{b}\left(\mathbb{R}^{d}\right)$ which converges to $\Psi$, uniformly on compact sets, we obtain

$$
\mathbb{E}_{x}\left[\mathrm{e}^{\int_{0}^{t \wedge \tau_{n}}\left[f\left(X_{s}\right)-\Lambda(f)\right] \mathrm{d} s} \Psi\left(X_{t \wedge \tau_{n}}\right)\right]=\Psi(x) \mathbb{E}_{x}\left[\mathcal{M}_{t \wedge \tau_{n}}\right]
$$

by monotone convergence. It follows that $\mathbb{E}_{x}\left[\mathcal{M}_{t \wedge \tau_{n}}\right]=1$, so that $\left(\mathcal{M}_{t}, \mathfrak{F}_{t}^{X}\right)$ is a local martingale.

Next consider $g \in \mathcal{C}_{c}\left(\mathbb{R}^{d}\right)$. By (2.7) we obtain

$$
\mathbb{E}_{x}\left[\mathrm{e}^{\int_{0}^{t}\left[f\left(X_{s}\right)-\Lambda(f)\right] \mathrm{d} s} g\left(X_{t}\right) \Psi\left(X_{t}\right) \mathbb{1}_{\left\{t \leq \tau_{n}\right\}}\right]=\Psi(x) \mathbb{E}_{x}\left[g\left(X_{t}\right) \mathcal{M}_{t} \mathbb{1}_{\left\{t \leq \tau_{n}\right\}}\right]
$$

for all sufficiently large $n$, and therefore also

$$
\mathbb{E}_{x}\left[\mathrm{e}^{\int_{0}^{t}\left[f\left(X_{s}\right)-\Lambda(f)\right] \mathrm{d} s} g\left(X_{t}\right) \Psi\left(X_{t}\right)\right]=\Psi(x) \mathbb{E}_{x}\left[g\left(X_{t}\right) \mathcal{M}_{t}\right]
$$

by monotone convergence. Thus evaluating (2.8) on some increasing sequence $g_{n} \in \mathcal{C}_{c}\left(\mathbb{R}^{d}\right)$ which converges to 1 , uniformly on compact sets, and taking limits as $n \rightarrow \infty$, using again monotone convergence and (2.5), we obtain

$$
\Psi(x)=\mathbb{E}_{x}\left[\mathrm{e}^{\int_{0}^{t}\left[f\left(X_{s}\right)-\Lambda(f)\right] \mathrm{d} s} \Psi\left(X_{t}\right)\right]=\Psi(x) \mathbb{E}_{x}\left[\mathcal{M}_{t}\right] .
$$

If (2.5) holds then $\mathbb{E}\left[\mathcal{M}_{t}\right]=1$ by (2.9). Thus $\left(\mathcal{M}_{t}, \mathfrak{F}_{t}^{X}\right)$ is a martingale. Conversely, if $\mathbb{E}\left[\mathcal{M}_{t}\right]=1$, then (2.9) implies (2.5). This completes the proof.

Lemma 2.5. The sde in (1.17) has a unique strong solution $X^{*}$ which exists for all $t>0$ if and only if $\left(\mathcal{M}_{t}, \mathfrak{F}_{t}^{X}\right)$ is a martingale.

Proof. By (2.7) we have

$$
\begin{aligned}
\mathbb{E}_{x}\left[\mathrm{e}^{\int_{0}^{t \wedge \tau_{n}}\left[f\left(X_{s}\right)-\Lambda(f)\right] \mathrm{d} s} g\left(X_{t \wedge \tau_{n}}\right)\right] & =\Psi(x) \mathbb{E}_{x}\left[g\left(X_{t \wedge \tau_{n}}\right) \Psi^{-1}\left(X_{t \wedge \tau_{n}}\right) \mathcal{M}_{t \wedge \tau_{n}}\right] \\
& =\mathbb{E}_{x}^{*}\left[g\left(X_{t \wedge \tau_{n}}^{*}\right) \exp \left(-\psi\left(X_{t \wedge \tau_{n}}^{*}\right)+\psi(x)\right)\right],
\end{aligned}
$$

where in the last line we use Cameron-Martin-Girsanov theorem [40, p. 225].

By the second equality in (2.10) we have

$$
\mathbb{E}_{x}\left[g\left(X_{t \wedge \tau_{n}}\right) \Psi^{-1}\left(X_{t \wedge \tau_{n}}\right) \mathcal{M}_{t \wedge \tau_{n}}\right]=\mathbb{E}_{x}^{*}\left[g\left(X_{t \wedge \tau_{n}}^{*}\right) \Psi^{-1}\left(X_{t \wedge \tau_{n}}^{*}\right)\right]
$$

for any $g \in \mathcal{C}_{b}\left(\mathbb{R}^{d}\right)$. Let $h: \mathbb{R}^{d} \rightarrow[0,1]$ be a continuous function which is equal to 1 on $B_{n-1}$, and vanishes on $B_{n}^{c}$. Evaluating (2.11) on $g=h \Psi$ we obtain $\mathbb{P}_{x}^{*}\left(t<\tau_{n}\right) \geq \mathbb{E}_{x}\left[\mathcal{M}_{t} \mathbb{1}_{\left\{t \leq \tau_{n-1}\right\}}\right]$ by (2.11). Therefore, if $\left(\mathcal{M}_{t}, \mathfrak{F}_{t}^{X}\right)$ is a martingale, then with $\tau_{\infty}:=\lim _{n \rightarrow \infty} \tau_{n}$, we have $\mathbb{P}_{x}^{*}\left(t<\tau_{\infty}\right)=\mathbb{E}_{x}\left[\mathcal{M}_{t}\right]=1$. Thus

$$
\mathbb{P}_{x}^{*}\left(\tau_{\infty}=\infty\right)=\lim _{t \rightarrow \infty} \mathbb{P}_{x}^{*}\left(t<\tau_{\infty}\right)=1
$$

which shows that the diffusion is regular.

The same argument shows that $\mathbb{P}_{x}^{*}\left(t<\tau_{n-1}\right) \leq \mathbb{E}_{x}\left[\mathcal{M}_{t} \mathbb{1}_{\left\{t \leq \tau_{n}\right\}}\right]$, from which it follows that if the $X^{*}$ process is regular, then $\left(\mathcal{M}_{t}, \mathfrak{F}_{t}^{X}\right)$ is a martingale. This completes the proof.

Lemma 2.6. If process $X^{*}$ in (1.17) is recurrent, then, for any $r>0$, we have

$$
\Psi(x)=\mathbb{E}_{x}\left[\mathrm{e}^{\int_{0}^{\breve{r}_{r}}\left[f\left(X_{s}\right)-\Lambda(f)\right] \mathrm{d} s} \Psi\left(X_{\breve{\tau}_{r}}\right)\right], \quad \text { for } x \in B_{r}^{c} .
$$

Conversely, if (2.12) holds for some $r>0$ then the process $X^{*}$ is recurrent. 
Proof. Let $r>\epsilon>0$, and $h: \mathbb{R}^{d} \rightarrow[0,1]$ be a continuous function which equals 1 on $\bar{B}_{r}$ and vanishes on $B_{r+\epsilon}^{c}$. Using (2.10) with $g=h \Psi$ and localized at $\breve{\tau}_{r} \wedge t \wedge \tau_{n}$, and then taking limits as $\epsilon \searrow 0$, we obtain

$$
\mathbb{E}_{x}\left[\mathrm{e}^{\int_{0}^{t \wedge \breve{\tau}_{r}}\left[f\left(X_{s}\right)-\Lambda(f)\right] \mathrm{d} s} \Psi\left(X_{t \wedge \breve{\tau}_{r}}\right) \mathbb{1}_{\bar{B}_{r}}\left(X_{t \wedge \breve{\tau}_{r}}\right) \mathbb{1}_{\left\{t \wedge \breve{\tau}_{r}<\tau_{n}\right\}}\right]=\Psi(x) \mathbb{P}_{x}^{*}\left(\breve{\tau}_{r} \leq t \wedge \tau_{n}\right) .
$$

By Fatou's lemma we have

$$
\mathbb{E}_{x}\left[\mathrm{e}^{\int_{0}^{\breve{t}_{r}}\left[f\left(X_{s}\right)-\Lambda(f)\right] \mathrm{d} s} \Psi\left(X_{\breve{\tau}_{r}}\right)\right] \leq \Psi(x),
$$

and therefore, taking limits in (2.13) first as $t \rightarrow \infty$, and then as $n \rightarrow \infty$, we obtain

$$
\mathbb{E}_{x}\left[\mathrm{e}^{\int_{0}^{\breve{\tau}_{r}}\left[f\left(X_{s}\right)-\Lambda(f)\right] \mathrm{d} s} \Psi\left(X_{\breve{\tau}_{r}}\right)\right]=\Psi(x) \mathbb{P}_{x}^{*}\left(\breve{\tau}_{r}<\tau_{\infty}\right) .
$$

Note that for fixed $n$ one can justify the limit as $t \rightarrow \infty$ of the term on the left hand side of (2.13) by dominated convergence. If $X^{*}$ is recurrent (and regular) then $\mathbb{P}_{x}^{*}\left(\tau_{\infty}=\infty\right)=1$, and $\mathbb{P}_{x}^{*}\left(\breve{\tau}_{r}<\infty\right)=1$ for any $r>0$, and thus (2.12) follows by (2.14).

Now suppose that (2.12) holds for some $r>0$. Then $\mathbb{P}_{x}^{*}\left(\breve{\tau}_{r}<\tau_{\infty}\right)=1$. We claim that this implies that the process is regular, i.e., $\mathbb{P}_{x}^{*}\left(\tau_{\infty}=\infty\right)=1$. To prove the claim, let $\mathfrak{s}_{0}=0$, and for $k=0,1, \ldots$ define inductively an increasing sequence of stopping times by

$$
\begin{aligned}
& \mathfrak{s}_{2 k+1}:=\inf \left\{t>\mathfrak{s}_{2 k}: X_{t}^{*} \in B_{2 r}^{c}\right\}, \\
& \mathfrak{s}_{2 k+2}:=\inf \left\{t>\mathfrak{s}_{2 k+1}: X_{t}^{*} \in B_{r}\right\} .
\end{aligned}
$$

It is clear that $\mathbb{P}_{x}^{*}\left(\breve{\tau}_{r}<\tau_{\infty}\right)=1$ implies that $\mathbb{P}_{x}^{*}\left(\mathfrak{s}_{k}<\tau_{\infty}\right)=1$. It follows that $\mathfrak{s}_{n} \nearrow \infty$, $\mathbb{P}_{x}^{*}$-a.s., by a standard argument used in the proof of [2, Lemma 2.6.6]. This proves the claim. Since the coefficients of $\mathcal{L}^{*}$ are locally bounded, Harnack's theorem applies, and thus a classical argument due to Hasminskii shows that $\mathbb{P}_{x}^{*}\left(\breve{\tau}_{r}<\infty\right)=1$ for some $r>0$ implies that the same holds for all $r>0$ [31, Lemma 2.1, p. 111]. This completes the proof.

We have the following continuity property (compare with $\mathbf{7}$, Proposition 9.2]), even though it is not used in the proof of the main results.

Lemma 2.7. Suppose $f_{n} \in L_{\mathrm{loc}}^{\infty}\left(\mathbb{R}^{d}\right)$ is a sequence of nonnegative functions converging to $f \in L_{\mathrm{loc}}^{\infty}\left(\mathbb{R}^{d}\right)$, such that

1. $\Lambda\left(f_{n}\right) \leq \Lambda(f)$ for each $n \in \mathbb{N}$.

2. For some $\epsilon>0$ the set $D_{\epsilon}:=\cup_{n \in \mathbb{N}}\left\{x: f_{n}(x)<\Lambda(f)+\epsilon\right\}$ is bounded.

Then $\Lambda\left(f_{n}\right) \rightarrow \Lambda(f)$ as $n \rightarrow \infty$.

Proof. Suppose not. Then we have $\liminf _{n \rightarrow \infty} \Lambda\left(f_{n}\right)=\bar{\Lambda}<\Lambda(f)$. Let $\Psi_{n} \in \mathcal{W}_{\text {loc }}^{2, p}, p>d$, solutions to the Poisson equation

$$
\mathcal{L} \Psi_{n}+f_{n} \Psi_{n}=\Lambda\left(f_{n}\right) \Psi_{n},
$$

satisfying $\Psi_{n}(0)=1$. Then $\inf _{\mathbb{R}^{d}} \Psi_{n}=\inf _{\bar{D}_{\epsilon}} \Psi_{n}$ by (2). Let $\bar{\Psi}$ be any limit point of $\Psi_{n}$ as $n \rightarrow \infty$, along some subsequence along which $\Lambda\left(f_{n}\right)$ converges to $\bar{\Lambda}$. Then we obtain

$$
\mathcal{L} \bar{\Psi}+f \bar{\Psi}=\bar{\Lambda} \bar{\Psi},
$$

and $\bar{\Psi}$ satisfies $\inf _{\mathbb{R}^{d}} \bar{\Psi}=\inf _{\bar{K}_{\epsilon}} \bar{\Psi}$. Then by Lemma 2.1 (f) we have $\Lambda(f) \leq \bar{\Lambda}$ which contradicts the original hypothesis.

Theorem 2.8. Suppose that for some $\hat{x} \in \mathbb{R}^{d}$ we have

$$
\Gamma(\hat{x}):=\int_{0}^{\infty} \mathbb{E}_{\hat{x}}\left[\mathrm{e}^{\int_{0}^{t}\left[f\left(X_{s}\right)-\Lambda(f)\right] \mathrm{d} s}\right] \mathrm{d} t=\infty .
$$


Then (2.3) has a unique positive solution $\Psi \in \mathcal{W}_{\mathrm{loc}}^{2, d}\left(\mathbb{R}^{d}\right)$, satisfying $\Psi(0)=1$. In addition, $\Psi$ satisfies

$$
\Psi(x)=\mathbb{E}_{x}\left[\mathrm{e}^{\int_{0}^{\tau\left(\mathcal{B}^{c}\right)}\left[f\left(X_{s}\right)-\Lambda(f)\right] \mathrm{d} s} \Psi\left(X_{\tau\left(\mathcal{B}^{c}\right)}\right)\right] \quad \forall x \in \mathcal{B}^{c}
$$

for some ball $\mathcal{B}$.

Proof. Let $\theta>0$ be any positive constant such that $f$ is near monotone relative to $\Lambda(f)+2 \theta$, and fix some $\alpha \in(0, \theta)$. In order to simplify the notation we define

$$
F_{\alpha}(x):=f(x)-\Lambda(f)-\alpha, \quad \text { and } \quad F_{0}(x):=f(x)-\Lambda(f),
$$

and

$$
\Gamma_{\alpha}(x):=\int_{0}^{\infty} \mathbb{E}_{x}\left[\mathrm{e}^{\int_{0}^{t} F_{\alpha}\left(X_{s}\right) \mathrm{d} s}\right] \mathrm{d} t .
$$

Without loss of generality we assume $\hat{x}=0$. Moreover, $\Gamma_{\alpha}(0)$ is finite follows from the fact that

$$
\begin{aligned}
\mathbb{E}_{x}\left[\mathrm{e}^{\int_{0}^{t} F_{\alpha}\left(X_{s}\right) \mathrm{d} s}\right] & \leq \mathrm{e}^{-\alpha t}\left(\inf _{\mathbb{R}^{d}} \Psi\right)^{-1} \mathbb{E}_{x}\left[\mathrm{e}^{\int_{0}^{t} F_{0}\left(X_{s}\right) \mathrm{d} s} \Psi\left(X_{t}\right)\right] \\
& \leq \mathrm{e}^{-\alpha t}\left(\inf _{\mathbb{R}^{d}} \Psi\right)^{-1} \Psi(x) \quad \forall t>0 .
\end{aligned}
$$

Recall the eigenvalues $\left\{\hat{\lambda}_{n}\right\}$ in Lemma 2.2. Since $\hat{\lambda}_{n}<\Lambda(f)$, the principal eigenvalue of the operator $-\mathcal{L}-F_{\alpha}$ on every $B_{n}$ is positive. Thus by Proposition 6.2 and Theorem 6.1 in [8], for any $n \in \mathbb{N}$, the Dirichlet problem

$$
\mathcal{L} \varphi_{\alpha, n}(x)+F_{\alpha}(x) \varphi_{\alpha, n}(x)=-\Gamma_{\alpha}^{-1}(0) \quad \text { a.e. } x \in B_{n}, \quad \varphi_{\alpha, n}=0 \text { on } \partial B_{n},
$$

has a unique solution $\varphi_{\alpha, n} \in \mathcal{W}_{\text {loc }}^{2, p}\left(B_{n}\right) \cap \mathcal{C}\left(\bar{B}_{n}\right)$, for any $p \geq 1$. In addition, by the refined maximum principle in [8, Theorem 1.1] $\varphi_{\alpha, n}$ is nonnegative. Since $\Gamma_{\alpha}^{-1}(0)>0$, then $\varphi_{\alpha, n}$ cannot be identically equal to 0 . Thus writing (2.17) as

$$
\mathcal{L} \varphi_{\alpha, n}-F_{\alpha}^{-} \varphi_{\alpha, n}=-F_{\alpha}^{+} \varphi_{\alpha, n}-\Gamma_{\alpha}^{-1}(0),
$$

it follows by the strong maximum principle that $\varphi_{\alpha, n}>0$ in $B_{n}$.

By Itô's formula we obtain from (2.17) that

$$
\begin{aligned}
\varphi_{\alpha, n}(x) & =\mathbb{E}_{x}\left[\mathrm{e}^{\int_{0}^{T \wedge \tau_{n}} F_{\alpha}\left(X_{s}\right) \mathrm{d} s} \varphi_{\alpha, n}\left(X_{T \wedge \tau_{n}}\right)\right]+\Gamma_{\alpha}^{-1}(0) \mathbb{E}_{x}\left[\int_{0}^{T \wedge \tau_{n}} \mathrm{e}^{\int_{0}^{t} F_{\alpha}\left(X_{s}\right) \mathrm{d} s} \mathrm{~d} t\right] \\
& =\mathbb{E}_{x}\left[\mathrm{e}^{\int_{0}^{T} F_{\alpha}\left(X_{s}\right) \mathrm{d} s} \varphi_{\alpha, n}\left(X_{T}\right) \mathbb{1}_{\left\{T \leq \tau_{n}\right\}}\right]+\Gamma_{\alpha}^{-1}(0) \mathbb{E}_{x}\left[\int_{0}^{T \wedge \tau_{n}} \mathrm{e}^{\int_{0}^{t} F_{\alpha}\left(X_{s}\right) \mathrm{d} s} \mathrm{~d} t\right]
\end{aligned}
$$

for all $(T, x) \in \mathbb{R}_{+} \times B_{n}$, where we use the property that $\varphi_{\alpha, n}=0$ on $\partial B_{n}$. Let $\Psi$ be any positive solution of (2.3), satisfying $\Psi(0)=1$. Writing (2.3) as

$$
\mathcal{L} \Psi(x)+F_{\alpha}(x) \Psi(x)=-\alpha \Psi(x) \quad \text { a.e. } x \in \mathbb{R}^{d},
$$

and using Itô's formula and Fatou's lemma we obtain

$$
\Psi(x) \geq \mathbb{E}_{x}\left[\mathrm{e}^{\int_{0}^{\tau_{n} n} F_{\alpha}\left(X_{s}\right) \mathrm{d} s} \Psi\left(X_{\tau_{n}}\right)\right]+\alpha \mathbb{E}_{x}\left[\int_{0}^{\tau_{n}} \mathrm{e}^{\int_{0}^{t} F_{\alpha}\left(X_{s}\right) \mathrm{d} s} \Psi\left(X_{t}\right) \mathrm{d} t\right],
$$

for any $\alpha \geq 0$. By Itô's formula and Fatou's lemma, we have from (2.3) that

$$
\mathbb{E}_{x}\left[\mathrm{e}^{\int_{0}^{t} F_{0}\left(X_{s}\right) \mathrm{d} s}\right] \leq\left(\inf _{\mathbb{R}^{d}} \Psi\right)^{-1} \Psi(x) \quad \forall t>0, \quad \forall x \in \mathbb{R}^{d} .
$$


Therefore,

$$
\mathbb{E}_{x}\left[\mathrm{e}^{\int_{0}^{T} F_{\alpha}\left(X_{s}\right) \mathrm{d} s} \varphi_{\alpha, n}\left(X_{T}\right) \mathbb{1}_{\left\{T \leq \tau_{n}\right\}}\right] \leq \mathrm{e}^{-\alpha T}\left(\sup _{B_{n}} \varphi_{\alpha, n}\right) \mathbb{E}_{x}\left[\mathrm{e}^{\int_{0}^{T} F_{0}\left(X_{s}\right) \mathrm{d} s} \mathbb{1}_{\left\{T \leq \tau_{n}\right\}}\right] \underset{T \rightarrow \infty}{\longrightarrow} 0 .
$$

Thus taking limits in (2.18) as $T \rightarrow \infty$, using monotone convergence for the second integral, we obtain

$$
\varphi_{\alpha, n}(x)=\Gamma_{\alpha}^{-1}(0) \mathbb{E}_{x}\left[\int_{0}^{\tau_{n}} \mathrm{e}^{\int_{0}^{t} F_{\alpha}\left(X_{s}\right) \mathrm{d} s} \mathrm{~d} t\right],
$$

which implies by (2.19) that

$$
\varphi_{\alpha, n} \leq \frac{1}{\alpha \Gamma_{\alpha}(0)}\left(\inf _{\mathbb{R}^{d}} \Psi\right) \Psi \quad \forall n \in \mathbb{N}
$$

It therefore follows by (1.31) that $\left\{\varphi_{\alpha, n}\right\}$ is relatively weakly compact in $\mathcal{W}^{2, p}\left(B_{n}\right)$, for any $p \geq 1$ and $n \in \mathbb{N}$, and as explained in Section 1.4. $\varphi_{\alpha, n}$ converges uniformly on compact sets along some sequence $n \rightarrow \infty$ to a nonnegative $\Phi_{\alpha} \in \mathcal{W}_{\text {loc }}^{2, p}\left(\mathbb{R}^{d}\right)$, for any $p \geq 1$, which solves

$$
\mathcal{L} \Phi_{\alpha}(x)+F_{\alpha}(x) \Phi_{\alpha}(x)=-\Gamma_{\alpha}^{-1}(0) \quad \text { a.e. } x \in \mathbb{R}^{d}
$$

It is clear by the strong maximum principle and since also $\Gamma_{\alpha}^{-1}(0)>0$ that $\Phi_{\alpha}>0$. By (2.18) and dominated and monotone convergence, we obtain

$$
\Phi_{\alpha}(x)=\mathbb{E}_{x}\left[\mathrm{e}^{\int_{0}^{T} F_{\alpha}\left(X_{s}\right) \mathrm{d} s} \Phi_{\alpha}\left(X_{T}\right)\right]+\Gamma_{\alpha}^{-1}(0) \mathbb{E}_{x}\left[\int_{0}^{T} \mathrm{e}^{\int_{0}^{t} F_{\alpha}\left(X_{s}\right) \mathrm{d} s} \mathrm{~d} t\right]
$$

for all $T>0$ and $x \in \mathbb{R}^{d}$, and since (2.18) holds with $T$ replaced by $\breve{\tau}_{r}$, we also have

$$
\Phi_{\alpha}(x)=\mathbb{E}_{x}\left[\mathrm{e}^{\int_{0}^{\breve{\tau}_{r}} F_{\alpha}\left(X_{s}\right) \mathrm{d} s} \Phi_{\alpha}\left(X_{\breve{\tau}_{r}}\right)\right]+\Gamma_{\alpha}^{-1}(0) \mathbb{E}_{x}\left[\int_{0}^{\breve{\tau}_{r}} \mathrm{e}^{\int_{0}^{t} F_{\alpha}\left(X_{s}\right) \mathrm{d} s} \mathrm{~d} t\right]
$$

for all $x \in B_{r}^{c}$ and $r>0$. It also follows by letting $T \rightarrow \infty$ in (2.21) that $\Phi_{\alpha}(0)=1$.

Recall the constant $\theta>0$ in the beginning of the proof, and the set $\mathcal{K}_{\theta}$ in Definition 1.1. Let $\mathcal{B}$ be a ball containing $\mathcal{K}_{\theta}$. With $\breve{\tau}=\tau\left(\mathcal{B}^{c}\right)$, and integration by parts, we obtain

$$
\begin{aligned}
\mathbb{E}_{x}\left[\int_{0}^{\breve{\tau}} \mathrm{e}^{\int_{0}^{t} F_{0}\left(X_{s}\right) \mathrm{d} s} \mathrm{~d} t\right] & \leq \frac{1}{\theta} \mathbb{E}_{x}\left[\int_{0}^{\breve{\tau}}\left[f\left(X_{t}\right)-\Lambda(f)\right] \mathrm{e}^{\int_{0}^{t}\left[f\left(X_{s}\right)-\Lambda(f)\right] \mathrm{d} s} \mathrm{~d} t\right] \\
& \leq \frac{1}{\theta} \mathbb{E}_{x}\left[\mathrm{e}^{\left.\int_{0}^{\breve{\tau}}\left[f\left(X_{s}\right)-\Lambda(f)\right] \mathrm{d} s\right]}\right. \\
& \leq\left(\theta \inf _{\partial \mathcal{B}} \Psi\right)^{-1} \Psi(x) \quad \forall x \in \mathcal{B}^{c} .
\end{aligned}
$$

It follows by 2.15) that $\Gamma_{\alpha}^{-1}(0)$ is bounded uniformly over $\alpha \in(0,1)$. Thus, the assumptions are met for the Harnack inequality for a class of superharmonic functions in [1], and we obtain by (2.20) that

$$
\Phi_{\alpha}(x) \leq C_{H}, \quad \forall x \in \overline{\mathcal{B}}, \quad \forall \alpha \in(0,1),
$$

for some constant $C_{H}$. It then follows by (2.22), (2.23), and (2.24) that the collection $\left\{\Phi_{\alpha}, \alpha \in(0,1)\right\}$ is locally bounded, and thus also relatively weakly compact in $\mathcal{W}^{2, p}\left(B_{R}\right)$ for any $p \geq 1$ and $R>0$, by (2.20). Taking limits along some sequence $\alpha \searrow 0$, we obtain a positive function $\Phi \in \mathcal{W}_{\text {loc }}^{2, d}\left(\mathbb{R}^{d}\right)$ which satisfies

$$
\mathcal{L} \Phi(x)+F_{0}(x) \Phi(x)=0 \quad \text { a.e. } x \in \mathbb{R}^{d},
$$


and $\Phi(0)=1$. By (2.15), (2.22), and (2.23) we obtain

$$
\Phi(x)=\mathbb{E}_{x}\left[\mathrm{e}^{\mathrm{\int}_{0}^{\breve{\tau}} F_{0}\left(X_{s}\right) \mathrm{d} s} \Phi\left(X_{\breve{\tau})}\right] .\right.
$$

Also by Fatou's lemma we have

$$
\mathbb{E}_{x}\left[\mathrm{e}^{\int_{0}^{\breve{r}_{r}} F_{0}\left(X_{s}\right) \mathrm{d} s} \Psi\left(X_{\breve{\tau}}\right)\right] \leq \Psi(x) \quad \forall x \in B_{r}^{c}, \quad \forall r>0 .
$$

This shows by the comparison principle (see the same argument detailed below) that $\Psi=\Phi$ and hence (2.16) holds.

We next prove uniqueness. Let $\mathcal{B}$ be a ball that contains $\mathcal{K}$ in Definition 1.1 and let $\breve{\tau}=\tau\left(\mathcal{B}^{c}\right)$. If $\tilde{\Psi}$ is any positive solution of (2.3) with $\tilde{\Psi}(0)=1$, then

$$
\tilde{\Psi}(x) \geq \mathbb{E}_{x}\left[\mathrm{e}^{\int_{0}^{\breve{c}} F_{0}\left(X_{s}\right) \mathrm{d} s} \tilde{\Psi}\left(X_{\breve{\tau}}\right)\right] \quad \forall x \in \mathcal{B}^{c}, \quad \forall r>0,
$$

by Itô's formula and Fatou's lemma. This together with (2.16) implies that

$$
\begin{aligned}
\tilde{\Psi}(x)-\Psi(x) & \geq \mathbb{E}_{x}\left[\mathrm{e}^{\int_{0}^{\breve{\tau}} F_{0}\left(X_{s}\right) \mathrm{d} s}\left(\tilde{\Psi}\left(X_{\breve{\tau}}\right)-\Psi\left(X_{\breve{\tau}}\right)\right)\right] \\
& \geq \min _{\partial \mathcal{B}}(\tilde{\Psi}-\Psi) \quad \forall x \in \mathcal{B}^{c} .
\end{aligned}
$$

It follows that $\tilde{\Psi}-\Psi$ attains a global minimum on $\overline{\mathcal{B}}$. Which means that we can scale $\Psi$ until it touches $\tilde{\Psi}$ from below in at least one point in $\overline{\mathcal{B}}$. Since

$$
\mathcal{L}(\tilde{\Psi}-\Psi)-(f-\Lambda(f))^{-}(\tilde{\Psi}-\Psi)=-(f-\Lambda(f))^{+}(\tilde{\Psi}-\Psi) \leq 0 \quad \text { on } \mathbb{R}^{d},
$$

it follows by the strong maximum principle that $\tilde{\Psi}=\Psi$ on $\mathbb{R}^{d}$. This completes the proof.

We next prove the converse statement.

Lemma 2.9. Let $\Gamma$ be as in (2.15). If process $X^{*}$ in (1.17) is recurrent, then $\Gamma(x)=\infty$ for all $x \in \mathbb{R}^{d}$.

Proof. We argue by contradiction. Suppose, without loss of generality, that $\Gamma(0)<\infty$. Following the steps in the proof of Theorem 2.8 we obtain a positive function $\Phi \in \mathcal{W}_{\mathrm{loc}}^{2, d}\left(\mathbb{R}^{d}\right)$ which satisfies

$$
\mathcal{L} \Phi(x)+F_{0}(x) \Phi(x)=-\Gamma^{-1}(0) \quad \text { a.e. } x \in \mathbb{R}^{d},
$$

and $\Phi(0)=1$.

By (2.22) and (2.23) we have

$$
\Phi(x) \leq \mathbb{E}_{x}\left[\mathrm{e}^{\mathrm{e}_{0}^{\breve{\tau}} F_{0}\left(X_{s}\right) \mathrm{d} s} \Phi\left(X_{\breve{\mathcal{\tau}}}\right)\right]+\Gamma^{-1}(0)\left(\theta \inf _{\partial \mathcal{B}} \Psi\right)^{-1} \Psi(x) \quad \forall x \in \mathcal{B}^{c} .
$$

Combining this with (2.24) and Lemma 2.6 we deduce that $\frac{\Phi}{\Psi}$ is bounded above in $\mathbb{R}^{d}$. We also have

$$
\mathcal{L}^{*} \frac{\Phi}{\Psi}=-\Gamma^{-1}(0) \Psi^{-1} \quad \text { on } \mathbb{R}^{d} .
$$

Therefore $\frac{\Phi}{\Psi}\left(X_{t}^{*}\right)$ is an $\mathfrak{F}_{t}^{X^{*}}$-supermartingale, and thus converges a.s. Since the $X^{*}$ process is recurrent the limit must be a constant, which implies that $\Psi=\Phi$. This of course is only possible if $\Gamma^{-1}(0)=0$, which is a contradiction. This completes the proof.

Lemma 2.10. Under (H1), the following hold: 
(i) For any $r>0$ the Dirichlet eigensolutions $\left(\widehat{\Psi}_{n}, \hat{\lambda}_{n}\right)$ in (2.2) have the stochastic representation

$$
\widehat{\Psi}_{n}(x)=\mathbb{E}_{x}\left[\mathrm{e}^{\int_{0}^{\breve{\tau}_{r}}\left[f\left(X_{t}\right)-\hat{\lambda}_{n}\right] \mathrm{d} t} \widehat{\Psi}_{n}\left(X_{\breve{\tau}_{r}}\right) \mathbb{1}\left\{\breve{\tau}_{r}<\tau_{n}\right\}\right] \quad \forall x \in B_{n} \backslash \bar{B}_{r},
$$

for all large enough $n \in \mathbb{N}$.

(ii) Suppose that for some ball $\mathcal{B} \subset \mathbb{R}^{d}$ and a constant $\delta>0$, we have

$$
\mathbb{E}_{x}\left[\mathrm{e}^{\left.\int_{0}^{\tau\left(\mathcal{B}^{c}\right)}\left[f\left(X_{s}\right)-\Lambda(f)+\delta\right] \mathrm{d} s\right]}<\infty \quad \forall x \in \overline{\mathcal{B}}^{c} .\right.
$$

Then the solution $\Psi$ of (2.3) satisfies

$$
\Psi(x)=\mathbb{E}_{x}\left[\mathrm{e}^{\int_{0}^{\breve{\tau}_{r}}\left[f\left(X_{s}\right)-\Lambda(f)\right] \mathrm{d} s} \Psi\left(X_{\breve{\tau}_{r}}\right)\right] \quad \forall r>0, \quad \forall x \in B_{r}^{c} .
$$

Proof. Let $\tilde{f}:=f-\delta \mathbb{1}_{B_{r}}$, and $\hat{\lambda}_{n}(\tilde{f}), \hat{\lambda}_{n}(f)$ denote the Dirichlet eigenvalues on $B_{n}$ corresponding to $\tilde{f}, f$, respectively. By the strict monotonicity of the Dirichlet eigenvalue in Lemma 2.2(a), we have $\hat{\lambda}_{n}(\tilde{f})<\hat{\lambda}(f)$ for all $n \in \mathbb{N}$. It then follows by the continuity of the Dirichlet eigenvalue with respect to the radius of the ball, again in Lemma 2.2(a), that there exists a ball $B_{r_{n}} \ni B_{n}$ such that $\hat{\lambda}_{r_{n}}(\tilde{f})<\hat{\lambda}_{n}(f)$. Define $\delta_{n}:=\hat{\lambda}_{n}(f)-\hat{\lambda}_{r_{n}}(\tilde{f})>0$. Let $\left(\widehat{\Psi}_{r_{n}}^{\tilde{f}}, \hat{\lambda}_{r_{n}}(\tilde{f})\right)$, and $\left(\widehat{\Psi}_{n}, \hat{\lambda}_{n}(f)\right)$, denote the Dirichlet eigensolutions on $B_{r_{n}}$ for $\tilde{f}$, and on $B_{n}$ for $f$, respectively. In the interest of simplifying the equations, we drop the explicit dependence on $n$, and adopt the following simplified notation:

$$
\begin{gathered}
\widehat{\Psi}=\widehat{\Psi}_{n}, \quad \tilde{\Psi}=\widehat{\Psi}_{r_{n}}^{\tilde{f}}, \quad \hat{\lambda}=\hat{\lambda}_{n}(f), \quad \tilde{\lambda}=\hat{\lambda}_{r_{n}}(\tilde{f}), \quad \delta:=\hat{\lambda}-\tilde{\lambda}>0, \\
D=B_{n}, \quad \tilde{D}=B_{r_{n}}, \quad \tau=\tau\left(B_{n}\right) .
\end{gathered}
$$

Then

$$
\begin{array}{lll}
\mathcal{L} \tilde{\Psi}(x)+\tilde{f}(x) \tilde{\Psi}(x)=(\hat{\lambda}-\delta) \tilde{\Psi}(x) \quad \text { a.e. } x \in \tilde{D}, & \text { and } \quad \tilde{\Psi}(0)=1,\left.\tilde{\Psi}\right|_{\partial \tilde{D}}=0 \\
\mathcal{L} \widehat{\Psi}(x)+f(x) \widehat{\Psi}(x)=\hat{\lambda} \widehat{\Psi}(x) \quad \text { a.e. } x \in D, \quad \text { and } \quad \widehat{\Psi}(0)=1,\left.\widehat{\Psi}\right|_{\partial D}=0 .
\end{array}
$$

Applying Dynkin's formula to the first equation in (2.26), for the stopping time $T \wedge \tau \wedge \breve{\tau}_{r}$, we obtain

$$
\begin{aligned}
\tilde{\Psi}(x) & =\mathbb{E}_{x}\left[\mathrm{e}^{\int_{0}^{T \wedge \tau \wedge \breve{\tau}_{r}}\left[\tilde{f}\left(X_{t}\right)-\hat{\lambda}+\delta\right] \mathrm{d} t} \tilde{\Psi}\left(X_{T \wedge \tau \wedge \breve{\tau}_{r}}\right)\right] \\
& \geq \mathbb{E}_{x}\left[\mathrm{e}^{\int_{0}^{T}\left[\tilde{f}\left(X_{t}\right)-\hat{\lambda}+\delta\right] \mathrm{d} t} \tilde{\Psi}\left(X_{T}\right) \mathbb{1}\left\{T<\tau \wedge \breve{\tau}_{r}\right\}\right]
\end{aligned}
$$

Therefore we have

$$
\begin{aligned}
\mathbb{E}_{x}\left[\mathrm{e}^{\int_{0}^{T}\left[\tilde{f}\left(X_{t}\right)-\hat{\lambda}\right] \mathrm{d} t} \widehat{\Psi}\left(X_{T}\right) \mathbb{1}\left\{T<\tau \wedge \breve{\tau}_{r}\right\}\right] & \\
& \leq \mathrm{e}^{-\delta T}\left(\sup _{D} \widehat{\Psi}\right)\left(\inf _{D} \tilde{\Psi}\right)^{-1} \tilde{\Psi}(x) \quad \forall T>0, \quad \forall x \in D .
\end{aligned}
$$

Thus, applying Dynkin's formula to the second equation in (2.26), we obtain

$$
\begin{aligned}
\widehat{\Psi}(x)= & \lim _{T \rightarrow \infty} \mathbb{E}_{x}\left[\mathrm{e}^{\int_{0}^{T}\left[f\left(X_{t}\right)-\hat{\lambda}\right] \mathrm{d} t} \widehat{\Psi}\left(X_{T}\right) \mathbb{1}\left\{T<\tau \wedge \breve{\tau}_{r}\right\}\right] \\
& \quad+\lim _{T \rightarrow \infty} \mathbb{E}_{x}\left[\mathrm{e}^{\int_{0}^{\tau \wedge \breve{\tau}_{r}}\left[f\left(X_{t}\right)-\hat{\lambda}\right] \mathrm{d} t} \widehat{\Psi}\left(X_{\tau \wedge \breve{\tau}_{r}}\right) \mathbb{1}\left\{T \geq \tau \wedge \breve{\tau}_{r}\right\}\right] \\
= & \mathbb{E}_{x}\left[\mathrm{e}^{\int_{0}^{\tau \wedge \breve{\tau}_{r}}\left[f\left(X_{t}\right)-\hat{\lambda}\right] \mathrm{d} t} \widehat{\Psi}\left(X_{\tau \wedge \breve{\tau}_{r}}\right)\right] \\
= & \mathbb{E}_{x}\left[\mathrm{e}^{\int_{0}^{\breve{\tau}_{r}}\left[f\left(X_{t}\right)-\hat{\lambda}\right] \mathrm{d} t} \widehat{\Psi}\left(X_{\breve{\tau}_{r}}\right) \mathbb{1}\left\{\breve{\tau}_{r}<\tau\right\}\right],
\end{aligned}
$$


where for the first limit we use (2.27), for the second limit we use monotone convergence, while for the last equality we use the property that $\widehat{\Psi}=0$ on $\partial D$. This proves part (i).

Now we prove (2.25). Applying Fatou's lemma to (2.28) we obtain, with $\breve{\tau}=\tau\left(\mathcal{B}^{c}\right)$ that

$$
\Psi(x) \geq \mathbb{E}_{x}\left[\mathrm{e}^{\int_{0}^{\breve{\tau}}\left[f\left(X_{t}\right)-\Lambda(f)\right] \mathrm{d} t} \Psi\left(X_{\breve{\tau}}\right)\right] .
$$

We write (2.28) as

$$
\begin{aligned}
\widehat{\Psi}_{n}(x)= & \mathbb{E}_{x}\left[\mathrm{e}^{\int_{0}^{\breve{\tau}}\left[f\left(X_{t}\right)-\hat{\lambda}_{n}\right] \mathrm{d} t} \Psi\left(X_{\breve{\tau})} \mathbb{1}\left\{\breve{\tau}<\tau_{n}\right\}\right]\right. \\
& +\left(\sup _{B_{r}}\left|\Psi-\widehat{\Psi}_{n}\right|\right) \mathbb{E}_{x}\left[\mathrm{e}^{\int_{0}^{\breve{\tau}}\left[f\left(X_{t}\right)-\hat{\lambda}_{n}\right] \mathrm{d} t} \mathbb{1}\left\{\breve{\tau}<\tau_{n}\right\}\right] \\
\leq & \mathbb{E}_{x}\left[\mathrm{e}^{\int_{0}^{\breve{\tau}}\left[f\left(X_{t}\right)-\hat{\lambda}_{n}\right] \mathrm{d} t} \Psi\left(X_{\breve{\tau})}\right]+\left(\sup _{B_{r}}\left|\Psi-\widehat{\Psi}_{n}\right|\right) \mathbb{E}_{x}\left[\mathrm{e}^{\int_{0}^{\breve{\tau}}\left[f\left(X_{t}\right)-\hat{\lambda}_{n}\right] \mathrm{d} t} \mathbb{1}\left\{\breve{\tau}<\tau_{n}\right\}\right] .\right.
\end{aligned}
$$

Note that since $\hat{\lambda}_{n} \nearrow \Lambda(f)$, the first term on the right hand side of (2.30) is finite for all large enough $n$ by Lemma 2.3(iii). Let

$$
\kappa_{n}:=\left(\inf _{B_{r}} \widehat{\Psi}_{n}\right)^{-1}\left(\sup _{B_{r}}\left|\Psi-\widehat{\Psi}_{n}\right|\right)
$$

The second term on the right hand side of (2.30) has the bound

$$
\begin{aligned}
\left(\sup _{B_{r}}\left|\Psi-\widehat{\Psi}_{n}\right|\right) \mathbb{E}_{x}\left[\mathrm{e}^{\int_{0}^{\breve{\tau}}\left[f\left(X_{t}\right)-\hat{\lambda}_{n}\right] \mathrm{d} t} \mathbb{1}\left\{\breve{\tau}<\tau_{n}\right\}\right] & \leq \kappa_{n} \mathbb{E}_{x}\left[\mathrm{e}^{\int_{0}^{\breve{\tau}}\left[f\left(X_{t}\right)-\hat{\lambda}_{n}\right] \mathrm{d} t} \widehat{\Psi}_{n}\left(X_{\breve{\tau}}\right) \mathbb{1}\left\{\breve{\tau}<\tau_{n}\right\}\right] \\
& =\kappa_{n} \widehat{\Psi}_{n}(x) .
\end{aligned}
$$

By the convergence of $\widehat{\Psi}_{n} \rightarrow \Psi$ as $n \rightarrow \infty$, uniformly on compact sets, and since $\widehat{\Psi}_{n}$ is bounded away from 0 in $B_{r}$, uniformly in $n \in \mathbb{N}$ by the Harnack inequality, we have $\kappa_{n} \rightarrow 0$ as $n \rightarrow \infty$. Therefore, the second term on the right hand side of (2.30) vanishes as $n \rightarrow \infty$. Also, since $\hat{\lambda}_{n}$ is nondecreasing in $n$, and $\hat{\lambda}_{n} \nearrow \Lambda(f)$, we have

$$
\mathbb{E}_{x}\left[\mathrm{e}^{\int_{0}^{\breve{\tau}}\left[f\left(X_{t}\right)-\hat{\lambda}_{n}\right] \mathrm{d} t} \Psi\left(X_{\breve{\tau})}\right] \underset{n \rightarrow \infty}{\longrightarrow} \mathbb{E}_{x}\left[\mathrm{e}^{\int_{0}^{\breve{\tau}}\left[f\left(X_{t}\right)-\Lambda(f)\right] \mathrm{d} t} \Psi\left(X_{\breve{\tau}}\right)\right]\right.
$$

by monotone convergence. Thus taking limits in (2.30) as $n \rightarrow \infty$, and using (2.29) and (2.31) we obtain (2.16). This implies that (2.25) holds for any $r>0$ by Lemma 2.6. This completes the proof.

Lemma 2.11. If the process $X^{*}$ in (1.17) is not recurrent, then for any bounded measurable set $A \in \mathbb{R}^{d}$ of positive Lebesgue measure there exists $\epsilon>0$ such that $\Lambda\left(f+\epsilon \mathbb{1}_{A}\right)=\Lambda(f)$. On the other hand, if $X^{*}$ is recurrent then $\Lambda(f)<\Lambda\left(f+\epsilon \mathbb{1}_{A}\right)$ for all measurable set $A \in \mathbb{R}^{d}$ of positive Lebesgue measure and $\epsilon>0$.

Proof. Let $A \in \mathbb{R}^{d}$ be a bounded measurable set, and $\mathcal{B}$ and open ball containing $A$. We set $\breve{\tau}=\tau\left(\mathcal{B}^{c}\right)$. Recall the eigenvalues $\left\{\hat{\lambda}_{n}\right\}$ in Lemma 2.2 Since $\hat{\lambda}_{n}<\Lambda(f)$, the principal eigenvalue of the operator $-\mathcal{L}-(f-\Lambda(f))$ on every $B_{n}$ is positive. Thus by Proposition 6.2 and Theorem 6.1 in [8] , for any $n \in \mathbb{N}$, and $\alpha_{n} \geq 0$, the Dirichlet problem

$$
\mathcal{L} \varphi_{n}+(f-\Lambda(f)) \varphi_{n}=-\alpha_{n} \mathbb{1}_{A} \quad \text { in } B_{n},
$$

with $\varphi_{n}=0$ on $\partial B_{n}$, has a unique solution $\varphi_{n} \in \mathcal{W}_{\text {loc }}^{2, p}\left(B_{n}\right) \cap \mathcal{C}\left(\bar{B}_{n}\right)$, for any $p \geq 1$. In addition, provided $\alpha_{n} \geq 0$, then by the refined maximum principle in [8, Theorem 1.1] $\varphi_{n}$ is nonnegative. Let $\tilde{\alpha}_{n}>0$ be such that the Dirichlet problem $\mathcal{L} \tilde{\varphi}_{n}+(f-\Lambda(f)) \tilde{\varphi}_{n}=-\tilde{\alpha}_{n} \mathbb{1}_{A}$ in $B_{n}$, with $\tilde{\varphi}_{n}=0$ on $\partial B_{n}$, satisfies $\tilde{\varphi}_{n}(0)=1$. We set $\alpha_{n}=\min \left(1, \tilde{\alpha}_{n}\right)$ in (2.32). Thus, the assumptions are met for the Harnack inequality for a class of superharmonic functions in [1], and we have

$$
\varphi_{n}(x) \leq C_{H} \quad \forall x \in B_{n}, \quad \forall n \in \mathbb{N},
$$


for some constant $C_{H}$ depending on $n$. Thus the collection $\left\{\varphi_{n}, n \in \mathbb{N}\right\}$ is relatively weakly compact in $\mathcal{W}^{2, p}\left(B_{R}\right)$ for any $p \geq 1$ and $R>0$, and taking limits along some sequence $n \rightarrow \infty$, we obtain a positive function $\Phi \in \mathcal{W}_{\text {loc }}^{2, d}\left(\mathbb{R}^{d}\right)$, which solves

$$
\mathcal{L} \Phi+(f-\Lambda(f)) \Phi=-\alpha \mathbb{1}_{A}
$$

on $\mathbb{R}^{d}$, for some nonnegative constant $\alpha$. If $\alpha>0$ then $\Phi$ is positive on $\mathbb{R}^{d}$ by the strong maximum principle. On the other hand, if $\alpha_{n} \searrow 0$, then the definition of $\alpha, \varphi_{n}(0)=1$ along this sequence, except for a finite number of terms, since $\varphi_{n}(0)=1$ whenever $\alpha_{n}<1$. Thus, in either case, the solution $\Phi$ is positive.

By Itô's formula applied to (2.32), with $\breve{\tau}=\tau\left(\mathcal{B}^{c}\right)$, we obtain

$$
\begin{aligned}
\varphi_{n}(x)=\mathbb{E}_{x}\left[\mathrm{e}^{\int_{0}^{\breve{\tau}}\left[f\left(X_{s}\right)-\Lambda(f)\right] \mathrm{d} s} \varphi_{n}\left(X_{\breve{\tau})} \mathbb{1}_{\left\{\breve{\tau}<T \wedge \tau_{n}\right\}}\right]\right. \\
+\mathbb{E}_{x}\left[\mathrm{e}^{\int_{0}^{T}\left[f\left(X_{s}\right)-\Lambda(f)\right] \mathrm{d} s} \varphi_{n}\left(X_{T}\right) \mathbb{1}_{\left\{T<\breve{\tau} \wedge \tau_{n}\right\}}\right] \quad \forall x \in B_{n} \backslash \mathcal{B}, \forall T>0 .
\end{aligned}
$$

With $\left(\widehat{\Psi}_{n}, \hat{\lambda}_{n}\right)$ denoting the Dirichlet eigensolutions in (2.2), and Itô's formula, we obtain

$$
\mathbb{E}_{x}\left[\mathrm{e}^{\int_{0}^{T}\left[f\left(X_{s}\right)-\hat{\lambda}_{n+1}\right] \mathrm{d} s} \mathbb{1}_{\left\{T<\breve{\tau} \wedge \tau_{n}\right\}}\right] \leq\left(\inf _{B_{n} \backslash \mathcal{B}} \widehat{\Psi}_{n+1}\right)^{-1} \widehat{\Psi}_{n+1}(x) \quad \forall T>0, \forall x \in B_{n} \backslash \mathcal{B} .
$$

Thus, we have

$$
\begin{aligned}
& \mathbb{E}_{x}\left[\mathrm{e}^{\int_{0}^{T}\left[f\left(X_{s}\right)-\Lambda(f)\right] \mathrm{d} s} \varphi_{n}\left(X_{T}\right) \mathbb{1}_{\left\{T<\breve{\tau} \wedge \tau_{n}\right\}}\right] \\
& \quad \leq\left\|\varphi_{n}\right\|_{\infty} \mathrm{e}^{\left(\hat{\lambda}_{n+1}-\Lambda(f)\right) T} \mathbb{E}_{x}\left[\mathrm{e}^{\int_{0}^{T}\left[f\left(X_{s}\right)-\hat{\lambda}_{n+1}\right] \mathrm{d} s} \mathbb{1}_{\left\{T<\breve{\tau} \wedge \tau_{n}\right\}}\right] \\
& \quad \leq\left\|\varphi_{n}\right\|_{\infty} \mathrm{e}^{\left(\hat{\lambda}_{n+1}-\Lambda(f)\right) T}\left(\inf _{B_{n} \backslash \mathcal{B}} \widehat{\Psi}_{n+1}\right)^{-1} \widehat{\Psi}_{n+1}(x) \quad \forall T>0,
\end{aligned}
$$

and all $x \in B_{n}$, and it follows that the second term on the right hand side of (2.34) vanishes as $T \rightarrow \infty$. Therefore, taking limits as $T \rightarrow \infty$ in (2.34), we have

$$
\varphi_{n}(x)=\mathbb{E}_{x}\left[\mathrm{e}^{\int_{0}^{\breve{\tau}}\left[f\left(X_{s}\right)-\Lambda(f)\right] \mathrm{d} s} \varphi_{n}\left(X_{\breve{\tau}}\right) \mathbb{1}_{\left\{\breve{\tau}<\tau_{n}\right\}}\right] \quad \forall x \in B_{n} \backslash \mathcal{B},
$$

and taking again limits as $n \rightarrow \infty$ in (2.35), we obtain

$$
\Phi(x)=\mathbb{E}_{x}\left[\mathrm{e}^{\int_{0}^{\breve{\tau}}\left[f\left(X_{s}\right)-\Lambda(f)\right] \mathrm{d} s} \Phi\left(X_{\breve{\tau}}\right) \mathbb{1}_{\{\breve{\tau}<\infty\}}\right] \quad \forall x \in \mathcal{B}^{c} .
$$

Suppose that $X^{*}$ in (1.17) is not recurrent. Then we claim that $\alpha>0$. Indeed, if $\alpha=0$, then $\Phi$ is a ground state corresponding to $f$, and Lemma 2.6 together with (2.36) imply that $X^{*}$ is recurrent, which contradicts the hypothesis. Therefore, writing (2.33) as

$$
\mathcal{L} \Phi+\left(f+\alpha \Phi^{-1} \mathbb{1}_{A}-\Lambda(f)\right) \Phi=0
$$

and letting $\epsilon:=\inf _{\mathcal{B}} \alpha \Phi^{-1}$, it follows that $\Lambda\left(f+\epsilon \mathbb{1}_{A}\right)=\Lambda(f)$.

Next assume $X^{*}$ in (1.17) is recurrent, and suppose that $\Lambda\left(f+\epsilon \mathbb{1}_{A}\right)=\Lambda(f)$ for some $\epsilon>0$. We repeat the construction in the first part of the proof, starting from the Dirichlet problem

$$
\mathcal{L} \varphi_{n}+\left(f+\epsilon \mathbb{1}_{A}-\Lambda(f)\right) \varphi_{n}=-\alpha_{n} \mathbb{1}_{A} \quad \text { in } B_{n},
$$

with $\varphi_{n}=0$ on $\partial B_{n}$, to obtain positive function $\Phi \in \mathcal{W}_{\text {loc }}^{2, d}\left(\mathbb{R}^{d}\right)$, which solves

$$
\mathcal{L} \Phi+\left(f+\left(\epsilon+\alpha \Phi^{-1}\right) \mathbb{1}_{A}-\Lambda(f)\right) \Phi=0
$$


for some $\alpha \geq 0$, and satisfies (2.36). Since $X^{*}$ is recurrent, the ground state $\Psi$ corresponding to $f$ satisfies (2.36) by Lemma 2.6. Thus

$$
\Phi(x)-\Psi(x) \geq \min _{\mathcal{B}}(\Phi-\Psi) \quad \forall x \in \mathcal{B}^{c},
$$

and by scaling $\Phi$ so that it touches $\Psi$ at one point from above, and using the strong maximum principle we deduce that $\Psi=\Phi$. This of course is impossible unless $\epsilon=0$ and $\alpha=0$, hence we reach a contradiction. The proof is complete.

\subsection{Results concerning Theorems $1 . 6 \longdiv { 1 . 8 }$}

We start with the following theorem.

Theorem 2.12. Under (H1) the following are equivalent.

(a) For some ball $\mathcal{B} \subset \mathbb{R}^{d}$ and a constant $\delta>0$, we have

$$
\mathbb{E}_{x}\left[\mathrm{e}^{\left.\int_{0}^{\tau\left(\mathcal{B}^{c}\right)}\left[f\left(X_{s}\right)-\Lambda(f)+\delta\right] \mathrm{d} s\right]}<\infty \quad \forall x \in \overline{\mathcal{B}}^{c} .\right.
$$

(b) If $h \in \mathcal{C}_{c}\left(\mathbb{R}^{d}\right)$ is non-negative function, $h \not \equiv 0$, then $\Lambda(f)>\Lambda(f-h)$.

(c) For every $r>0$, there exists $\delta_{r}>0$, such that

$$
\mathbb{E}_{x}\left[\mathrm{e}^{\int_{0}^{\breve{r} r}\left[f\left(X_{s}\right)-\Lambda(f)+\delta_{r}\right] \mathrm{d} s}\right]<\infty \quad \forall x \in \bar{B}_{r}^{c} .
$$

Proof. The proof $(\mathrm{a}) \Rightarrow(\mathrm{b})$ is by contradiction. Without loss of generality suppose that $\mathcal{B}$ contains the support of $h$, and define $\tilde{f}=f-h$. It is clear that $\Lambda(f) \geq \Lambda(\tilde{f})$, so suppose that $\Lambda(f)=\Lambda(\tilde{f})$. Let $\tilde{\Psi}$ be a positive solution to

$$
\mathcal{L} \tilde{\Psi}(x)+\tilde{f}(x) \tilde{\Psi}(x)=\Lambda(f) \tilde{\Psi}(x) \quad \text { a.e. } x \in \mathbb{R}^{d} .
$$

If $\Psi$ is a positive solution to (2.3), then we have

$$
\mathcal{L} \Psi(x)+(\tilde{f}(x)-\Lambda(f)) \Psi(x)=-h(x) \Psi(x) \quad \text { a.e. } x \in \mathbb{R}^{d} .
$$

By (a) and Lemma 2.10, we have

$$
\tilde{\Psi}(x)=\mathbb{E}_{x}\left[\mathrm{e}^{\int_{0}^{\tau\left(\mathcal{B}^{c}\right)}\left[f\left(X_{s}\right)-\Lambda(f)\right] \mathrm{d} s} \tilde{\Psi}\left(X_{\tau\left(\mathcal{B}^{c}\right)}\right)\right] \quad \forall x \in \overline{\mathcal{B}}^{c} .
$$

Therefore, as in the proof of uniqueness in Theorem 2.8 we have

$$
\Psi(x)-\tilde{\Psi}(x) \geq \min _{\mathcal{B}}(\Psi-\tilde{\Psi}) \quad \forall x \in \mathcal{B}^{c},
$$

which means that we can scale $\Psi$ until it touches $\tilde{\Psi}$ from above in at least one point in $\mathcal{B}$. Since

$$
\mathcal{L}(\Psi-\tilde{\Psi})-(f-\Lambda(f))^{-}(\Psi-\tilde{\Psi})=-(f-\Lambda(f))^{+}(\Psi-\tilde{\Psi})-h \tilde{\Psi} \leq 0 \quad \text { on } \quad \mathbb{R}^{d}
$$

it follows by the strong maximum principle that $\Psi=\tilde{\Psi}$ on $\mathbb{R}^{d}$. However, $\Psi$ solves $\mathcal{L} \Psi=(\Lambda(f)-f) \Psi$ on $\mathbb{R}^{d}$, and this implies that $h \tilde{\Psi}=0$ a.e. This, of course, is not possible since $\tilde{\Psi}$ is positive. Thus we reached a contradiction, and the proof of part (a) is complete.

Let $r>0$, and $\tilde{\Psi}$ be a positive solution of (2.3) corresponding to $\tilde{f}=f-\mathbb{1}_{B_{r}}$. By part (b), we have $\delta_{r}:=\Lambda(f)-\Lambda(\tilde{f})>0$. Applying Itô's formula and Fatou's lemma to $\tilde{\Psi}$, we obtain (2.38). This shows (b) $\Rightarrow(\mathrm{c})$, and since it is clear that $(\mathrm{c}) \Rightarrow$ (a) the proof is complete. 
Recall that $\mathbb{E}_{x}^{*}$ denotes the expectation operator associated with $X^{*}$ in (1.17), while $\mathbb{E}_{x}$ and $\mu$ denote the expectation operator and invariant probability measure associated with the solution of (1.12), respectively.

Theorem 2.13. Assume (H1), and suppose that the sde in (1.17) has a unique strong solution $X^{*}$ which exists for all $t>0$. The following hold for any function $g \in \mathcal{C}_{b}\left(\mathbb{R}^{d}\right)$, and all $(t, x) \in \mathbb{R}_{+} \times \mathbb{R}^{d}$ :

$$
\begin{aligned}
\Psi(x) \mathbb{E}_{x}^{*}\left[g\left(X_{t}^{*}\right) \Psi^{-1}\left(X_{t}^{*}\right)\right] & =\mathbb{E}_{x}\left[\mathrm{e}^{\int_{0}^{t}\left[f\left(X_{s}\right)-\Lambda(f)\right] \mathrm{d} s} g\left(X_{t}\right)\right], \\
\Psi(x) \mathbb{E}_{x}^{*}\left[g\left(X_{t}^{*}\right)\right] & =\mathbb{E}_{x}\left[\mathrm{e}^{\int_{0}^{t}\left[f\left(X_{s}\right)-\Lambda(f)\right] \mathrm{d} s} \Psi\left(X_{t}\right) g\left(X_{t}\right)\right],
\end{aligned}
$$

where $\Psi$ is a positive solution of (2.3). In particular, we have

$$
\Psi(x)=\mathbb{E}_{x}\left[\mathrm{e}^{\int_{0}^{t}\left[f\left(X_{s}\right)-\Lambda(f)\right] \mathrm{d} s} \Psi\left(X_{t}\right)\right] \quad \forall(t, x) \in \mathbb{R}_{+} \times \mathbb{R}^{d} .
$$

Suppose that $X^{*}$ is positive recurrent and let $\mu^{*}$ denote its invariant probability measure. Then

$$
\mathbb{E}_{x}\left[\mathrm{e}^{\int_{0}^{t}\left[f\left(X_{s}\right)-\Lambda(f)\right] \mathrm{d} s} \Psi\left(X_{t}\right) g\left(X_{t}\right)\right] \underset{t \rightarrow \infty}{\longrightarrow} \Psi(x) \int_{\mathbb{R}^{d}} g(y) \mu^{*}(\mathrm{~d} y)
$$

for all $g \in \mathcal{C}_{b}\left(\mathbb{R}^{d}\right)$. In particular, $\Psi_{*}$ defined by

$$
\Psi_{*}(x):=\lim _{T \rightarrow \infty} \mathbb{E}_{x}\left[\mathrm{e}^{\int_{0}^{T}\left[f\left(X_{t}\right)-\Lambda(f)\right] \mathrm{d} t}\right],
$$

is a positive solution of the MPE and satisfies $\mu^{*}\left(\Psi_{*}^{-1}\right)=1$.

ProOF. Recall (2.10), which we repeat here as

$$
\begin{aligned}
\mathbb{E}_{x}\left[\mathrm{e}^{\int_{0}^{t \wedge \tau_{n}}\left[f\left(X_{s}\right)-\Lambda(f)\right] \mathrm{d} s} g\left(X_{t \wedge \tau_{n}}\right)\right] & =\Psi(x) \mathbb{E}_{x}\left[g\left(X_{t \wedge \tau_{n}}\right) \Psi^{-1}\left(X_{t \wedge \tau_{n}}\right) \mathcal{M}_{t \wedge \tau_{n}}\right] \\
& =\Psi(x) \mathbb{E}_{x}^{*}\left[g\left(X_{t \wedge \tau_{n}}^{*}\right) \Psi^{-1}\left(X_{t \wedge \tau_{n}}^{*}\right)\right] .
\end{aligned}
$$

Let $g \in \mathcal{C}_{c}\left(\mathbb{R}^{d}\right)$, and note that since $\Psi$ is inf-compact, the term inside the expectation in the right hand side of (2.43) is bounded uniformly in $n$. Thus letting $n \rightarrow \infty$ in (2.43), we obtain

$$
\begin{aligned}
\mathbb{E}_{x}\left[\mathrm{e}^{\int_{0}^{t}\left[f\left(X_{s}\right)-\Lambda(f)\right] \mathrm{d} s} g\left(X_{t}\right)\right] & =\Psi(x) \mathbb{E}_{x}\left[g\left(X_{t}\right) \Psi^{-1}\left(X_{t}\right) \mathcal{M}_{t}\right] \\
& =\Psi(x) \mathbb{E}_{x}^{*}\left[g\left(X_{t}^{*}\right) \Psi^{-1}\left(X_{t}^{*}\right)\right] \quad \forall t>0 .
\end{aligned}
$$

This proves (2.39) for $g \in \mathcal{C}_{c}\left(\mathbb{R}^{d}\right)$, and also for $g \in \mathcal{C}_{b}\left(\mathbb{R}^{d}\right)$ by monotone convergence over an increasing sequence $g_{n} \in \mathcal{C}_{c}\left(\mathbb{R}^{d}\right)$.

Applying again monotone convergence to (2.44) we obtain (2.40). By Lemma 2.5. $\left(\mathcal{M}_{t}, \mathfrak{F}_{t}^{X}\right)$ is a martingale, and thus (2.41) follows by Lemma 2.4

Since $X^{*}$ is ergodic, we obtain from (2.40) that

$$
\begin{aligned}
\lim _{T \rightarrow \infty} \frac{1}{T} \int_{0}^{T} \mathbb{E}_{x}\left[\mathrm{e}^{\int_{0}^{T}\left(f\left(X_{s}\right)-\Lambda(f)\right) \mathrm{d} s} \Psi\left(X_{T}\right) g\left(X_{T}\right)\right] & =\Psi(x) \lim _{T \rightarrow \infty} \frac{1}{T} \int_{0}^{T} \mathbb{E}_{x}^{*}\left[g\left(X_{t}^{*}\right)\right] \\
& =\Psi(x) \int_{\mathbb{R}^{d}} g(y) \mu^{*}(\mathrm{~d} y)
\end{aligned}
$$

by Birkhoff's ergodic theorem. Then (2.42) follows by an application of [33, Theorem 4.12]. This completes the proof. 
Corollary 2.14. Assume (H1). Then there exist an open ball $\mathcal{B} \subset \mathbb{R}^{d}$ and a constant $\delta>0$ such that (2.37) holds, if and only if the ground state diffusion $X^{*}$ is geometrically ergodic.

Proof. We first show necessity. By Theorem 2.12, for any $r>0$, we have $\delta:=\Lambda(f)-\Lambda\left(f-\gamma \mathbb{1}_{B_{r}}\right)>0$. Let $\tilde{\Psi}$ be the positive solution of the MPE

$$
\mathcal{L} \tilde{\Psi}=\left(\Lambda\left(f-\gamma \mathbb{1}_{B_{r}}\right)-f+\gamma \mathbb{1}_{B_{r}}\right) \widetilde{\Psi} .
$$

With $\tilde{\mathcal{V}}=\tilde{\Psi} \Psi^{-1}$, we have

$$
\begin{aligned}
\mathcal{L}^{*} \tilde{\mathcal{V}} & =\left(\Lambda\left(f-\gamma \mathbb{1}_{B_{r}}\right)-f+\gamma \mathbb{1}_{B_{r}}-\Lambda(f)+f\right) \tilde{\mathcal{V}} \\
& =\left(\gamma \mathbb{1}_{B_{r}}-\delta\right) \tilde{\mathcal{V}} .
\end{aligned}
$$

It follows by Lemma 2.10(ii) that $\inf _{\mathbb{R}^{d}} \tilde{\mathcal{V}}>0$. Thus, the Foster-Lyapunov equation in (2.45) implies that (1.17) is geometrically ergodic.

Next suppose that $X^{*}$ is geometrically ergodic. This implies that for some open ball $\mathcal{B} \subset \mathbb{R}^{d}$ and a constant $\delta>0$ we have $\mathbb{E}_{x}^{*}\left[\mathrm{e}^{\delta \tau}\right]<\infty$ for all $x \in \mathcal{B}^{c}$, where $\breve{\tau}=\tau\left(\mathcal{B}^{c}\right)$. We may select $\mathcal{B}$ so that $\mathcal{B} \supset \mathcal{K}$ in Definition 1.1. Similarly to (2.43) we obtain

$$
\mathbb{E}_{x}\left[\mathrm{e}^{\int_{0}^{\breve{\tau} \wedge \tau_{n}}\left[f\left(X_{s}\right)-\Lambda(f)+\delta\right] \mathrm{d} s} \Psi\left(X_{\breve{\tau} \wedge \tau_{n}}\right)\right]=\Psi(x) \mathbb{E}_{x}^{*}\left[\mathrm{e}^{\delta\left(\breve{\tau} \wedge \tau_{n}\right)}\right] .
$$

Thus (2.37) follows by letting $n \rightarrow \infty$ in (2.46), and using the fact that $\inf _{\mathbb{R}^{d}} \Psi>0$. This completes the proof.

Lemma 2.15. Under (H1)-(H2) the ground state diffusion in (1.17) is geometrically ergodic.

Proof. By (H2) there exist a nonnegative constant $\kappa_{0}$ and an inf-compact function $g_{0}: \mathbb{R}^{d} \rightarrow \mathbb{R}_{+}$such that

$$
\frac{1}{2} a^{i j} \partial_{i j} \psi_{0}+\left\langle b, \nabla \psi_{0}\right\rangle+\frac{1}{2}\left\langle\nabla \psi_{0}, a \nabla \psi_{0}\right\rangle+f=\kappa_{0}-g_{0} .
$$

Thus, with $\Psi_{0}=\mathrm{e}^{\psi_{0}}$ we obtain

$$
\mathcal{L} \Psi_{0}=\left(\kappa_{0}-g_{0}-f\right) \Psi_{0} .
$$

The argument used in the proof of Lemma 2.1 shows that $\Psi_{0}$ is inf-compact, and therefore $\inf _{\mathbb{R}^{d}} \Psi_{0}>0$. Let $\mathcal{B}$ be a ball such that $g_{0} \geq \kappa_{0}-\Lambda(f)+\delta$ on $\mathcal{B}^{c}$, for some $\delta>0$. By Itô's formula and Fatou's lemma we obtain, with $\breve{\tau}=\tau\left(\mathcal{B}^{c}\right)$ that

$$
\Psi_{0}(x) \geq\left(\inf _{\mathbb{R}^{d}} \Psi_{0}\right) \mathbb{E}_{x}\left[\mathrm{e}^{\int_{0}^{\tau}\left[f\left(X_{s}\right)-\Lambda(f)+\delta\right] \mathrm{d} s}\right] \quad \forall x \in \overline{\mathcal{B}}^{c} .
$$

The result then follows by Corollary 2.14 .

We conclude this section with the proof of Theorem 1.8

Proof of Theorem 1.8. We claim that there exist a bounded open ball $\mathcal{B}_{\circ}$ and a positive constant $\delta_{\circ}$ such that, with $\breve{\tau}_{\circ}=\tau\left(\mathcal{B}_{\circ}^{c}\right)$ we have

$$
\mathbb{E}_{x}\left[\mathrm{e}^{\mathrm{e}_{0}^{\breve{\tau}_{0}}\left[f\left(X_{t}\right)-\Lambda(f)+\delta_{\circ}\right] \mathrm{d} t}\right]<\infty \quad \forall x \in \mathcal{B}_{\circ}^{c} .
$$

Under the hypothesis for some $\varepsilon>0, \varepsilon f$ is near monotone relative to $\Lambda((1+\varepsilon) f)-\Lambda(f)$ and so $\varepsilon(f-\Lambda(f))$ is near-monotone relative to $\Lambda((1+\varepsilon) f)-(1+\varepsilon) \Lambda(f)$. Thus, we can select some $\delta_{\circ}$ such that $f-\Lambda(f)$ is near monotone relative to $\theta(\varepsilon)$ which is defined as

$$
\theta(\varepsilon):=\frac{1}{\varepsilon}\left((1+\varepsilon) \delta_{\circ}+\Lambda((1+\varepsilon) f)-(1+\varepsilon) \Lambda(f)\right) .
$$


Let $\mathcal{B}_{\circ}$ be a bounded ball centered at the origin such that $f-\Lambda \geq \theta(\varepsilon)$ on $\mathcal{B}_{\circ}^{c}$. Let $F(t, x):=\mathrm{e}^{\theta(\varepsilon) t} \Psi$. Then $\partial / \partial t F(t, x)+\mathcal{L} F(t, x) \leq 0$ for all $x \in \mathcal{B}_{\circ}^{c}$, and a straightforward application of the Itô formula shows that

$$
\mathbb{E}_{x}\left[\mathrm{e}^{\theta(\varepsilon) \breve{\tau}_{\circ}}\right] \leq \Psi(x)\left(\inf _{\partial \mathcal{B}_{\circ}} \Psi\right)^{-1} \quad \forall x \in \mathcal{B}_{\circ}^{c}
$$

We write

$$
f-\Lambda(f)+\delta_{\circ}=\frac{\varepsilon}{1+\varepsilon} \theta(\varepsilon)+\left(f-\frac{\Lambda((1+\varepsilon) f)}{1+\varepsilon}\right),
$$

and apply Hölder's inequality to obtain

$$
\mathbb{E}_{x}\left[\mathrm{e}^{\int_{0}^{\breve{\tau}_{\circ}}\left[f\left(X_{t}\right)-\Lambda(f)+\delta_{\circ}\right] \mathrm{d} t}\right] \leq\left(\mathbb{E}_{x}\left[\mathrm{e}^{\theta(\varepsilon) \breve{\tau}_{\circ}}\right]\right)^{\frac{\varepsilon}{1+\varepsilon}}\left(\mathbb{E}_{x}\left[\mathrm{e}^{\left.\int_{0}^{\breve{\tau}_{\circ}}\left[(1+\varepsilon) f\left(X_{t}\right)-\Lambda((1+\varepsilon) f)\right] \mathrm{d} t\right]}\right)^{\frac{1}{1+\varepsilon}} .\right.
$$

Let $\Psi_{\epsilon}$ denote a positive solution of (2.3) for $(1+\varepsilon) f$ and $\Lambda((1+\varepsilon) f)$. Then by Fatou's lemma we have

$$
\mathbb{E}_{x}\left[\mathrm{e}^{\mathrm{f}_{0}^{\check{\tau}_{\circ}}\left[(1+\varepsilon) f\left(X_{t}\right)-\Lambda((1+\varepsilon) f)\right] \mathrm{d} t}\right] \leq \Psi_{\varepsilon}(x)\left(\inf _{\partial \mathcal{B} \circ} \Psi_{\varepsilon}\right)^{-1} \quad \forall x \in \mathcal{B}_{\circ}^{c} .
$$

The claim then follows by (2.47), (2.48), and (2.49).

Let $\varepsilon>0$ be as in (H3) and $\Psi_{\varepsilon}$ denote the solution of the MPE for $(1+\varepsilon) f$. It is straightforward to show using Hölder's inequality together with the stochastic representation equation in 2.41) that $F:=\Psi_{\varepsilon} \Psi^{-1}$ is inf-compact. Indeed if $\varepsilon f$ is near monotone relative to $\Lambda((1+\varepsilon) f)-\Lambda(f)$, this implies that for some ball $\mathcal{B}$ and constants $\delta>0$ and $\gamma>0$ we have

$$
\begin{aligned}
f-\Lambda(f) & \geq \delta \\
(1+\varepsilon) f-\Lambda((1+\varepsilon) f) & \geq(1+\gamma)(f-\Lambda(f))
\end{aligned}
$$

on $\mathcal{B}^{c}$, where we also use the property that $f$ is near monotone relative to $\Lambda(f)$. By (2.50) and Jensen's inequality, and letting $\breve{\tau}=\breve{\tau}\left(\mathcal{B}^{c}\right)$, we obtain

$$
\left(\mathbb{E}_{x}\left[\mathrm{e}^{\int_{0}^{\breve{\tau}}\left[f\left(X_{t}\right)-\Lambda(f)\right] \mathrm{d} t}\right]\right)^{1+\gamma} \leq \mathbb{E}_{x}\left[\mathrm{e}^{\int_{0}^{\breve{\tau}}\left[(1+\varepsilon) f\left(X_{t}\right)-\Lambda((1+\varepsilon) f)\right] \mathrm{d} t}\right] \quad \forall x \in \mathcal{B}^{c} .
$$

Therefore, by (2.41) and (2.51) we have

$$
\frac{\Psi_{\varepsilon}(x)}{\Psi(x)} \geq M_{\mathcal{B}}(\Psi(x))^{\gamma} \quad \forall x \in \mathcal{B}^{c}
$$

with

$$
M_{\mathcal{B}}:=\left(\inf _{\partial \mathcal{B}} \Psi_{\varepsilon}\right)\left(\sup _{\partial \mathcal{B}} \Psi\right)^{-1-\gamma}
$$

By (2.4) and (2.50) we obtain

$$
\begin{aligned}
\mathcal{L}^{*} F(x) & =(\Lambda((1+\varepsilon) f)-\Lambda(f)-\varepsilon f) F(x) \\
& \leq-\gamma(f-\Lambda(f)) F(x) \\
& \leq-\gamma \delta F(x) \quad \forall x \in \mathcal{B}^{c} .
\end{aligned}
$$

The Foster-Lyapunov equation in (2.53) implies of course that the diffusion is geometrically ergodic. The estimate in (1.25) is obtained as the one in (2.1) using (2.52) and (2.53). 


\section{Proofs of the results on the control problem}

For the proof of Proposition 1.1 we need some auxiliary lemmas. The lemma which follows is the nonlinear Dirichlet eigenvalue problem studied in [46], combined with a result from [11, Lemma 2.1].

Lemma 3.1. For each $n \in \mathbb{N}$, there exists a unique pair $\left(\widehat{V}_{n}, \hat{\lambda}_{n}\right) \in\left(C^{2}\left(B_{n}\right) \cap \mathcal{C}\left(\bar{B}_{n}\right)\right) \times \mathbb{R}$, satisfying $\widehat{V}_{n}>0$ on $B_{n}, \widehat{V}_{n}=0$ on $\partial B_{n}$, and $\widehat{V}_{n}(0)=1$, which solves

$$
\min _{u \in \mathbb{U}}\left[\mathcal{L}^{u} \widehat{V}_{n}(x)+c(x, u) \widehat{V}_{n}(x)\right]=\hat{\lambda}_{n} \widehat{V}_{n}(x), \quad x \in B_{n} .
$$

Moreover, $\hat{\lambda}_{n}<\hat{\lambda}_{n+1}<\Lambda^{*}$ for all $n \in \mathbb{N}$.

Let us add here that, as shown in [46], the non-linear elliptic operator in Lemma 3.1 has two principal eigenvalues. In the setting of [46], $-\hat{\lambda}_{n}$ is the principal eigenvalue in $B_{n}$ with negative principal eigenfunction $-\widehat{V}_{n}$. Then strict monotonicity of $\hat{\lambda}_{n}$ follows from [46, Remark 3] (or by the strong maximum principle).

Lemma 3.2. Suppose that $\sigma$ is bounded and

$$
\max _{u \in \mathbb{U}} \frac{\langle b(x, u), x\rangle^{+}}{|x|} \underset{|x| \rightarrow \infty}{\longrightarrow} 0 .
$$

Then,

$$
\limsup _{t \rightarrow \infty} \frac{1}{t} \mathbb{E}_{x}^{U}\left[\left|X_{t}\right|\right]=0 \quad \forall U \in \mathfrak{U} .
$$

Proof. We claim that for each $\varepsilon>0$ there exists a positive constant $C_{\varepsilon}$ such that $\varepsilon C_{\varepsilon} \rightarrow 0$, as $\varepsilon \searrow 0$, and

$$
\max _{u \in \mathbb{U}}\langle b(x, u), x\rangle^{+} \leq C_{\varepsilon}(1+\varepsilon|x|) \quad \forall x \in \mathbb{R}^{d} .
$$

Indeed, if $f$ is nonnegative and $f(x) \in \mathfrak{o}(|x|)$, we write

$$
\begin{aligned}
f(x) & \leq \sup _{|x|<R} f(x)+\left(\sup _{|x| \geq R} \frac{f(x)}{|x|}\right)|x| \\
& =M_{R}+\varepsilon_{R}|x| \\
& <1+M_{R}+\varepsilon_{R}|x| \\
& =\left(1+M_{R}\right)\left(1+\frac{\varepsilon_{R}}{1+M_{R}}|x|\right),
\end{aligned}
$$

which proves the claim since $\varepsilon_{R} \searrow 0$ as $R \nearrow \infty$.

Applying Itô's formula in (1.3), under any control $U \in \mathfrak{U}$, we have

$$
\begin{aligned}
\mathbb{E}_{x}^{U}\left[\left|X_{t}\right|^{2}\right] & \leq|x|^{2}+\int_{0}^{t} \mathbb{E}_{x}^{U}\left[2\left\langle b\left(X_{s}, U_{s}\right), X_{s}\right\rangle^{+}+\operatorname{trace}\left(a\left(X_{s}\right)\right)\right] \mathrm{d} s \\
& \leq|x|^{2}+C_{\varepsilon}^{\prime} \int_{0}^{t}\left(1+\varepsilon \mathbb{E}_{x}^{U}\left[\left|X_{s}\right|\right]\right) \mathrm{d} s,
\end{aligned}
$$

where $C_{\varepsilon}^{\prime}$ also satisfies $\varepsilon C_{\varepsilon}^{\prime} \rightarrow 0$, as $\varepsilon \searrow 0$. Let $\varphi(t)$ denote the right hand side of (3.1). Then

$$
\dot{\varphi}(t) \leq C_{\varepsilon}^{\prime}(1+\varepsilon \sqrt{\varphi(t)}),
$$

which implies that

$$
\frac{\dot{\varphi}(t)}{\sqrt{\varepsilon^{-2}+\varphi(t)}} \leq 2 \varepsilon C_{\varepsilon}^{\prime}
$$


Integrating (3.2), we have $\sqrt{\varepsilon^{-2}+\varphi(t)} \leq \varepsilon C_{\varepsilon}^{\prime} t+\sqrt{\varepsilon^{-2}+|x|^{2}}$ and using (3.1), we obtain

$$
\begin{aligned}
\mathbb{E}_{x}^{U}\left[\left|X_{t}\right|\right] & \leq \sqrt{\varphi(t)} \\
& \leq \sqrt{\varepsilon^{-2}+\varphi(t)} \leq \varepsilon C_{\varepsilon}^{\prime} t+\sqrt{\varepsilon^{-2}+|x|^{2}} .
\end{aligned}
$$

Since (3.3) holds for all $\varepsilon>0$, the result follows.

We need the following lemma.

Lemma 3.3. Let $\varphi \in \mathcal{W}_{\mathrm{loc}}^{2, p}, p>d$, be a strong, positive solution of

$$
\mathcal{L} \varphi+h \varphi=0
$$

where $h \in L^{\infty}\left(\mathbb{R}^{d}\right)$. Suppose that $b$ and $\sigma$ are bounded and $\sigma$ is Lipschitz continuous. Then there exists a constant $\tilde{C}$ such that

$$
\sup _{x \in \mathbb{R}^{d}} \frac{|\nabla \varphi(x)|}{\varphi(x)}<\tilde{C} .
$$

Proof. Let $x \in \mathbb{R}^{d}$. By (1.30) we obtain

$$
\varphi(y) \leq C_{H} \varphi(z) \text { for all } z, y \in B_{2}(x) .
$$

Since the coefficients $b, \sigma$ and $h$ are bounded, the Harnack constant $C_{H}$ in (3.4) does not depend on $x$. Again, applying (1.31) and the Sobolev embedding theorem mentioned in the beginning of Section 3 . we obtain

$$
\sup _{y \in B_{1}(x)}|\nabla \varphi(y)| \leq \tilde{C} \sup _{z \in B_{2}(x)} \varphi(z) \leq \tilde{C} C_{H} \inf _{z \in B_{2}(x)} \varphi(z) \leq \tilde{C} C_{H} \varphi(x),
$$

where the constant $\tilde{C}$ does not depend on $x$. Hence the result follows.

Theorem 3.4. Suppose Assumption 1.1 and (1.5) hold, and that $c$ is near-monotone relative to $\Lambda^{*}$ and bounded. Then

(i) There exists a solution $\left(V^{*}, \Lambda^{*}\right) \in \mathcal{C}^{2}\left(\mathbb{R}^{d}\right) \times \mathbb{R}$, satisfying $V^{*}(0)=1$ and $\inf _{\mathbb{R}^{d}} V^{*}>0$, to the HJB equation

$$
\min _{u \in \mathbb{U}}\left[\mathcal{L}^{u} V^{*}(x)+c(x, u) V^{*}(x)\right]=\Lambda^{*} V^{*}(x) \quad \forall x \in \mathbb{R}^{d} .
$$

(ii) If $(V, \Lambda) \in \mathfrak{V}$ satisfies

$$
\min _{u \in \mathbb{U}}\left[\mathcal{L}^{u} V(x)+c(x, u) V(x)\right]=\Lambda V(x) \quad \forall x \in \mathbb{R}^{d},
$$

then $\Lambda=\Lambda^{*}$, and $\inf _{\mathbb{R}^{d}} V>0$. In addition, any measurable selector from the minimizer of (3.5) belongs to $\mathfrak{U}_{\mathrm{SSM}}$ and is optimal, i.e., $\Lambda_{x}^{v}=\Lambda^{*}$ for all $x \in \mathbb{R}^{d}$.

Proof. As shown in [11, Lemma 2.1], any limit point, $\left(V^{*}, \lambda^{*}\right) \in \mathcal{C}^{2}\left(\mathbb{R}^{d}\right) \times \mathbb{R}$ of the eigensolutions $\left(\widehat{V}_{n}, \hat{\lambda}_{n}\right)$ of the Dirichlet problem on $B_{n}$ in Lemma 3.1] as $n \rightarrow \infty$, satisfies

$$
\min _{u \in \mathbb{U}}\left[\mathcal{L}^{u} V^{*}(x)+c(x, u) V^{*}(x)\right]=\lambda^{*} V^{*}(x) \quad \forall x \in \mathbb{R}^{d} .
$$

Clearly then, $V^{*}>0$ on $\mathbb{R}^{d}, V^{*}(0)=1$, and $\lambda^{*} \leq \Lambda^{*}$ by Lemma 3.1 Since $b, \sigma$, and $c$ are bounded, and $\nabla\left(\log V^{*}\right)=\frac{\nabla V^{*}}{V^{*}}$, then by Lemma 3.3 there exists a constant $\kappa>0$ such that

$$
\mathrm{e}^{-\kappa(1+|x|)} \leq V^{*}(x) \leq \mathrm{e}^{\kappa(1+|x|)} \quad \forall x \in \mathbb{R}^{d}
$$


Let $v$ be a measurable selector from the minimizer of (3.6). A straightforward application of Fatou's lemma on the stochastic representation of the solution $V^{*}$ of (3.6) shows that

$$
V^{*}(x) \geq \mathbb{E}_{x}^{v}\left[\mathrm{e}^{\int_{0}^{T}\left[c\left(X_{t}, v\left(X_{t}\right)\right)-\lambda^{*}\right] \mathrm{d} t} V^{*}\left(X_{T}\right)\right] \quad \forall T>0 .
$$

Evaluating (3.8) at $x=0$, taking the logarithm on both sides, applying Jensen's inequality, dividing by $T$, and rearranging terms, we obtain

$$
\frac{1}{T} \mathbb{E}_{x}^{v}\left[\int_{0}^{T} c\left(X_{t}, v\left(X_{t}\right)\right) \mathrm{d} t\right]+\frac{1}{T} \mathbb{E}_{x}^{v}\left[\log V^{*}\left(X_{T}\right)\right] \leq \lambda^{*}+\frac{1}{T} \log V^{*}(x) .
$$

Hence, since $\left|\log V^{*}\left(X_{T}\right)\right| \leq \kappa\left(1+\left|X_{T}\right|\right)$ by (3.7), it follows by Lemma 3.2 that

$$
\limsup _{T \rightarrow \infty} \frac{1}{T} \mathbb{E}_{x}^{v}\left[\left|\log V^{*}\left(X_{T}\right)\right|\right]=0 .
$$

Therefore, by (3.9) we obtain

$$
\limsup _{T \rightarrow \infty} \frac{1}{T} \mathbb{E}_{x}^{v}\left[\int_{0}^{T} c\left(X_{t}, v\left(X_{t}\right)\right) \mathrm{d} t\right] \leq \lambda^{*}
$$

Since $\lambda^{*} \leq \Lambda^{*}$, it follows that $v$ is stable by the argument used in the proof of $(\mathrm{g}) \Rightarrow$ (a) in Lemma 2.1 Also $V^{*}$ is inf-compact, and $\Lambda^{v}(c) \leq \lambda^{*}$ by Lemma 2.1(d), and (f), respectively. Thus we have shown that

$$
\Lambda_{x}^{v}(c) \leq \lambda^{*} \leq \Lambda^{*} \quad \forall x \in \mathbb{R}^{d} .
$$

Therefore, we have $\lambda^{*}=\Lambda^{*}=\Lambda_{x}^{v}(c)$ for all $x \in \mathbb{R}^{d}$ by the definition of $\Lambda^{*}$. This proves part (i).

The proof of parts (ii) follows by the same arguments as in the preceding paragraph.

In order to simplify the notation, we define

$$
\bar{c}_{v}(x):=c(x, v(x)) \quad \forall x \in \mathbb{R}^{d}, \quad v \in \mathfrak{U}_{\mathrm{SM}} .
$$

Also recall that

$$
\mathfrak{U}_{\mathrm{SM}}^{*}:=\left\{v \in \mathfrak{U}_{\mathrm{SM}}: \Lambda_{x}^{v}(c)=\Lambda_{\mathrm{m}}^{*}, \forall x \in \mathbb{R}^{d}\right\} .
$$

The proof in Lemma 2.1 (f) $\Rightarrow$ (a), shows that any $v \in \mathfrak{U}_{\mathrm{SM}}^{*}$ is a stable Markov control.

In the two lemmas which follow we do not impose Assumption 1.1 .

Lemma 3.5. Suppose that $c$ is near-monotone relative to $\Lambda_{\mathrm{m}}^{*}$, and that $V \in \mathcal{C}^{2}\left(\mathbb{R}^{d}\right)$ is a positive solution of

$$
\min _{u \in \mathbb{U}}\left[\mathcal{L}^{u} V(x)+c(x, u) V(x)\right]=\Lambda_{\mathrm{m}}^{*} V(x) \quad \forall x \in \mathbb{R}^{d} .
$$

Let $v \in \mathfrak{U}_{\mathrm{SM}}^{*}$, and $V_{v} \in \mathcal{W}_{\mathrm{loc}}^{2, p}\left(\mathbb{R}^{d}\right), p>d$, be a positive solution of

$$
\mathcal{L}_{v} V_{v}(x)+\bar{c}_{v}(x) V_{v}(x)=\Lambda_{\mathrm{m}}^{*} V_{v}(x) \quad \text { a.e. in } \mathbb{R}^{d} .
$$

If for some ball $\mathcal{B} \in \mathbb{R}^{d}$ it holds that

$$
V(x) \leq \mathbb{E}_{x}^{v}\left[\mathrm{e}^{\mathrm{e}_{0}^{*}\left[\bar{c}_{v}\left(X_{s}\right)-\Lambda_{\mathrm{m}}^{*}\right] \mathrm{d} s} V\left(X_{\breve{\tau}}\right)\right] \quad \forall x \in \mathcal{B}^{c},
$$

with $\breve{\tau}=\tau\left(\mathcal{B}^{c}\right)$, then $\frac{V}{V_{v}}$ is constant on $\mathbb{R}^{d}$. In particular,

$$
\mathcal{L}_{v} V(x)+\bar{c}_{v}(x) V(x)=\Lambda_{\mathrm{m}}^{*} V(x) \quad \text { a.e. in } \mathbb{R}^{d} .
$$


Proof. By Itô's formula and the Fatou lemma, we have

$$
V_{v}(x) \geq \mathbb{E}_{x}^{v}\left[\mathrm{e}^{\int_{0}^{\breve{\tau}}\left[\bar{c}_{v}\left(X_{s}\right)-\Lambda_{\mathrm{m}}^{*}\right] \mathrm{d} s} V_{v}\left(X_{\breve{\tau}}\right)\right] \quad \forall x \in \mathcal{B}^{c}
$$

Therefore we can scale $V_{v}$, by multiplying with a positive constant, so that it touches $V$ from above at some point in $\mathcal{B}$. Since (3.10) implies that

$$
\mathcal{L}_{v} V(x)+\bar{c}_{v}(x) V(x) \geq \Lambda_{\mathrm{m}}^{*} V(x) \quad \text { a.e. in } \mathbb{R}^{d},
$$

and thus,

$$
\mathcal{L}_{v}\left(V_{v}-V\right)(x)-\left(\bar{c}_{v}(x)-\Lambda_{\mathrm{m}}^{*}\right)^{-}\left(V_{v}-V\right)(x) \leq 0 \quad \text { a.e. in } \mathbb{R}^{d},
$$

the result follows by the strong maximum principle.

Lemma 3.6. Let $c$ be near-monotone relative to $\Lambda_{\mathrm{m}}^{*}$, and suppose that $\Lambda_{\mathrm{m}}^{*}(c+h)>\Lambda_{\mathrm{m}}^{*}(c)$ for all $h \in \mathcal{C}_{\circ}$. Then for any positive solution $V_{v} \in \mathcal{W}_{\mathrm{loc}}^{2, p}\left(\mathbb{R}^{d}\right), p>d$, of (3.11), with $v \in \mathfrak{U}_{\mathrm{SM}}^{*}$, it holds that

$$
\min _{u \in \mathbb{U}}\left[\mathcal{L}^{u} V_{v}(x)+c(x, u) V_{v}(x)\right]=\Lambda_{\mathrm{m}}^{*} V_{v}(x) \quad \forall x \in \mathbb{R}^{d} .
$$

Proof. Let $\tilde{v}$ be a minimizing selector of

$$
x \mapsto \underset{u \in \mathbb{U}}{\arg \min }\left\{\left\langle b(x, u), \nabla V_{v}(x)\right\rangle+c_{v}(x) V_{v}(x)\right\} .
$$

Then $\mathcal{L}_{\tilde{v}} V_{v}(x)+c_{\tilde{v}}(x) V_{v}(x) \leq \Lambda_{\mathrm{m}}^{*} V_{v}$ which implies that $\Lambda^{\tilde{v}}(c) \leq \Lambda_{\mathrm{m}}^{*}$, and thus $\tilde{v} \in \mathfrak{U}_{\mathrm{SM}}^{*}$ and $\Lambda^{\tilde{v}}(c)=\Lambda_{\mathrm{m}}^{*}$. If (3.12) is not an equality, then for some measurable set $A$ of positive Lebesgue measure we have

$$
\mathcal{L}_{\tilde{v}} V_{v}+\left(\bar{c}_{\tilde{v}}+\mathbb{1}_{A}\right) V_{v} \leq \Lambda_{\mathrm{m}}^{*} V_{v} \quad \text { a.e. in } \mathbb{R}^{d} .
$$

Thus $\Lambda^{\tilde{v}}\left(c+\mathbb{1}_{A}\right) \leq \Lambda_{\mathrm{m}}^{*}$ by Lemma 2.1(f). However, if (1.8) holds, then by Theorem 1.5 we have

$$
\Lambda^{\tilde{v}}\left(c+\mathbb{1}_{A}\right)>\Lambda^{\tilde{v}}(c)=\Lambda_{\mathrm{m}}^{*},
$$

which is a contradiction. Thus (3.12) holds.

Remark 3.1. Let $\mathcal{V}:=\left\{V \in \mathcal{C}^{2}\left(\mathbb{R}^{d}\right): V(0)=1, \inf _{\mathbb{R}^{d}} V_{v}>0\right\}$, and

$$
\begin{aligned}
& \overline{\mathfrak{G}}:=\{V \in \mathcal{V}: V \text { solves }[3.10) \\
& \mathfrak{G}:=\left\{V_{v} \in \mathcal{V}: V_{v} \text { solves (3.11) for some } v \in \mathfrak{U}_{\mathrm{SM}}^{*}\right\} .
\end{aligned}
$$

It follows by Lemma 3.6 that, if $\Lambda_{\mathrm{m}}^{*}(c+h)>\Lambda_{\mathrm{m}}^{*}(c)$ for all $h \in \mathcal{C}_{\text {o }}$, then $\overline{\mathfrak{G}}=\mathfrak{G}$.

We continue with the proof of Theorem 1.2

Proof of Theorem 1.2. In view of Theorem 3.4(ii) it suffices to prove uniqueness in the class $\mathfrak{V}_{\circ}$. Also recall that $\Lambda^{*}=\Lambda_{\mathrm{m}}^{*}$. Since the Dirichlet eigenvalues in Lemma 3.1 satisfy $\hat{\lambda}_{n}<\Lambda^{*}$ for all $n \in \mathbb{N}$, the Dirichlet problem

$$
\min _{u \in \mathbb{U}}\left[\mathcal{L}^{u} \varphi_{n}(x)+\left(c(x, u)-\Lambda^{*}\right) \varphi_{n}(x)\right]=-\alpha_{n} \mathbb{1}_{\mathcal{B}}(x) \quad \text { a.e. } x \in B_{n}, \quad \varphi_{n}=0 \text { on } \partial B_{n},
$$

with $\alpha_{n}>0$, has a unique solution $\varphi_{n} \in \mathcal{W}_{\text {loc }}^{2, p}\left(B_{n}\right) \cap \mathcal{C}\left(\bar{B}_{n}\right)$, for any $p \geq 1$ [46, Theorem 1.9] (see also [52, Theorem 1.1(ii)]). We choose $\alpha_{n}$ as in Lemma 2.11. Namely if $\tilde{\alpha}_{n}>0$ is such that the solution $\varphi_{n}$ of (3.13) with $\alpha_{n}=\tilde{\alpha}_{n}$ satisfies $\varphi_{n}(0)=1$, we set $\alpha_{n}=\min \left(1, \tilde{\alpha}_{n}\right)$. Passing to the limit to obtain a positive solution $\Phi \in \mathcal{W}_{\mathrm{loc}}^{2, p}\left(\mathbb{R}^{d}\right)$ of

$$
\min _{u \in \mathbb{U}}\left[\mathcal{L}^{u} \Phi(x)+\left(c(x, u)-\Lambda^{*}\right) \Phi(x)\right]=-\alpha \mathbb{1}_{\mathcal{B}}(x), \quad x \in \mathbb{R}^{d}
$$


We claim that $\inf _{\mathbb{R}^{d}} \Phi>0$. Indeed, let $\hat{v}$ be a measurable selector from the minimizer of (3.14). Then

$$
\mathcal{L}_{\hat{v}} \Phi(x)+\left(\bar{c}_{\hat{v}}(x)-\Lambda^{*}\right) \Phi(x)=-\alpha \mathbb{1}_{\mathcal{B}}(x), \quad \text { a.e. in } \mathbb{R}^{d} .
$$

Consider a ball $\breve{\mathcal{B}}$ which is concentric with $\mathcal{B}$ and of twice the radius. By the proof of Lemma 3.3 we obtain

$$
\sup _{x \in \breve{\mathcal{B}}^{c}} \frac{|\nabla \Phi(x)|}{\Phi(x)}<\breve{C},
$$

for some constant $\breve{C}$. Since $\inf _{\breve{B}} \Phi>0$, it follows that $\frac{|\nabla \Phi(x)|}{\Phi(x)}$ is bounded on $\mathbb{R}^{d}$. Hence a bound as in (3.7) holds for $\Phi$. By Itô's formula and Fatou's lemma we obtain from (3.15) that

$$
\Phi(x) \geq \mathbb{E}_{x}^{v}\left[\mathrm{e}^{\int_{0}^{T}\left[\bar{c}_{\hat{v}}\left(X_{t}\right)-\Lambda^{*}\right] \mathrm{d} t} \Phi\left(X_{T}\right)\right] \quad \forall T>0
$$

and then proceed exactly as in the proof of Theorem 3.4 to show that $\Phi$ is inf-compact. This proves the claim.

Let $v$ be any control in $\mathfrak{U}_{\mathrm{SM}}^{*}$. By Itô's formula applied to (3.13), with $\breve{\tau}=\tau\left(\mathcal{B}^{c}\right)$, we obtain

$$
\begin{aligned}
\varphi_{n}(x) \leq \mathbb{E}_{x}\left[\mathrm{e}^{\int_{0}^{\breve{\tau}}\left[\bar{c}_{v}\left(X_{s}\right)-\Lambda_{\mathrm{m}}^{*}\right] \mathrm{d} s} \varphi_{n}\left(X_{\breve{\tau})} \mathbb{1}_{\left\{\breve{\tau}<T \wedge \tau_{n}\right\}}\right]\right. & \\
& +\mathbb{E}_{x}\left[\mathrm{e}^{\int_{0}^{T}\left[\bar{c}_{v}\left(X_{s}\right)-\Lambda_{\mathrm{m}}^{*}\right] \mathrm{d} s} \varphi_{n}\left(X_{T}\right) \mathbb{1}_{\left\{T<\breve{\tau} \wedge \tau_{n}\right\}}\right] \quad \forall x \in B_{n} \backslash \mathcal{B}, \forall T>0 .
\end{aligned}
$$

Then we use the argument in the proof of Lemma 2.11, by replacing $f$ with $\bar{c}_{v}$, and $\Lambda(f)$ with $\Lambda_{\mathrm{m}}^{*}$ in (2.34) - 2.36) to obtain

$$
\Phi(x) \leq \mathbb{E}_{x}\left[\mathrm{e}^{\int_{0}^{\breve{\tau}}\left[\bar{c}_{v}\left(X_{s}\right)-\Lambda^{*}\right] \mathrm{d} s} \Phi\left(X_{\breve{\tau})}\right] \quad \forall x \in \mathcal{B}^{c},\right.
$$

for all $v \in \mathfrak{U}_{\mathrm{SM}}^{*}$.

Using (3.15) and the assumption $\Lambda_{\mathrm{m}}^{*}(c+h) \geq \Lambda_{\mathrm{m}}^{*}(c)$ for all $h \in \mathcal{C}_{\circ}$, we conclude as in the proof of Lemma 2.11 that $\alpha=0$. Recall the definition of $\overline{\mathfrak{G}}$ and $\mathfrak{G}$ in Remark 3.1. Thus by Lemma 3.5] we obtain $\mathfrak{G}=\{\Phi\}$, and since $\overline{\mathfrak{G}}=\mathfrak{G}$ by Remark 3.1, uniqueness of the solution to (1.9) and the verification part of the result follow.

Since by Fatou's lemma the converse inequality to (3.16) holds, the representation in (1.10) follows by Theorem 1.5 .

For the converse, note that $\Lambda_{\mathrm{m}}^{*}(c+h)=\Lambda_{\mathrm{m}}^{*}(c)$ implies the existence of $v \in \mathfrak{U}_{\mathrm{SM}}$ such that

$$
\Lambda_{x}^{v}(c+h)=\Lambda_{\mathrm{m}}^{*}(c+h)=\Lambda_{\mathrm{m}}^{*}(c) \leq \Lambda_{x}^{v}(c)
$$

for all $x \in \mathbb{R}^{d}$. But $\Lambda_{x}^{v}(c+h) \geq \Lambda_{x}^{v}(c)$. Therefore, $v \in \mathfrak{U}_{\mathrm{SM}}^{*}$ and $\Lambda_{x}^{v}(c+h)=\Lambda_{x}^{v}(c)$. If this is the case, then (1.10) contradicts Theorem 1.5. This completes the proof.

Proof of Proposition 1.3. Let $\varepsilon_{0}>0$ be small enough so that $c$ is near-monotone relative to $\Lambda_{\mathrm{m}}^{*}+\varepsilon_{0}$, and for $\varepsilon \in\left(0, \varepsilon_{0}\right)$, let $v_{\varepsilon} \in \mathfrak{U}_{\mathrm{SM}}$ be a $\varepsilon$-optimal control relative to $\Lambda_{\mathrm{m}}^{*}$. In other words, $v_{\varepsilon}$ satisfies $\Lambda^{v_{\varepsilon}} \leq \Lambda_{\mathrm{m}}^{*}+\varepsilon$. An analogous argument as in the proof $(\mathrm{g}) \Rightarrow(\mathrm{a})$ of Lemma 2.1 shows that $v_{\varepsilon}$ is a stable Markov control. Define

$$
b_{n}^{v_{\varepsilon}}(x, u):= \begin{cases}b(x, u) & \text { for } x \in B_{n}, \quad u \in \mathbb{U}, \\ b\left(x, v_{\varepsilon}(x)\right) & \text { for } x \in B_{n}^{c}, \quad u \in \mathbb{U},\end{cases}
$$

and $c_{n}^{v_{\varepsilon}}(x, u)$ in an exactly analogous manner. Let $\mathfrak{U}_{\mathrm{SM}}^{n, v_{\varepsilon}}$ denote the class of stationary Markov controls that agree with $v_{\varepsilon}$ on $B_{n}^{c}$. By [46, Theorem 1.1], there exists a unique pair $\left(V_{n, k}^{v_{\varepsilon}}, \lambda_{n, k}^{v_{\varepsilon}}\right) \in\left(\mathcal{W}^{2, p}\left(B_{k}\right) \cap \mathcal{C}\left(\bar{B}_{k}\right)\right) \times \mathbb{R}$, for any $p>d$, satisfying $V_{n, k}^{v_{\varepsilon}}>0$ on $B_{k}$ and $V_{n, k}^{v_{\varepsilon}}(0)=1$, which solves

$$
\min _{u \in \mathbb{U}}\left[\mathcal{L}^{u} V_{n, k}^{v_{\varepsilon}}(x)+c_{n}^{v_{\varepsilon}}(x, u) V_{n, k}^{v_{\varepsilon}}(x)\right]=\lambda_{n, k}^{v_{\varepsilon}} V_{n, k}^{v_{\varepsilon}}(x) \quad \text { a.e. } x \in B_{k},
$$


and $V_{n, k}^{v_{\varepsilon}}=0$ on $\partial B_{k}$ (compare with Lemma 3.1). Following the proof of [11, Lemma 2.1], we deduce that $\lambda_{n, k}^{v_{\varepsilon}} \leq \Lambda_{x}^{v_{\varepsilon}}$ for all $x \in B_{k}$. Since $V_{n, k}^{v_{\varepsilon}}$ is locally bounded, uniformly in $k \in \mathbb{N}$, by Harnack's inequality, we have $\sup _{k}\left\|V_{n, k}^{v_{\varepsilon}}\right\|_{\mathcal{W}^{2, p}\left(B_{R}\right)}<\infty$ for all $R>0$, and any $p>d$. Thus taking limits as $k \rightarrow \infty$ along some subsequence, we obtain by (3.17) a pair $\left(V_{n}^{v_{\varepsilon}}, \lambda_{n}^{v_{\varepsilon}}\right) \in \mathcal{W}_{\text {loc }}^{2, p}\left(\mathbb{R}^{d}\right) \times \mathbb{R}$, for any $p>d$, satisfying $\lambda_{n}^{v_{\varepsilon}} \leq \Lambda^{v_{\varepsilon}}$, $V_{n}^{v_{\varepsilon}}>0$ on $\mathbb{R}^{d}$, and $V_{n}^{v_{\varepsilon}}(0)=1$, which solves

$$
\min _{u \in \mathbb{U}}\left[\mathcal{L}^{u} V_{n}^{v_{\varepsilon}}(x)+c_{n}^{v_{\varepsilon}}(x, u) V_{n}^{v_{\varepsilon}}(x)\right]=\lambda_{n}^{v_{\varepsilon}} V_{n}^{v_{\varepsilon}}(x) \quad \text { a.e. } x \in \mathbb{R}^{d} .
$$

It also follows by elliptic regularity that the restriction of $V_{n}^{v_{\varepsilon}}$ in $B_{n}$ is in $\mathcal{C}^{2}\left(\mathbb{R}^{d}\right)$.

Let $\hat{v}_{n} \in \mathfrak{U}_{\mathrm{SM}}^{n, v_{\varepsilon}}$ be a measurable selector from the minimizer of (3.18). Since $\hat{v}_{n}$ agrees with $v_{\varepsilon}$ on $B_{n}^{c}$, any Lyapunov function for the diffusion under the control $v_{\varepsilon}$ is also a Lyapunov function under the control $\hat{v}_{n}$. It follows that $\hat{v}_{n} \in \mathfrak{U}_{\mathrm{SSM}}$. Let $\mathcal{B}$ be a bounded open ball such that $c(x, u)>\Lambda_{\mathrm{m}}^{*}+\varepsilon_{0}$ for all $(x, u) \in \mathcal{B}^{c} \times \mathbb{U}$. Applying Itô's formula to (3.18), and using Fatou's lemma, we obtain, with $\breve{\tau} \equiv \tau\left(\mathcal{B}^{c}\right)$, that

$$
\begin{aligned}
V_{n}^{v_{\varepsilon}}(x) & \geq \mathbb{E}_{x}^{\hat{v}_{n}}\left[\mathrm{e}^{\int_{0}^{\breve{\tau}}\left[c_{n}^{v_{\varepsilon}}\left(X_{t}, \hat{v}_{n}\left(X_{t}\right)\right)-\lambda_{n}^{v_{\varepsilon}}\right] \mathrm{d} t} V_{n}^{v_{\varepsilon}}\left(X_{\breve{\tau}}\right)\right] \\
& \geq\left(\min _{\partial \mathcal{B}\left(\varepsilon_{0}\right)} V_{n}^{v_{\varepsilon}}\right) \mathbb{E}_{x}^{\hat{v}_{n}}\left[\exp \left(\frac{1}{2}\left(\varepsilon_{0}-\varepsilon\right) \breve{\tau}\right)\right] \quad \forall x \in \mathcal{B}^{c} .
\end{aligned}
$$

Since $V_{n}^{v_{\varepsilon}}(0)=1$, it follows by the Harnack inequality that $\min _{\partial \mathcal{B}\left(\varepsilon_{0}\right)} V_{n}^{v_{\varepsilon}} \geq C_{H}^{-1}$. Therefore, by (3.19), we have

$$
\inf _{n \in \mathbb{N}} \inf _{\mathbb{R}^{d}} V_{n}^{v_{\varepsilon}}>0
$$

Again $\sup _{n}\left\|V_{n}^{v_{\varepsilon}}\right\|_{\mathcal{W}^{2, p}\left(B_{R}\right)}<\infty$ for all $R>0$, and any $p>d$. Thus, taking limits in (3.19) as $n \rightarrow \infty$, along some subsequence, we obtain a pair $\left(V^{v_{\varepsilon}}, \lambda^{v_{\varepsilon}}\right) \in \mathcal{C}^{2}\left(\mathbb{R}^{d}\right) \times \mathbb{R}$, satisfying $\lambda^{v_{\varepsilon}} \leq \Lambda^{v_{\varepsilon}}$, inf $\mathbb{R}^{d} V^{v_{\varepsilon}}>0$, and $V^{v_{\varepsilon}}(0)=1$, which solves

$$
\min _{u \in \mathbb{U}}\left[\mathcal{L}^{u} V^{v_{\varepsilon}}(x)+c(x, u) V^{v_{\varepsilon}}(x)\right]=\lambda^{v_{\varepsilon}} V^{v_{\varepsilon}}(x) \quad \text { a.e. } x \in \mathbb{R}^{d} .
$$

Once more, taking any limit of (3.21) as $\varepsilon \searrow 0$ along some subsequence, we obtain a function $V^{*} \in \mathcal{C}^{2}\left(\mathbb{R}^{d}\right)$, satisfying

$$
\min _{u \in \mathbb{U}}\left[\mathcal{L}^{u} V^{*}(x)+c(x, u) V^{*}(x)\right]=\Lambda_{\mathrm{m}}^{*} V^{*}(x) \quad \forall x \in \mathbb{R}^{d} .
$$

It holds that $\inf _{\mathbb{R}^{d}} V^{*}>0$ by (3.20), and $V^{*}(0)=1$ by construction.

Let $v^{*}$ be a measurable selector from the minimizer of (3.22). Then $v^{*} \in \mathfrak{U}_{\mathrm{SSM}}$, and $\Lambda_{x}^{v^{*}}=\Lambda_{\mathrm{m}}^{*}$ for all $x \in \mathbb{R}^{d}$ by Lemma 2.1

Suppose that $\Lambda_{\mathrm{m}}^{*}(c+h)>\Lambda_{\mathrm{m}}^{*}(c)$ for all $h \in \mathcal{C}_{\circ}$. We follow a variation of the construction in the proof of Theorem 2.8, using [46, Theorem 1.9]. Let $\theta>0$ be any positive constant such that $c$ is near monotone relative to $\Lambda_{\mathrm{m}}^{*}+2 \theta$, and fix some $\alpha \in(0, \theta)$. Let $\zeta_{\alpha}>0$. As argued in the proof of Theorem 1.2 , the Dirichlet problem

$$
\min _{u \in \mathbb{U}}\left[\mathcal{L}^{u} \varphi_{\alpha, n}(x)+\left(c(x, u)-\Lambda_{\mathrm{m}}^{*}-\alpha\right) \varphi_{\alpha, n}(x)\right]=-\zeta_{\alpha} \quad \text { a.e. } x \in B_{n}, \quad \varphi_{\alpha}=0 \quad \text { on } \partial B_{n},
$$

has a unique nonnegative solution $\varphi_{\alpha, n} \in \mathcal{C}^{2}\left(B_{n}\right) \cap \mathcal{C}\left(\bar{B}_{n}\right)$. It is clear by the strong maximum principle that $\varphi_{\alpha, n}$ is positive in $B_{n}$. For $v \in \mathfrak{U}_{\mathrm{SM}}^{*}$, let $V_{v} \in \mathcal{W}_{\text {loc }}^{2, p}\left(\mathbb{R}^{d}\right), p>d, V_{v}(0)=1$, be a positive solution of

$$
\mathcal{L}_{v} V_{v}(x)+\bar{c}_{v}(x) V_{v}(x)=\Lambda_{\mathrm{m}}^{*} V_{v}(x) \quad \text { a.e. in } \mathbb{R}^{d} .
$$

Writing (3.24) as

$$
\mathcal{L}_{v} V_{v}(x)+\left(\bar{c}_{v}(x)-\Lambda_{\mathrm{m}}^{*}-\alpha\right) V_{v}(x)=-\alpha V_{v}(x) \quad \text { a.e. } x \in \mathbb{R}^{d},
$$


and using Itô's formula and Fatou's lemma we obtain

$$
V_{v}(x) \geq \mathbb{E}_{x}\left[\mathrm{e}^{\int_{0}^{\tau_{n}}\left[\bar{c}_{v}\left(X_{s}\right)-\Lambda_{\mathrm{m}}^{*}-\alpha\right] \mathrm{d} s} V_{v}\left(X_{\tau_{n}}\right)\right]+\alpha \mathbb{E}_{x}\left[\int_{0}^{\tau_{n}} \mathrm{e}^{\int_{0}^{t}\left[\bar{c}_{v}\left(X_{s}\right)-\Lambda_{\mathrm{m}}^{*}-\alpha\right] \mathrm{d} s} V_{v}\left(X_{t}\right) \mathrm{d} t\right]
$$

for any $\alpha \geq 0$, and we use this as in the proof of Theorem 2.8 to deduce that

$$
\varphi_{\alpha, n} \leq \frac{\zeta_{\alpha}}{\alpha}\left(\inf _{\mathbb{R}^{d}} V_{v}\right) V_{v} \quad \forall n \in \mathbb{N},
$$

and for all $v \in \mathfrak{U}_{\mathrm{SM}}^{*}$. As explained in Theorem 2.8 this allows us to pass to a limit along some sequence $n \rightarrow \infty$, to obtain a positive function $\Phi_{\alpha} \in \mathcal{C}^{2}\left(\mathbb{R}^{d}\right)$ which solves

$$
\min _{u \in \mathbb{U}}\left[\mathcal{L}^{u} \Phi_{\alpha}(x)+\left(c(x, u)-\Lambda_{\mathrm{m}}^{*}-\alpha\right) \Phi_{\alpha}(x)\right]=-\zeta_{\alpha} \quad \forall x \in \mathbb{R}^{d} .
$$

By Itô's formula we obtain from (3.23) that

$$
\begin{aligned}
\varphi_{\alpha, n}(x) \leq \mathbb{E}_{x}\left[\mathrm{e}^{\int_{0}^{\breve{\tau}_{r} \wedge T}\left[\bar{c}_{v}\left(X_{s}\right)-\Lambda_{\mathrm{m}}^{*}-\alpha\right] \mathrm{d} s} \varphi_{\alpha, n}\left(X_{\breve{\tau}_{r} \wedge T}\right) \mathbb{1}_{\left\{\breve{\tau}_{r} \wedge T<\tau_{n}\right\}}\right] \\
+\zeta_{\alpha} \mathbb{E}_{x}\left[\int_{0}^{\breve{\tau}_{r} \wedge T \wedge \tau_{n}} \mathrm{e}^{\int_{0}^{t}\left[\bar{c}_{v}\left(X_{s}\right)-\Lambda_{\mathrm{m}}^{*}-\alpha\right] \mathrm{d} s} \mathrm{~d} t\right] \quad \forall(T, x) \in \mathbb{R}_{+} \times\left(B_{n} \backslash B_{r}\right),
\end{aligned}
$$

and for all $v \in \mathfrak{U}_{\mathrm{SM}}^{*}$, where we use the property that $\varphi_{\alpha, n}=0$ on $\partial B_{n}$. Since

$$
\mathbb{E}_{x}\left[\mathrm{e}^{\mathrm{e}_{0}^{\tau_{r} \wedge T}\left[\bar{c}_{v}\left(X_{s}\right)-\Lambda_{\mathrm{m}}^{*}\right] \mathrm{d} s}\right] \leq\left(\inf _{\mathbb{R}^{d}} V_{v}\right)^{-1} V_{v}(x) \quad \forall x \in B_{n} \backslash B_{r}, \forall r, T>0,
$$

using dominated convergence for the first term on the right hand side of (3.26), and monotone convergence for the second term, we first take limits as $T \rightarrow \infty$, and then as $n \rightarrow \infty$ to obtain

$$
\Phi_{\alpha}(x) \leq \mathbb{E}_{x}^{v}\left[\mathrm{e}^{\int_{0}^{\ddot{*}_{r}}\left[\bar{c}_{v}\left(X_{s}\right)-\Lambda_{\mathrm{m}}^{*}-\alpha\right] \mathrm{d} s} \Phi_{\alpha}\left(X_{\breve{\tau}_{r}}\right)\right]+\zeta_{\alpha} \mathbb{E}_{x}^{v}\left[\int_{0}^{\breve{\tau}_{r}} \mathrm{e}^{\int_{0}^{t}\left[\bar{c}_{v}\left(X_{s}\right)-\Lambda_{\mathrm{m}}^{*}-\alpha\right] \mathrm{d} s} \mathrm{~d} t\right]
$$

for all $x \in B_{r}^{c}, r>0$, and $v \in \mathfrak{U}_{\mathrm{SM}}^{*}$.

We next show that $\inf _{\mathbb{R}^{d}} \Phi_{\alpha} \geq M$ for some constant $M>0$ and all $\alpha>0$ sufficiently small. Let $\theta>0$ be such that $c$ is near monotone relative to $\Lambda(f)+2 \theta$, and let $\mathcal{K}_{\theta}$ be as in in Definition 1.1. If $\hat{v} \in \mathfrak{U}_{\mathrm{SM}}$ is a selector from the minimizer of (3.25), then by Itô's formula and Fatou's lemma we obtain

$$
\Phi_{\alpha}(x) \geq \zeta_{\alpha} \mathbb{E}_{x}^{\hat{v}}\left[\int_{0}^{\infty} \mathrm{e}^{\int_{0}^{t}\left[\bar{c}_{\hat{v}}\left(X_{s}\right)-\Lambda_{\mathrm{m}}^{*}-\alpha\right] \mathrm{d} s} \mathrm{~d} t\right] \geq \frac{\zeta_{\alpha}}{\Lambda_{\mathrm{m}}^{*}+\alpha} \quad \forall x \in \mathbb{R}^{d},
$$

since the running cost $c$ is nonnegative. From now on, we select $\zeta_{\alpha}$ so that $\Phi_{\alpha}(0)=1$. Thus $\zeta_{\alpha} \leq \Lambda_{\mathrm{m}}^{*}+\alpha$ by (3.28). Since $\zeta_{\alpha}$ is bounded, the pde in (3.25) satisfies the assumptions for the Harnack inequality for a class of superharmonic functions in [1]. This implies that if $\mathcal{B}$ is some fixed ball containing $\mathcal{K}_{\theta}$, then

$$
C_{H}^{-1} \leq \Phi_{\alpha}(x) \leq C_{H}, \quad \forall x \in \overline{\mathcal{B}}, \quad \forall \alpha \in(0,1),
$$

for some constant $C_{H}$. We leave it to the reader to verify that the assertions in Lemma 2.1 are true for any supersolution of (1.15). Therefore, since $\inf _{\mathbb{R}^{d}} \Phi_{\alpha}>0$, it follows from (3.25) that $\inf _{\mathbb{R}^{d}} \Phi_{\alpha}=\inf _{\mathcal{K}} \Phi_{\alpha} \geq C_{H}^{-1}$ for all $\alpha \in(0, \theta)$.

On the other hand, as in (2.23), for any $v \in \mathfrak{U}_{\mathrm{SM}}^{*}$, we obtain

$$
\mathbb{E}_{x}^{v}\left[\int_{0}^{\breve{\tau}} \mathrm{e}^{\int_{0}^{t}\left[\bar{c}_{v}\left(X_{s}\right)-\Lambda_{\mathrm{m}}^{*}\right] \mathrm{d} s} \mathrm{~d} t\right] \leq\left(\theta \inf _{\partial \mathcal{B}} V_{v}\right)^{-1} V_{v}(x) \quad \forall x \in \mathcal{B}^{c},
$$


where $\mathcal{B}$ is the ball in the preceding paragraph, and $V_{v}$ is as in (3.24). It then follows by (3.27), (3.29), and (3.30) that the collection $\left\{\Phi_{\alpha}, \alpha \in(0,1)\right\}$ is locally bounded, and thus also relatively weakly compact in $\mathcal{W}^{2, p}\left(B_{R}\right)$ for any $p \geq 1$ and $R>0$, by (2.20). Thus passing to the limit in (3.25) as $\alpha \searrow 0$ along some sequence, we obtain a positive $\bar{V} \in \mathcal{C}^{2}\left(\mathbb{R}^{d}\right)$, and a constant $\bar{\zeta}$ solving

$$
\min _{u \in \mathbb{U}}\left[\mathcal{L}^{u} \bar{V}(x)+\left(c(x, u)-\Lambda_{\mathrm{m}}^{*}\right) \bar{V}(x)\right]=-\bar{\zeta} \quad \forall x \in \mathbb{R}^{d} .
$$

Then $\inf _{\mathbb{R}^{d}} \bar{V} \geq C_{H}^{-1}$. Thus, the assumption that $\Lambda_{\mathrm{m}}^{*}(c+h)>\Lambda_{\mathrm{m}}^{*}(c)$ for all $h \in \mathcal{C}_{\mathrm{o}}$, then implies that $\bar{\zeta}=0$. It follows by (3.27)-(3.30), that

$$
\bar{V}(x) \leq \mathbb{E}_{x}^{v}\left[\mathrm{e}^{\int_{0}^{\breve{r}}\left[\bar{c}_{v}\left(X_{s}\right)-\Lambda_{\mathrm{m}}^{*}\right] \mathrm{d} s} \bar{V}\left(X_{\breve{\tau}}\right)\right] \quad \forall x \in \mathcal{B}^{c}, \quad \forall v \in \mathfrak{U}_{\mathrm{SM}}^{*} .
$$

The rest follows exactly as in the proof of Theorem 1.2 .

Remark 3.2. The reader might have noticed not only the different approach in the proof of the existence results in Propositions 1.1 and 1.3. but also the difference between the method of proof of Theorem 1.2 and that of the analogous statement in Proposition 1.3. This needs some explanation. Consider the following definitions of eigenvalues.

$$
\begin{aligned}
& \hat{\Lambda}^{*}:=\inf \left\{\lambda \in \mathbb{R}: \exists \varphi \in \mathcal{W}_{\text {loc }}^{2, d}\left(\mathbb{R}^{d}\right), \varphi>0, \min _{u \in \mathbb{U}}\left[\mathcal{L}^{u} \varphi+(c(x, u)-\lambda) \varphi\right] \leq 0 \text { a.e. in } \mathbb{R}^{d}\right\}, \\
& \hat{\Lambda}_{\mathrm{m}}^{*}:=\inf _{v \in \mathfrak{U}_{\mathrm{SM}}} \inf \left\{\lambda \in \mathbb{R}: \exists \varphi \in \mathcal{W}_{\text {loc }}^{2, d}\left(\mathbb{R}^{d}\right), \varphi>0, \mathcal{L}_{v} \varphi+\left(\bar{c}_{v}-\lambda\right) \varphi \leq 0 \text { a.e. in } \mathbb{R}^{d}\right\} .
\end{aligned}
$$

Also define $\hat{\hat{\Lambda}}^{*}$ and $\hat{\Lambda}_{\mathrm{m}}^{*}$ in direct analogy to these but with $\varphi>0$ in the qualifier replaced by $\inf _{\mathbb{R}^{d}} \varphi>0$. It is straightforward to verify that $\hat{\Lambda}^{*}=\hat{\Lambda}_{\mathrm{m}}^{*} \leq \hat{\Lambda}_{\mathrm{m}}^{*}$. On the other hand since $c$ is near monotone relative to $\Lambda^{*}$, then by Lemma 2.1 and the proof of Theorem 1.4 we have $\hat{\Lambda}^{*}=\hat{\Lambda}_{\mathrm{m}}^{*}=\Lambda_{\mathrm{m}}^{*}$, so we obtain

$$
\hat{\Lambda}^{*}=\hat{\Lambda}_{\mathrm{m}}^{*} \leq \Lambda^{*} \leq \hat{\Lambda}^{*}=\hat{\Lambda}_{\mathrm{m}}^{*}=\Lambda_{\mathrm{m}}^{*} .
$$

Recall from the proof of Theorem 3.4 that $\lambda^{*}$ denotes the limit, as $n \rightarrow \infty$, of the Dirichlet eigenvalues $\hat{\lambda}_{n}$ defined in Lemma 3.1. It is evident that $\lambda^{*} \leq \hat{\Lambda}^{*}$, and since $\lambda^{*}$ satisfies (3.6), we have in fact equality. We have also shown that $\lambda^{*}=\Lambda^{*}$. Note then that under Assumption 1.1 we have $\hat{\Lambda}_{\mathrm{m}}^{*}=\hat{\Lambda}_{\mathrm{m}}^{*}$, so that all the quantities in (3.31) are equal.

However, in the absence of Assumption 1.1 we might have $\hat{\Lambda}_{\mathrm{m}}^{*}<\hat{\Lambda}_{\mathrm{m}}^{*}$, which implies $\lambda^{*}<\hat{\Lambda}_{\mathrm{m}}^{*}$, so a limit point of the Dirichlet eigensolutions will not in general yield a solution to (3.22) under the assumptions of Proposition 1.3

The situation with the proof of Theorem 1.2 is more subtle. If, in the absence of Assumption 1.1, we consider the Dirichlet problems in (3.13) but with $\Lambda^{*}$ replaced by $\Lambda_{\mathrm{m}}^{*}$, then we indeed obtain a positive solution $\Phi$ of (3.14), but we cannot argue that $\Phi$ is inf-compact. Therefore, we cannot use the method in the proof of Lemma 2.11 to conclude that the assumption $\Lambda_{\mathrm{m}}^{*}(c+h)>\Lambda_{\mathrm{m}}^{*}(c)$ for all $h \in \mathcal{C}_{\circ}$ implies $\alpha=0$ in (3.14), and the argument breaks down. Instead, we use a more elaborate method for the proof of the analogous statement in Proposition 1.3 .

\section{Acknowledgement}

We are indebted to the anonymous referee for his helpful comments, and especially for discovering an error in the first version of this paper. His/her review helped us to improve the paper substantially.

The research of Ari Arapostathis was supported in part by the Office of Naval Research through grant N00014-14-1-0196, and in part by the Army Research Office through grant W911NF-17-1-001. The research of Anup Biswas was supported in part by INSPIRE faculty fellowship No. IFA13/MA-32. 


\section{References}

[1] A. Arapostathis, M. K. Ghosh, and S. I. Marcus. Harnack's inequality for cooperative weakly coupled elliptic systems. Comm. Partial Differential Equations, 24(9-10):1555-1571, 1999.

[2] A. Arapostathis, V. S. Borkar, and M. K. Ghosh. Ergodic control of diffusion processes, volume 143 of Encyclopedia of Mathematics and its Applications. Cambridge University Press, Cambridge, 2011.

[3] S. Balaji and S. P. Meyn. Multiplicative ergodicity and large deviations for an irreducible Markov chain. Stochastic Process. Appl., 90(1):123-144, 2000.

[4] A. Bensoussan and R. J. Elliott. A finite-dimensional risk-sensitive control problem. SIAM J. Control Optim., 33(6): 1834-1846, 1995.

[5] A. Bensoussan and H. Nagai. Conditions for no breakdown and Bellman equations of risk-sensitive control. Appl. Math. Optim., 42(2):91-101, 2000.

[6] A. Bensoussan, J. Frehse, and H. Nagai. Some results on risk-sensitive control with full observation. Appl. Math. Optim., $37(1): 1-41,1998$.

[7] H. Berestycki and L. Rossi. Generalizations and properties of the principal eigenvalue of elliptic operators in unbounded domains. Comm. Pure Appl. Math., 68(6):1014-1065, 2015.

[8] H. Berestycki, L. Nirenberg, and S. R. S. Varadhan. The principal eigenvalue and maximum principle for second-order elliptic operators in general domains. Comm. Pure Appl. Math., 47(1):47-92, 1994.

[9] T. R. Bielecki and S. R. Pliska. Risk-sensitive dynamic asset management. Appl. Math. Optim., 39(3):337-360, 1999.

[10] A. Biswas. Risk sensitive control of diffusions with small running cost. Appl. Math. Optim., 64(1):1-12, 2011.

[11] A. Biswas. An eigenvalue approach to the risk sensitive control problem in near monotone case. Systems Control Lett., 60(3):181-184, 2011.

[12] A. Biswas, V. S. Borkar, and K. Suresh Kumar. Risk-sensitive control with near monotone cost. Appl. Math. Optim., 62 (2):145-163, 2010 .

[13] V. I. Bogachev, N. V. Krylov, and M. Röckner. On regularity of transition probabilities and invariant measures of singular diffusions under minimal conditions. Comm. Partial Differential Equations, 26(11-12):2037-2080, 2001.

[14] V. S. Borkar and K. S. Kumar. Small noise large time asymptotics for the normalized Feynman-Kac semigroup. In Variational and optimal control problems on unbounded domains, volume 619 of Contemp. Math., pages 31-48. Amer. Math. Soc., Providence, RI, 2014.

[15] V. S. Borkar and S. P. Meyn. Risk-sensitive optimal control for Markov decision processes with monotone cost. Math. Oper. Res., 27(1):192-209, 2002.

[16] R. Cavazos-Cadena. Optimality equations and inequalities in a class of risk-sensitive average cost Markov decision chains. Math. Methods Oper. Res., 71(1):47-84, 2010.

[17] R. Cavazos-Cadena and D. Hernández-Hernández. A characterization of the optimal risk-sensitive average cost in finite controlled Markov chains. Ann. Appl. Probab., 15(1A):175-212, 2005.

[18] R. Cavazos-Cadena and D. Hernández-Hernández. Necessary and sufficient conditions for a solution to the risk-sensitive Poisson equation on a finite state space. Systems Control Lett., 58(4):254-258, 2009.

[19] Y.-Z. Chen and L.-C. Wu. Second order elliptic equations and elliptic systems, volume 174 of Translations of Mathematical Monographs. American Mathematical Society, Providence, RI, 1998.

[20] G. B. Di Masi and L. Stettner. Risk-sensitive control of discrete-time Markov processes with infinite horizon. SIAM J. Control Optim., 38(1):61-78 (electronic), 1999.

[21] G. B. Di Masi and E. Stettner. Risk-sensitive control of an ergodic diffusion over an infinite horizon. In Proceedings of the Seminar on Stability Problems for Stochastic Models, Part I (Nalęczow, 1999), volume 105, pages 2541-2549, 2001.

[22] G. B. Di Masi and E. Stettner. Infinite horizon risk sensitive control of discrete time Markov processes under minorization property. SIAM J. Control Optim., 46(1):231-252 (electronic), 2007.

[23] D. Down, S. P. Meyn, and R. L. Tweedie. Exponential and uniform ergodicity of Markov processes. Ann. Probab., 23(4): 1671-1691, 1995.

[24] W. H. Fleming. Some results and problems in risk sensitive stochastic control. Mat. Apl. Comput., 16(2):99-115, 1997.

[25] W. H. Fleming. Risk sensitive stochastic control and differential games. Commun. Inf. Syst., 6(3):161-177, 2006.

[26] W. H. Fleming and W. M. McEneaney. Risk-sensitive control on an infinite time horizon. SIAM J. Control Optim., 33 (6):1881-1915, 1995.

[27] W. H. Fleming and S. J. Sheu. Risk-sensitive control and an optimal investment model. II. Ann. Appl. Probab., 12(2): 730-767, 2002.

[28] G. Fort and G. O. Roberts. Subgeometric ergodicity of strong Markov processes. Ann. Appl. Probab., 15(2):1565-1589, 2005.

[29] D. Gilbarg and N. S. Trudinger. Elliptic partial differential equations of second order, volume 224 of Grundlehren der Mathematischen Wissenschaften. Springer-Verlag, Berlin, second edition, 1983.

[30] I. Gyöngy and N. Krylov. Existence of strong solutions for Itô's stochastic equations via approximations. Probab. Theory Related Fields, 105(2):143-158, 1996.

[31] R. Z. Has'minskii. Stochastic stability of differential equations. Sijthoff \& Noordhoff, The Netherlands, 1980.

[32] R. A. Howard and J. E. Matheson. Risk-sensitive Markov decision processes. Management Sci., 18:356-369, 1971/72.

[33] N. Ichihara. Large time asymptotic problems for optimal stochastic control with superlinear cost. Stochastic Process. Appl., 122(4):1248-1275, 2012.

[34] A. Jaśkiewicz. Average optimality for risk-sensitive control with general state space. Ann. Appl. Probab., 17(2):654-675, 2007. 
[35] H. Kaise and S.-J. Sheu. On the structure of solutions of ergodic type Bellman equation related to risk-sensitive control. Ann. Probab., 34(1):284-320, 2006.

[36] I. Kontoyiannis and S. P. Meyn. Spectral theory and limit theorems for geometrically ergodic Markov processes. Ann. Appl. Probab., 13(1):304-362, 2003.

[37] I. Kontoyiannis and S. P. Meyn. Large deviations asymptotics and the spectral theory of multiplicatively regular Markov processes. Electron. J. Probab., 10:no. 3, 61-123 (electronic), 2005.

[38] N. V. Krylov. Controlled diffusion processes, volume 14 of Applications of Mathematics. Springer-Verlag, New York, 1980.

[39] N. V. Krylov and M. Röckner. Strong solutions of stochastic equations with singular time dependent drift. Probab. Theory Related Fields, 131(2):154-196, 2005

[40] R. S. Liptser and A. N. Shiryayev. Statistics of random processes. I. Springer-Verlag, New York, 1977. General theory, Translated by A. B. Aries, Applications of Mathematics, Vol. 5.

[41] J.-L. Menaldi and M. Robin. Remarks on risk-sensitive control problems. Appl. Math. Optim., 52(3):297-310, 2005.

[42] H. Nagai. Bellman equations of risk-sensitive control. SIAM J. Control Optim., 34(1):74-101, 1996.

[43] H. Nagai. Downside risk minimization via a large deviations approach. Ann. Appl. Probab., 22(2):608-669, 2012.

[44] A. Nagengast, D. Braun, and D. Wolpert. Risk-sensitive optimal feedback control accounts for sensorimotor behavior under uncertainty. PLoS Computational Biology, 6(7):e1000857, 2010.

[45] R. G. Pinsky. Positive harmonic functions and diffusion, volume 45 of Cambridge Studies in Advanced Mathematics. Cambridge University Press, Cambridge, 1995.

[46] A. Quaas and B. Sirakov. Principal eigenvalues and the Dirichlet problem for fully nonlinear elliptic operators. Adv. Math., 218(1):105-135, 2008.

[47] J. L. Speyer. An adaptive terminal guidance scheme based on an exponential cost criterion with application to homing missile guidance. IEEE Trans. Automatic Control, 21(3):371-375, 1976.

[48] K. Suresh Kumar and C. Pal. Risk-sensitive ergodic control of continuous time Markov processes with denumerable state space. Stoch. Anal. Appl., 33(5):863-881, 2015.

[49] P. Whittle. Risk-sensitive linear/quadratic/Gaussian control. Adv. in Appl. Probab., 13(4):764-777, 1981.

[50] P. Whittle. Risk-sensitive optimal control. Wiley-Interscience Series in Systems and Optimization. John Wiley \& Sons, Ltd., Chichester, 1990.

[51] L. M. Wu. Feynman-Kac semigroups, ground state diffusions, and large deviations. J. Funct. Anal., 123(1):202-231, 1994.

[52] Y. Yoshimura. A note on demi-eigenvalues for uniformly elliptic Isaacs operators. Viscosity Solution Theory of Differential Equations and its Developments, pages 106-114, 2006. 